\title{
Mean-Field Games and Ambiguity Aversion
}

by

\author{
Xuancheng Huang
}

A thesis submitted in conformity with the requirements

for the degree of Doctor of Philosophy

Graduate Department of Statistical Sciences

University of Toronto

(c) Copyright 2017 by Xuancheng Huang 


\author{
Abstract \\ Mean-Field Games and Ambiguity Aversion \\ Xuancheng Huang \\ Doctor of Philosophy \\ Graduate Department of Statistical Sciences \\ University of Toronto
}

2017

This thesis focuses on incorporating the idea of ambiguity aversion into mean-field games. Intuitively, mean-field games describes the dynamics of a system with an infinite population. It is useful in approximating systems with a large population, for which an exact result is computationally intractable. By adding in ambiguity aversion, the mean-field game reflects how players in the population should act if they wish to protect themselves from model misspecification.

Two applications of mean-field games are considered through two distinct approaches. The broker execution problem is investigated in a multi-agent framework containing (i) a major agent who is liquidating a large number of shares, (ii) a number of minor agents (high-frequency traders (HFTs)) who search for statistical arbitrage strategies, and (iii) noise traders who buy and sell for exogenous reasons. All optimizing agents (the broker and HFTs) trade against noise traders as well as one another. We use a mean-field game approach to solve the problem and obtain a set of decentralized feedback trading strategies for the major and minor agents. Furthermore, the mean-field game strategies have an $\epsilon_{N}$-Nash equilibrium property where $\epsilon_{N} \rightarrow 0$ as $N \rightarrow \infty$.

The second application focuses on interbank borrowing and lending, which may induce systemic risk into financial markets. A simple model of this is to assume that log-monetary reserves are coupled, and that banks can also borrow/lend from/to a central bank. When all banks optimize their cost of borrowing and lending, this leads to a stochastic game. We account for model uncertainty by recasting the problem as a robust stochastic game and succeed in providing a strategy which leads to a Nash equilibria for 
both the finite game and the mean-field game limit. We prove that an $\epsilon$-Nash equilibrium exists and show that when firms are ambiguity-averse, default probabilities can be reduced relative to their ambiguity-neutral counterparts.

Finally, this thesis develops a modified stochastic maximum principle for min-max problems, and derives new existence and uniqueness results for a mean-field game with ambiguity averse players. An $\epsilon$-Nash equilibrium is shown to exist for this class of mean-field games, and the mean-field game equations are explicitly derived in the linearquadratic framework. 


\section{Acknowledgements}

There are many people whom I wish to thank for their support throughout my PhD:

- My supervisor Sebastian Jaimungal. You've given me an enormous amount of guidance and support throughout the past four years. This thesis would not have been possible without your insights and encouragement.

- My committee members Dan Roy, Jeffrey Rosenthal and Sheldon Lin for providing their perspectives and feedback on my work

- The staff: Annette Courtmanche, Andrea Carter, Christine Bulguryemez, Gillis Aning and Angela Fleury. You have helped me with everything from paperwork to organizing the Research Day conference.

- Professors Jamie Stafford, Radu Craiu, Nancy Reid and Andrey Feuerverger. You have always taken an interest in my development, and been willing to offer advice during my time in the department.

- Professors Sam Broverman and Vicki Zhang. You encouraged me to take on my first teaching position, and helped me develop my teaching abilities.

- My parents Xianhua Huang and Tingwei Lu, who have always encouraged and nurtured my curiosity during my studies. Your continual support has always been a pillar on which I could lean on in difficult times. None of this would have been possible without you.

- My girlfriend Yi Serena Lu. For always being there for me. You were always willing to listen to me ramble on about my research, even when you just wanted to go for a peaceful walk outside.

- My friends at the University of Toronto, in particular: Karen Huynh Wong, Wei Deng, Victor Veitch, Luhui Gan, Tianyi Jia, Philippe Casgrain, Ali Al-Aradi, Zhenhua Lin, Alex Shestopaloff, and David Farahany. For lending an ear and letting me bounce ideas off of you.

- The Government of Ontario, for providing funding through the Queen Elizabeth II Graduate Scholarships in Science and Technology. 


\section{Contents}

1 Introduction $\quad 1$

1.1 Mean Field Games . . . . . . . . . . . . . . . . . . . . . . . . . 1

1.1.1 Pontryagin Maximum Principle . . . . . . . . . . . . . 3

1.1.2 Hamilton-Jacobi-Bellman Equation . . . . . . . . . . . . . . 3

1.1.3 The Master Equation . . . . . . . . . . . . . . . . . . 4

1.2 Solving the Mean-Field Game . . . . . . . . . . . . . . . . 5

1.2.1 Mean-Field Games with Major and Minor Agents . . . . . . . . 5

1.2.2 Variational Preferences and Ambiguity Aversion . . . . . . . . 6

1.3 Outline and Contributions . . . . . . . . . . . . . . . . 9

2 Mean-Field Games and Optimal Execution $\quad 12$

2.1 Introduction . . . . . . . . . . . . . . . . . . . . . . . 12

2.2 Major-Minor Agent Optimal Execution Problem . . . . . . . . . . . . 15

2.2.1 Trading Dynamics of Major and Minor Agents . . . . . . . . 16

2.2 .2 Noise Traders . . . . . . . . . . . . . . . . . . . . 17

2.2.3 Fundamental Price Dynamics . . . . . . . . . . . . . . . 18

2.2.4 The Agents' Performance Criteria . . . . . . . . . . . . . . . . 19

2.3 A mean-field Game Approach . . . . . . . . . . . . . . . . . 20

2.3.1 Infinite Number of Minor Agents . . . . . . . . . . . . . . 21

2.3.2 Solutions to Limiting Stochastic Optimal Control Problems for Major and Minor Agents . . . . . . . . . . . . . 23

2.3 .3 Verification . . . . . . . . . . . . . . 25

2.4 Fixed Point Equations . . . . . . . . . . . . . . . . . . . . 26

2.4.1 Mean-Field Game Fixed Point . . . . . . . . . . . . . . . 26

2.4.2 Behaviour of the mean-field Game System in the Infinite Penalty Set-up . . . . . . . . . . . . . . . 27

2.5 Decentralized mean-field Game Trading Strategies: The $\epsilon_{N}$-Nash Equilibrium Property . . . . . . . . . . . . . . . . . 28 
2.6 Simulations . . . . . . . . . . . . . . . . . . . . . . . . . . . 32

2.6.1 Major and minor agents with same liquidation horizon . . . . . 32

2.6.2 Minor agents with small terminal penalty . . . . . . . . . . 36

2.6.3 Impact of Execution Quantity on Performance . . . . . . . . 37

2.7 Concluding Remarks . . . . . . . . . . . . . . . . . . . . 38

$\begin{array}{lr}\text { Appendices } & 40\end{array}$

2.A Proof of ODE Derivations . . . . . . . . . . . . . . . . 40

2.A.1 Major Agent ODEs . . . . . . . . . . . . . . . . . . . 40

2.A.2 Minor Agent ODEs . . . . . . . . . . . . . . . . 42

2.B Proof of $\epsilon$-Nash Equilibrium . . . . . . . . . . . . . . . . . 44

2.B.1 Proof of $2.5 .1 \ldots \ldots \ldots \ldots \ldots \ldots$

2.B.2 Proof of $2.40 \ldots \ldots \ldots \ldots \ldots \ldots \ldots \ldots$

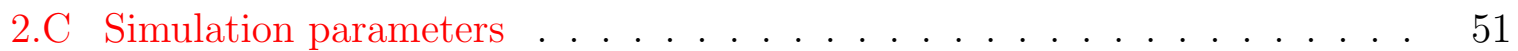

3 Robust Stochastic Games and Systemic Risk 52

3.0 .1 Systemic Risk . . . . . . . . . . . . . . . . . 52

3.1 Introduction . . . . . . . . . . . . . . . . 53

3.2 The Model Setup . . . . . . . . . . . . . . . . . . . . . . . . 54

3.2 .1 Reserve Dynamics . . . . . . . . . . . . . . . . . 56

3.2 .2 Performance Criteria . . . . . . . . . . . . . . . 56

3.3 N Player Game . . . . . . . . . . . . . . . . . . . . . . . . 57

3.3.1 Ambiguity Averse Agents . . . . . . . . . . . . . . . 58

3.3 .2 Closed-Loop Game . . . . . . . . . . . . . . . . . . . 58

3.3.3 FBSDE for closed-loop equilibria . . . . . . . . . . . 60

3.3 .4 Open-Loop Game . . . . . . . . . . . . . . . . . . . . 60

3.4 Mean-field Game . . . . . . . . . . . . . . . . . . . . . . . . 61

3.4 .1 The Mean-Field . . . . . . . . . . . . . . . . . . . 61

3.4 .2 Setup ............................. 61

3.5 Verification Theorem and $\epsilon$-Nash Equilibrium . . . . . . . . . . . 64

3.5.1 $\epsilon$-Nash Equilibrium . . . . . . . . . . . . . . 66

3.6 Default Probability . . . . . . . . . . . . . . . . . . . 67

3.6 .1 PDE Approach . . . . . . . . . . . . . . . 67

3.6 .2 Simulations . . . . . . . . . . . . . . . . . 68

3.6 .3 Financial Implications . . . . . . . . . . . . . . . . . 69

3.7 Concluding Remarks . . . . . . . . . . . . . . . . . . . 70 
$\begin{array}{ll}\text { Appendices } & 71\end{array}$

3.A FBSDE for closed form solution . . . . . . . . . . . . . . . 71

3.B Mean-Field Game Optimal Control . . . . . . . . . . . . . . . . 72

3.C Verification Theorem ... . . . . . . . . . . . . . . 74

3.D Default. . . . . . . . . . . . . . . . . . 76

3.D.1 Comparison for PDE . . . . . . . . . . . . . . 80

4 Mean-Field Games and Ambiguity Aversion $\quad 82$

4.1 Introduction . . . . . . . . . . . . . . . . . . . . 82

$4.1 .1 \quad$ N-player game . . . . . . . . . . . . . . . . . . . 83

4.1 .2 Mean-Field Game . . . . . . . . . . . . . . . . . . . . . 83

4.1 .3 Optimizing via the Hamiltonian . . . . . . . . . . . . . . . 84

4.2 Stochastic Nash-Pontryagin Principle . . . . . . . . . . . . . . . 86

4.3 Mean-Field FBSDE . . . . . . . . . . . . . . . . . . . . . . . . . . . . . . 91

$4.4 \quad \epsilon$-Nash equilibrium . . . . . . . . . . . . . . . . . . . . . . . . 94

4.4 .1 Comments on assumptions . . . . . . . . . . . . . 98

4.5 Linear-Quadratic Game . . . . . . . . . . . . . . . . . . . . . . . . . 99

4.5.1 Problem Formulation . . . . . . . . . . . . . . . . . . . 99

$4.5 .2 \epsilon$-Nash equilibrium . . . . . . . . . . . . . . . . . 101

4.5 .3 Comments . . . . . . . . . . . . . . . . . . . 101

5 Background 102

5.1 Probability . . . . . . . . . . . . . . . . . . . . . . 102

5.2 Hamilton-Jacobi-Bellman Principle . . . . . . . . . . . . . . . . . 102

5.3 Pontryagin Stochastic Minimum Principle . . . . . . . . . . . . . 103

5.4 Ansatz . . . . . . . . . . . . . . . . . . . . . . 103

5.5 Almgren-Criss Framework . . . . . . . . . . . . . . . . 103

6 Future work 106

6.1 Optimal Execution . . . . . . . . . . . . . . . . . . . 106

6.2 Systemic Risk . . . . . . . . . . . . . . . . . . . . . . . . 107

6.3 Mean-field Games with Ambiguity Aversion . . . . . . . . . . . . . 108 


\section{Chapter 1}

\section{Introduction}

Game theory is an essential tool in understanding interactions in financial markets. Although there are numerous mathematical finance models suitable for pricing and trading small quantities of derivatives, large-scale movements in assets can cause behaviours which cannot be predicted with these models. Game theory is one way of describing how other players in financial markets react to information.

Classical game theory is a well developed field, but the computational tractability decreases significantly as the number of players increases. Mean-field games is an approach for solving an approximate version of such games when $N$, the number of players, is very large. In classical game theory, each player has a strategy which satisfies a Nash equilibrium, so that no player has any incentive to deviate from their optimal strategy. In mean-field game theory, each player's optimal strategy satisfies an $\epsilon$-Nash equilibrium instead, so that a player can only gain $\epsilon$ by deviating from the mean-field game strategy, and $\epsilon \rightarrow 0$ as $N \rightarrow \infty$. The main issues to address in mean-field game theory are (i) does a mean-field equilibrium exist, (ii) is the equilibrium unique and (iii) does the mean-field game strategy satisfy an $\epsilon$-Nash equilibrium property. From a practical perspective, there is also the question of whether the dynamics of the players in the mean-field game can be solved for explicitly, so that the optimal control has a closed-form or numerical solution.

This chapter will cover some common approaches to solving mean-field games, as well an overview of incorporating ambiguity aversion into continuous time models.

\subsection{Mean Field Games}

Throughout the thesis, we will be working primarily with diffusion processes. We first look at techniques for non-game theoretic problems. In stochastic control theory, the set 
of admissible controls are restricted to be square integrable as follows

$$
\mathcal{A}=\left\{\alpha_{t} \mid \mathbb{E}\left[\alpha_{t}^{2}\right]<\infty\right\}
$$

Let $W_{t}$ be a standard Brownian motion with filtration $\left\{\mathcal{F}_{t}\right\}, b:[0, T] \times \mathbb{R} \times \mathcal{A} \rightarrow \mathbb{R}$ and $\sigma^{i}:[0, T] \rightarrow \mathbb{R}$ denote the drift and volatility of the process $X_{t}$, and $\left\{\alpha_{t}\right\}_{0, T}$ be a control within the set of admissible controls $\mathcal{A}$. The dynamics of the agent is given by the SDE

$$
d X_{t}=b\left(t, X_{t}, \alpha_{t}\right) d t+\sigma_{t} d W_{t}
$$

and cost function that the player wishes to minimize is

$$
J\left(\alpha_{t}\right)=\mathbb{E}\left[\int_{0}^{T} f\left(t, X_{t}, \alpha_{t}\right) d t+g\left(T, x_{T}\right)\right]
$$

This is a standard continuous-time optimization problem and can be solved (under certain assumptions) with the Hamilton-Jacobi-Bellman principle as in Øksendal and Sulem (2009) or the stochastic maximum principle as in Peng (1990).

Let us assume that there are $\mathrm{N}$ players in a game, and each player has an action she can take denoted $\alpha^{i} \in \mathbb{R}^{d}$. Then, by defining the cost function for player $i$ as $J^{i}\left(\alpha_{t}^{i}, \alpha_{t}^{-i}\right)$, where $\alpha_{t}^{-i}=\left(\alpha_{t}^{1}, \ldots, \alpha_{t}^{i-1}, \alpha_{t}^{i+1}, \ldots, \alpha_{t}^{N}\right)$, the continuous-time game can be described as a set of $\mathrm{N}$ simultaneous optimization problems. In order to establish a Nash equilibrium, we would have to find a fixed point $\widehat{\boldsymbol{\alpha}}_{t}=\left(\widehat{\alpha}_{t}^{1}, \ldots, \widehat{\alpha}_{t}^{N}\right)$, such that

$$
\widehat{\alpha}_{t}^{i}=\underset{\alpha_{t}^{i}}{\arg \inf } J^{i}\left(\alpha_{t}^{i} ; \widehat{\alpha}_{t}^{-i}\right) \quad i=1, \ldots, N
$$

In general, such a fixed point would be difficult to find, given the growth in dimension of the search space. However, by defining the limiting distribution of the fixed point

$$
\mu_{t}=\lim _{N \rightarrow \infty} \frac{1}{N} \sum_{i=1}^{N} \delta_{X_{t}^{i}}
$$

then the set of simultaneous optimization problems can be reduced to independent continuous-time optimization problems for each player. Such a solution generally requires the structure of the problem to be exchangeable in the dynamics and cost functions of the players. 


\subsubsection{Pontryagin Maximum Principle}

The Pontryagin Maximum Principle is useful for convex problems and relies on the concept of a Hamiltonian.

Definition 1.1.1. Given the dynamics (1.2) and cost function (1.3), the Hamiltonian is defined as

$$
\begin{aligned}
H(t, x, \mu, \alpha, y) & =\langle b(t, x, \mu, \alpha), y\rangle+f(t, x, \alpha) \\
H\left(T, X_{T}, \mu_{T}, \cdot, \cdot\right) & =g\left(X_{T}, \mu_{T}\right)
\end{aligned}
$$

Using the version of the Pontryagin Maximum Principle for mean-field games, Carmona et al. (2015), Carmona and Delarue (2013) show that the optimal controls to the mean-field game version of the problem is the solution to a forward-backwards stochastic differential equation (FBSDE).

$$
\begin{aligned}
d X_{t} & =\partial_{y} H\left(t, X_{t}, \mu_{t}, \widehat{\alpha}_{t}, Y_{t}, Z_{t}\right) d t+\sigma d W_{t} \\
d Y_{t} & =-\partial_{x} H\left(t, X_{t}, \mu_{t}, \widehat{\alpha}_{t}, Y_{t}, Z_{t}\right)+Z_{t} d W_{t} \\
X_{0} & =x_{0} \\
Y_{T} & =\partial_{x} g\left(X_{T}, \mu_{T}\right)
\end{aligned}
$$

where

$$
\widehat{\alpha}_{t}=\underset{\alpha_{t}}{\arg \inf } H\left(t, x, \mu, \alpha_{t}, y\right)
$$

For a fixed measure flow, $\left(\mu_{t}\right)_{0 \leq t \leq T}$, and under the assumption of convexity of the cost function, this becomes a convex optimization problem, and the FBSDE is of McKeanVlasov type.

\subsubsection{Hamilton-Jacobi-Bellman Equation}

When applying the Hamilton-Jacobi-Bellman (HJB) equation to the problem with a frozen measure flow, the optimization problem becomes

$$
V^{*}(x, t)=\inf _{\alpha_{t}}\left\{\int_{0}^{t} f\left(t, x_{t}, \mu_{t}, \alpha_{t}\right) d t+g\left(x_{T}, \mu_{T}\right)\right\}
$$


and with the optimal control $\widehat{\alpha}, V^{*}$ satisfies the PDE

$$
\begin{aligned}
\partial_{t} V_{t}^{*}+H\left(t, x, \mu_{t}, \widehat{\alpha}, \partial_{x} V_{t}^{*}\right)+\frac{1}{2} \sigma_{t}^{2} x^{2} \partial_{x x}^{2} V_{t}^{*} & =0 \\
V_{T}^{*}(x) & =g\left(x, \mu_{T}\right)
\end{aligned}
$$

This characterizes the optimal control in terms of a 1-dimensional PDE, and is generally a viscosity solution to the PDE. For the Hamilton-Jacobi-Bellman approach, solving a PDE by itself is not sufficient, as the measure flow was a priori assumed to be frozen. In order for the solution to be consistent, a fixed point must be found such that $\operatorname{Law}\left(X_{t}\right)=$ $\mu_{t}$. As a result, a second (forwards) PDE is introduced for the dynamics measure flow $\mu_{t}$ such as in Huang et al. (2006), Lasry and Lions (2007a) and Bauso et al. (2016). The resulting pair of PDEs are as follows:

$$
\begin{aligned}
\partial_{t} V(t, x)+\frac{\sigma^{2}}{2} \Delta_{x} V(t, x)+H\left(t, x, \mu_{t}, \widehat{\alpha}\left(t, x, \mu_{t}, \nabla_{x} V(t, x)\right), \nabla_{x} V(t, x)\right) & =0 \\
\partial_{t} \mu_{t}-\frac{\sigma^{2}}{2} \Delta_{x} \mu_{t}+\operatorname{div}_{x}\left(b\left(t, x, \mu_{t}, \widehat{\alpha}\left(t, x, \mu_{t}, \nabla_{x} V(t, x)\right)\right) \mu_{t}\right) & =0 \\
V(T, x) & =g\left(x, \mu_{T}\right) \\
\mu_{0} & =\delta_{x_{0}}
\end{aligned}
$$

\section{Connection between HJB and Pontryagin Maximum Principle}

Under certain regularity conditions, if both the HJB and Pontryagin Maximum Principle have a solution, then their solutions can be shown to be equivalent in the sense that

$$
Y_{t}=\partial_{x} V_{t}, \quad Z_{t}=\partial_{x x} V_{t}
$$

The conditions and proof are provided in Pham (2009). The main difference between the solutions provided by the two approaches is that the HJB equation gives us a forwardbackwards partial differential equation, while the Pontryagin maximum principle gives us a forward-backwards stochastic differential equation. For a verification theorem to hold, the HJB equation generally requires a quadratic growth condition on the cost function and solution, while the Pontryagin maximum principle requires joint convexity in $(x, \alpha)$ for the cost function.

\subsubsection{The Master Equation}

In the above formulations, the solutions were characterized in terms of a forward equation and a backwards equation. For mean-field games, a more general result holds in the form 
of a master equation, which is a second-order PDE on the space of probability measures. Although this thesis does not discuss the master equation, we nonetheless refer interested readers to Cardaliaguet et al. (2015), Carmona and Delarue (2014), Bensoussan et al. (2015) for details. However, in order to obtain explicit solutions, it is preferable to work with forwards and backwards equations.

\subsection{Solving the Mean-Field Game}

In general, solving the mean-field game can be broken up into 3 main parts

(i) For a frozen measure flow $\left(\mu_{t}\right)_{0 \leq t \leq T}$, solve the optimization problem for a representative agent, and derive an expression for the optimal control $\left(\widehat{\alpha}_{t}\right)_{0 \leq t \leq T}$.

(ii) Find a fixed point, such that $\operatorname{Law}\left(X_{t}\right)=\left(\mu_{t}\right)_{0 \leq t \leq T}$ when players use the optimal control $\left(\widehat{\alpha}_{t}\right)_{0 \leq t \leq T}$.

(iii) Verify that an $\epsilon$-Nash equilibrium exists, to establish that the mean-field limit is the limit of the finite game as $N \rightarrow \infty$.

\subsubsection{Mean-Field Games with Major and Minor Agents}

An approach to the mean-field game framework incorporating both major and minor agents was pioneered by Huang (2010a), and a separate approach to the problem is given in Carmona and Zhu (2016). Other works which have looked at various aspects of games with both major and minor agents are Nourian and Caines (2013), Huang and Nguyen (2016), and Şen and Caines (2014).

The primary way in which a game with a major and minor agent differs from that of a standard mean-field game is that the major agent will have a non-negligible effect on the players. The setup for a major-minor agent game with a single major agent is as follows. Let $W_{t}^{0}, W_{t}$ be standard Brownian motions with filtrations $\left\{\mathcal{F}_{t}^{0}\right\},\left\{\mathcal{F}_{t}\right\}$ respectively. $b^{1}, \sigma^{1} \in \mathbb{R}$ denote the drift and volatility of the process $X_{t}^{1}$ which is $\mathcal{F}_{t}$ measurable, while $b^{0}, \sigma^{0}$ denote the drift and volatility of the process $X_{t}^{0}$ which is $\left\{\mathcal{F}_{t}^{0}\right\}$ measurable. $\left\{\alpha_{t}^{0}\right\}_{0, T},\left\{\alpha_{t}^{1}\right\}_{0, T} \in[0, T] \times \mathbb{R}$ are the controls for the major agent and minor agents respectively. Given dynamics

$$
\begin{aligned}
& d X_{t}^{0}=b^{0}\left(t, X_{t}^{0}, \mu_{t}, \alpha_{t}^{0}\right) d t+\sigma^{0}(t) d W_{t}^{0} \\
& d X_{t}^{1}=b^{1}\left(t, X_{t}^{1}, \mu_{t}, X_{t}^{0}, \alpha_{t}^{1}\right) d t+\sigma^{1}(t) d W_{t}
\end{aligned}
$$


and cost functions

$$
\begin{aligned}
& J^{0}\left(\alpha_{t}^{0} ; \alpha_{t}^{1}\right)=\mathbb{E}\left[\int_{0}^{T} f^{0}\left(t, X_{t}^{0}, \mu_{t}, \alpha_{t}^{0}\right) d t+g^{0}\left(T, X_{T}^{0}, \mu_{t}\right)\right] \\
& J^{1}\left(\alpha_{t}^{1} ; \alpha_{t}^{0}\right)=\mathbb{E}\left[\int_{0}^{T} f^{1}\left(t, X_{t}^{1}, \mu_{t}, X_{t}^{0}, \alpha_{t}^{1}\right) d t+g^{1}\left(T, X_{T}^{1}, \mu_{t}\right)\right]
\end{aligned}
$$

for the major and minor agents respectively, we wish to minimize the cost functions subject to the dynamics of $\left(X_{t}^{0}, X_{t}^{1}\right)$.

As a result, there is an additional component to solving the mean-field game

(i) For a given measure flow $\left(\mu_{t}^{0}\right)_{0 \leq t \leq T}$, solve the optimization problem for the major agent $\left(\widehat{\alpha}_{t}^{0}\right)_{0 \leq t \leq T}$ and a representative minor agent $\left(\widehat{\alpha}_{t}^{1}\right)_{0 \leq t \leq T}$.

(ii) Find a fixed point, such that $\operatorname{Law}\left(X_{t}^{1} \mid \mathcal{F}_{0}\right)=\mu_{t}^{0}$ when $X_{t}^{0}$ is controlled by $\left(\widehat{\alpha}_{t}^{0}\right)_{0 \leq t \leq T}$ and $X_{t}^{i}$ is controlled by $\left(\widehat{\alpha}_{t}^{i}\right)_{0 \leq t \leq T}$.

(iii) Verify that an $\epsilon$-Nash equilibrium exists, to establish that the mean-field limit is the limit of the finite game as $N \rightarrow \infty$.

When a major agent is added to the problem, they are not explicitly included in the

measure flow $\left(\mu_{t}^{0}\right)_{0 \leq t \leq T}$. The measure $\mu_{t}^{0}$ is still reserved for the limiting distribution of minor agents, as there is only a single major agent present. (In theory, there can be any finite number of major agents present, although this could cause the computational complexity of the problem to be infeasible.) The mean-field measure flow must be adapted to the filtration $\mathcal{F}_{0}$ due to the non-negligible impact of the major agent on the dynamics of the minor agents.

\subsubsection{Variational Preferences and Ambiguity Aversion}

When an optimization problem is formulated, the optimizing player sets a performance criteria to be maximized/minimized. In economics and finance, the player is generally assumed to be risk averse (with a concave utility function), as they would prefer to have a certain, but lower expected payoff, than an uncertain, but higher expected payoff. When the probability distribution is given, risk aversion is a well defined and consistent concept. Ambiguity aversion aims to address the case where players do not know the probability distribution of the states/outcomes. In particular, the Ellsberg paradox illustrates the difference between utility theory and ambiguity aversion. 


\section{Multiplier Preferences}

The concept of ambiguity aversion has been looked at mathematically in Epstein (1999), Hansen et al. (2006) and Maccheroni et al. (2006), by treating model uncertainty in a probabilistic manner. Hansen et al. (2006) looked at agents who rank payoff profiles, with respect to a utility function $u$, according to the criteria (referred to as multiplier preferences)

$$
V(\alpha)=\min _{\mathbb{Q} \sim \mathbb{P}} \mathbb{E}[u(\alpha)+\theta \mathcal{H}(\mathbb{Q} \| \mathbb{P})]
$$

where $\mathcal{H}(\mathbb{Q} \| \mathbb{P})$ is the relative entropy or Kullback-Leibler divergence of $\mathbb{Q}$ with respect to $\mathbb{P}$, and $\mathbb{Q} \sim \mathbb{P}$ denotes that the two probability measures are absolutely continuous with respect to each other.

\section{Variational Preferences}

Maccheroni et al. (2006) extend the setup of Hansen et al. (2006) by considering a more general set of preferences, referred to as variational preferences, which can be written as

$$
V(f)=\min _{\mathbb{Q} \sim \mathbb{P}} \mathbb{E}[u(f)+c(\mathbb{Q})]
$$

where $c$ is a convex function of $\mathbb{Q}$.

We define the weighted relative Gini index as in Maccheroni et al. (2006) by

$$
G(\mathbb{P} \| \mathbb{Q})=\int_{s} w(s) \frac{1}{2}\left(\frac{d \mathbb{P}}{d \mathbb{Q}}-1\right) d \mathbb{Q}(s), \quad \text { if } \mathbb{Q} \sim \mathbb{P}
$$

and where $G=\infty$ otherwise, where $w(s)$ is a weight function.

The mean-variance portfolio theory of Markowitz (1952) :

$$
V(f)=\int f d \mathbb{P}-\frac{1}{2 \theta} \operatorname{Var}(f)
$$

is then equivalent to

$$
\min _{\mathbb{Q} \sim \mathbb{P}}\left[\int f d \mathbb{Q}+\theta G(\mathbb{Q} \| \mathbb{P})\right]
$$

The mean-variance preferences of Markowitz (1952) are therefore a special case of the variation representation of preferences in Maccheroni et al. (2006).

The idea of ambiguity aversion in stochastic games (referred to as robust stochastic games) has been explored in Bauso et al. (2016) using the interpretation of attenuation 
noise. In this thesis, we will look at the formulation of Hansen et al. (2006) in two applications of mean-field games, and Maccheroni et al. (2006) in a general ambiguity averse mean-field game framework. 


\subsection{Outline and Contributions}

This thesis explores the behaviour of mean-field games under model misspecification. We look at two applications, providing a semi-closed form and a closed-form solution to the problems, as well as conditions for which a mean-field game exists when players are ambiguity averse.

Trading volume on exchanges have come to be dominated by algorithmic (AT) and high frequency (HFT) traders. Algorithmic traders rely on computer algorithms to automatically make decisions, and HFTs take advantage of the near-instantaneous response times of computers to execute trades at very high speeds. In 2009-2010, HFTs were quoted as accounting for 60-70\% of US trading. One direction in which algorithmic trading has been applied is in the area of optimal execution problems. The goal of an optimal execution problem is to maximize (minimize) the gain (cost) of buying (selling) a certain portfolio of shares. In the optimal execution framework presented in Almgren and Chriss (2001), the authors look at balancing market impact with price uncertainty. Although most of the literature focuses on single agent settings, in pratice, there are numerous hedge funds and banks who employ statistical arbitrage strategies as well. In aggregate, these agents would have a sizeable impact on the market, and can have a significant impact on optimal execution strategies. The standard Almgren-Criss framework is briefly described in section 5.5.

Chapter 2 investigates a mean-field game framework containing (i) a major agent who is liquidating a large number of shares, (ii) a number of minor agents (high-frequency traders (HFTs)) who employ statistical arbitrage strategies, and (iii) noise traders who buy and sell for exogenous reasons. Section 2.3 describes the player dynamics, with the addition of noise traders as in Cartea and Jaimungal (2016), as well as HFTs as minor agents. This setting incorporates permanent price impact from all traders and accounts for the interaction between the major and minor agents. The major agent and the HFTs trade against the noise traders as well as each other, and are coupled in the price and trade dynamics. A mean-field game approach is given in Section 2.4, where we obtain a set of decentralized feedback trading strategies for the major and minor agents, and express the solution explicitly as the solution to a set of coupled Riccatti ODEs through the fixed point described in Section 2.5. Section 2.6 shows that the set of meanfield strategies are shown to satisfy an $\epsilon_{N}$-Nash equilibrium property, where $\epsilon_{N} \rightarrow 0$ as $N \rightarrow \infty$, so that the mean-field game strategies can be used as approximations for the optimal strategies in the finite player game. Finally, Section 2.7 provides some simulation results based on parameters estimated from real-world stock data for stocks with varying 
levels of liquidity.

Another important topic in finance is systemic risk in financial markets. Since the 2008 crisis, a number of papers such as Haldane and May (2011) and Acharya et al. (2017) have been written on the subject of contagion in financial markets, and the role of systemic risk. In mathematical finance, there have been numerous models considered for interbank borrowing and lending, such as in Fouque and Ichiba (2013) and Carmona et al. (2015), where the authors study the probability of default for a bank. The model proposed in Carmona et al. (2015) assumes that log-monetary reserves are coupled, and that banks can also borrow/lend from/to a central bank. When all banks optimize their cost of borrowing and lending, this leads to a stochastic game which, as Carmona et al. (2015) show, induces more stability in the market.

Chapter 3 extends this model by having banks account for model misspecification in their borrowing and lending decisions. The problem formulation as a robust stochastic game is presented in Section 3.2. Section 3.3 provides an strategy for the banks which leads to a Nash equilibria for the finite game, and looks at the difference in the open-loop and closed-loop game. The mean-field game limit is studied in Section 3.4, and compared with the finite player results. In Section 3.5, an $\epsilon$-Nash equilibrium is shown to hold, and a verification theorem is derived for the optimal controls. Moreover, we show that when firms are ambiguity-averse, default probabilities can be further reduced relative to their ambiguity-neutral counterparts in Section 3.6.

In Chapters 2 and 3, mean-field games are used to derive approximations to optimal controls in stochastic games. The theory of mean-field games was initially developed by Lasry and Lions (2007a) and Huang et al. (2006), with many other works such as Carmona and Delarue (2013) and Carmona et al. (2016) generalizing the existence and uniqueness results. Risk aversion and ambiguity aversion have been studied in Tembine et al. (2011),Bauso et al. (2016) and Huang and Huang (2017). In particular, the model in Lasry and Lions (2007a) was extended to incorporate ambiguity aversion in Bauso et al. (2016) using a PDE approach.

Chapter 4 looks at a general framework for mean-field games with ambiguity averse players built on the probabilistic framework described in Carmona and Delarue (2013). In Section 4.1, we present a framework for mean-field games with ambiguity averse players, using a version of the stochastic maximum principle to find the optimal controls of the players. Section 4.2 presents a rigorous analysis of the controls derived in section 4.1, and section 4.3 characterizes the mean-field forward-backwards stochastic differential equation. Section 4.4 provides a rigorous analysis of the relationship between the meanfield game and finite player game, while the optimal control and player dynamics are 
explicitly derived in section 4.5 for the linear-quadratic framework. 


\section{Chapter 2}

\section{Mean-Field Games and Optimal Execution}

\section{$2.1 \quad$ Introduction}

Algorithmic trading refers to any form of quantitative trading strategy which is executed by computer programs that make decisions automatically. An important class of algorithmic trading strategies is high frequency trading which are algorithms that are executed at a very high speed. Automated trading algorithms have grown rapidly and are now applied across electronic trading platforms worldwide. As a result, there has been a considerable amount of research devoted to the modeling and design of efficient trading strategies. In particular, two related but distinct lines of research include optimal execution problems and optimal limit order placements. In optimal execution problems, an agent is required to choose an efficient trading strategy for liquidating/acquiring a portfolio containing a large quantity of a given security in order to maximize her expected wealth (by minimizing cost or maximizing profit). In this problem formulation, there is a tradeoff between trading slowly to reduce market impact, or execution cost, and trading fast to reduce the risk of future uncertainty in prices (see e.g., Almgren and Chriss (2001); Alfonsi et al. (2010); Gatheral et al. (2012); Guéant and Lehalle (2013); Jaimungal and Kinzebulatov (2014); Cartea and Jaimungal (2016) among many others). Optimal limit order placement aims instead to benefit from buying low and selling high and benefit from the bid-ask spread without being adversely selected. The aim is to optimally place bid and ask limit orders to maximize the expected profit and here the agent faces a tradeoff between trading gains and non-execution risks (see e.g., Bayraktar and Ludkovski (2011, 2014); Guéant et al. (2012); Cartea and Jaimungal (2013); Guilbaud 
and Pham (2015); Cartea et al. (2014); Cartea and Jaimungal (2012), among others).

Most of the literature on algorithmic trading has so far focused on the single agent setting. Part of the reason for this focus is that the accounting for multiple agents leads to high dimensional problems and complicated interactions, and typically renders the analysis intractable using standard techniques in stochastic optimal control. There has, however, been some work in addressing multiple agent problems, see e.g., Brunnermeier and Pedersen (2005); Carmona and Yang (2011); Moallemi et al. (2012); Schied and Zhang (2013); Carlin et al. (2007), among others. In particular, Carmona and Yang (2011) develop a closed-loop Nash equilibrium strategy for a model consisting of a distressed agent who needs to liquidate shares and is facing a predator trader with longer time constraints. A similar setting to Carmona and Yang (2011) is investigated in Moallemi et al. (2012) who study an agent who needs to liquidate a large number of shares and is facing an uninformed arbitrageur who hopes to profit from the liquidator's trading by learning from observed price fluctuations. Schied and Zhang (2013) develop an open-loop Nash equilibrium strategy for a finite population of liquidators who are coupled through fundamental price dynamics and aim to maximize their individual mean-variance utility functions using continuous trading.

Large population dynamical multi-agent competitive phenomena occur in various other fields including communication networks, biological systems, economics and social sciences. In particular, an important class of large-scale dynamical systems is that of dynamic games with a large number of minor agents in which each agent interacts with the average (or so-called mean-field) effect of other agents via couplings in their individual dynamics or individual cost functions. A minor agent is an agent which, asymptotically as the population size goes to infinity, has a negligible influence on the overall system while the overall population's effect on it is significant. For large population stochastic dynamic games with mean-field couplings and no major agent, the so-called mean-field game (MFG) theory was originally developed as a decentralized methodology in a series of papers by Huang together with Caines and Malhamé (see Huang et al. (2007, 2006), among others). For this class of game problems a closely related approach has been independently developed by Lasry and Lions (2007b). Carmona and Delarue (2013) develop a probabilistic approach to MFG problems using tailor-made forms of the stochastic maximum principle. Huang (2010b); Nguyen and Huang (2012); Nourian and Caines (2013) address mean-field game interactions in the presence of a major agent who has a strong influence on the minor agents. Since in these settings all minor agents respond to the same major agent, their mean-field is directly impacted by the major agent and hence is a random process even asymptotically as the population size of minor agents approaches 
infinity. This is in contrast to the situation in the standard MFG models with only minor agents. Carmona et al. (2016) provide an analysis of MFG problems with common noise and demonstrate existence and uniqueness. The reader is referred to the recent survey paper Caines (2014) and book Bensoussan et al. (2013) for some of the research on MFG theory and its applications.

The key idea of the MFG theory is to specify a certain equilibrium relationship between individual strategies and the overall effect of the population on a given agent, called the mass effect, as the population size $N$ goes to infinity. Specifically, in equilibrium: (i) the individual strategy of each agent is a best response to the infinite population mass effect in the sense of a so-called $\epsilon$-Nash equilibrium, and (ii) the set of strategies collectively replicates the same mass effect, this being a dynamic game theoretic fixed point property. The defining $\epsilon$-Nash equilibrium property of the MFG equilibrium indicates that any given agent can benefit at most by $\epsilon$ when deviating unilaterally from its mean-field game strategy. The estimates in Huang et al. $(2006,2007)$ show that $\epsilon=O(1 / \sqrt{N})$.

This paper introduces a MFG modeling framework for a multiple agent optimal execution problem with continuous trading. We generalize the classical single agent optimal liquidation problem (as in Almgren and Chriss (2001)) to a setting with (i) a major agent who is liquidating a large portion of shares, (ii) a number of minor agents (highfrequency traders (HFTs)) who detect and trade (optimally) along with the liquidator, and (iii) noise traders whose strategies are independent of the strategies that the major and minor agents use to trade. In this formulation all agents' (major, minor and noise) trades induce a permanent price impact on the fundamental asset price, and major/minor agents receive a temporary price impact when trading. Each agent aims to balance their price impact against price uncertainty and maximize profits all the while being subject to the action of other traders. This cross-interaction between the major and minor agents (and among the minor agents), through the impact that each trader has on the drift of the fundamental price, is a distinct feature of our setup.

Our formulation, therefore, falls within the realm of stochastic dynamic game problems with mean-field couplings in the dynamics. It is not feasible for each individual agent to track the actions of all other agents. To address this issue, we instead analyze the problem using MFG theory and obtain a set of decentralized feedback trading strategies for major and minor agents. The strategies can be explicitly obtained from a set of auxiliary stochastic optimal control problems which we explicitly solve. The MFG solution is then reduced to a deterministic fixed point problem - which we do not solve explicitly, but there are efficient numerical schemes for it. It is important to note that the asymptotic mean-field of HFTs is a "controlled mean-field" depending on the 


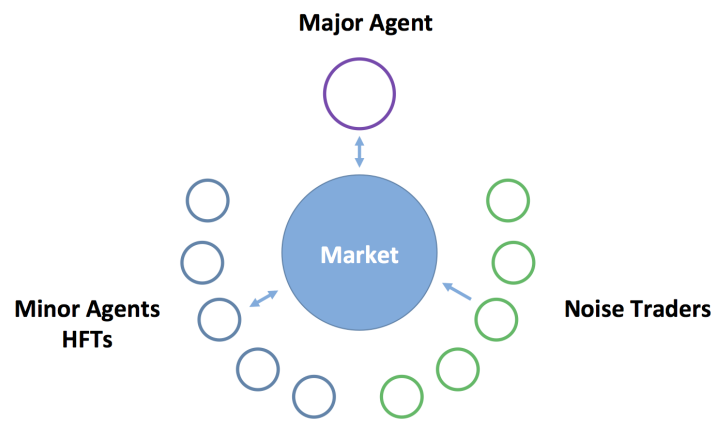

Figure 2.1: Interaction between major and minor agents through the market

major agent's trading strategy which is in contrast to both non-controlled deterministic mean-field of standard MFG problems in Huang et al. (2006, 2007). The resulting meanfield control laws yield a trading algorithm so that each individual agent may implement a decentralized strategy based on their local information together with information on the mean of minor agents' initial shares. We also show that for a finite $N$ population of HFTs, the set of major-minor agent MFG strategies has an $\epsilon_{N}$-Nash equilibrium property where $\epsilon_{N} \rightarrow 0$ as $N \rightarrow \infty$.

The organization of the paper is as follows. Section 2.2 is dedicated to the formulation of the major-minor agent optimal execution problem. A mean-field game (MFG) approach to the problem is presented in Section 2.3 which includes two auxiliary stochastic optimal control problems for major and minor agents in the mean-field limit and their solutions (Subsection 2.3.1 and 2.3.2), a MFG consistency condition (Subsetion 2.4.1). Section 2.5 shows the $\epsilon$-Nash equilibrium property of MFG decentralized trading strategies. Sample numerical simulations are given in Section 2.6, and some concluding remarks are provided in Section 2.7.

\subsection{Major-Minor Agent Optimal Execution Prob- lem}

We consider a dynamic game system involving: (i) a major institutional investor agent, (ii) a large population of minor agents as informed high frequency traders, and (iii) a large population of noise traders. A diagram of the interaction between major and minor agents through the market is shown in Fig. 2.1. Here, we show noise traders who buy and sell at "random" and add in additional order-flow. The description of each part of the model is given in detail below. 
Throughout we use the following notation. Let $\left(\Omega, \mathcal{F},\left\{\mathcal{F}_{t}\right\}_{t \geq 0}, \mathbb{P}\right)$ be a complete filtered probability space on which we have a standard $\mathbb{P}$-martingale $M=\left(M_{t}\right)_{t \in[0, T]}$ and a noise trader order-flow process $\mu=\left(\mu_{t}\right)_{t \in[0, T]}$ (assumed independent of $M$ ), and where $\mathcal{F}_{t}$ is the natural Filtration generated by $M$ and $\mu$. $\mathbb{E}$ denotes the expectation. The set of non-negative real numbers is denoted by $\mathbb{R}_{+} . T \in[0, \infty)$ denotes the terminal time. The integer $N$ is reserved to designate the population size of HFTs. The subscript $N$ for a process (such as state, control or wealth function) is used to indicate the dependence on the population size $N$. We use the superscript 0 for the major agent and an integer valued superscript for an individual minor agent. $\mathcal{C}_{b}(S)$ is the set of bounded and continuous functions on $S$. For two functions $f, g$ defined on some subset of $\mathbb{R}$ we use the notation $f(x)=O(g(x))$ if and only if there exists constants $C$ and $M$ such that $|f(x)| \leq C|g(x)|$ for all $x>M$.

\subsubsection{Trading Dynamics of Major and Minor Agents}

We let $q, q_{i}, y, u$ denote the inventory of the major agent, inventory of the $i^{\text {th }}$ minor agent, average inventory of the minor agents and the trading speed of noise traders respectively at time $t$ when time $t$ is explicitly given.

The major agent aims to liquidate her inventories $Q_{0}=\left(Q_{0, t}\right)_{t \in[0, T]}$ by the end of the trading horizon $T<+\infty$ by controlling her continuous rate of trading $\nu_{0}=\left(\nu_{0, t}\right)_{t \in[0, T]}$ so that

$$
d Q_{0, t}=\nu_{0, t} d t, \quad Q_{0,0} \gg 1, \quad 0 \leq t \leq T .
$$

Let $F=\left(F_{t}\right)_{t \in[0, T]}$ be the fundamental asset price (i.e. midprice of the asset) at time $t$ (in Section 2.2.3 we provide its model), then the execution price (i.e. the price at which the agent can sell the asset) the major agent receives from selling at time $t$ at the rate $\nu_{0, t}$ is

$$
S_{0, t}=F_{t}+a_{0} \nu_{0, t}, \quad 0 \leq t \leq T .
$$

Here, $a_{0}>0$ models the (linear) temporary price impact that the major agent's trading has on the price. The reader is referred to Jaimungal and Kinzebulatov (2014) and Cartea and Jaimungal (2016) for an illustration of how the parameter $a_{0}$ may be estimated from market data for the case of a single agent. The cash process dynamics of the major agent 
is given by

$$
d X_{0, t}=-S_{0, t} \nu_{0, t} d t, \quad 0 \leq t \leq T,
$$

with given $X_{0}(0)$ - typically set to 0 .

Next, consider a large number of minor agents who are opportunistic high frequency traders (HFTs) with the following dynamics for their inventories

$$
d Q_{i, t}=\nu_{i, t} d t, \quad 1 \leq i \leq N, \quad 0 \leq t \leq T,
$$

where $Q_{i}=\left(Q_{i, t}\right)_{t \in[0, T]}$ is the amount of shares of $i^{\text {th }}$ minor agent at time $t$ and $\nu_{i}=$ $\left(\nu_{i, t}\right)_{t \in[0, T]}$ is her trading rate-which can be positive or negative depending on whether the agent is selling or buying inventories, respectively. The set of initial shares of high frequency traders is given by $\left\{Q_{i}(0): 1 \leq i \leq N\right\}$. The execution price for the $i^{\text {th }}$ high frequency trader is given by

$$
S_{i, t}=F_{t}+a \nu_{i, t}, \quad 0 \leq i \leq N, \quad 0 \leq t \leq T
$$

where $a>0$ models the temporary impact that the trading of each minor agent has on the true price of the asset-note this may differ from, and is likely smaller than, the major agent's temporary impact parameter since the HFTs may be able to better take advantage of order book and order-flow information. The cash process dynamics of minor agents is given by

$$
d X_{i, t}=-S_{i, t} \nu_{i, t} d t, \quad 1 \leq i \leq N, \quad 0 \leq t \leq T,
$$

with given $X_{i, 0}$.

It should be noted that while a linear price impact model was assumed here, as in Almgren and Chriss (2001) and Obizhaeva and Wang (2013), more general limit order book dynamics have been looked at in Alfonsi and Schied (2010), Predoiu et al. (2011), and Cont and De Larrard (2013).

\subsubsection{Noise Traders}

We assume the noise trader dynamics are generated by a mean-reverting pure jump process where $\kappa$ is the mean-reverting rate, $\eta^{ \pm}$is the jump intensity, and $J_{t}^{ \pm}$are Poisson 
processes with parameter $\lambda^{ \pm}$

$$
d \mu_{t}=-\kappa \mu_{t} d t+\eta^{+} d J_{t}^{+}-\eta^{-} d J_{t}^{-}
$$

For notational simplicity, we assume that $\eta^{+}=\eta^{-}$and $\lambda^{+}=\lambda^{-}$. The analysis can be extended to the case where they are not equal in a straightforward manner.

Before proceeding, we required two assumptions on the initial inventories of the minor agents:

(A1) We assume that the initial inventories of minor agents $\left\{Q_{i, 0}: 1 \leq i \leq N\right\}$ are independent random variables. Moreover, there exists a constant $k$ independent of $N$ such that $\sup _{1 \leq i \leq N} \mathbb{E}\left|Q_{i, 0}\right|^{2} \leq k<\infty$.

(A2) There exists a distribution function $\mathbf{F}(q)$ on $\mathbb{R}$ such that the sample average of minor agents' initial shares converges almost surely to its expected value, i.e.

$$
\lim _{N \rightarrow \infty} \int_{\mathbb{R}} q d \mathbf{F}_{N}(q)=\int_{\mathbb{R}} q d \mathbf{F}(q),
$$

where $\mathbf{F}_{N}(q):=(1 / N) \sum_{i=1}^{N} 1_{\left\{Q_{i, 0}<q\right\}}$ denotes the set of empirical distribution functions.

If the sequence $\left\{Q_{i, 0}: 1 \leq i \leq N\right\}$ is generated by independent randomized observations on the distribution $\mathbf{F}$, then (A2) holds with probability one by the Strong Law of Large Numbers.

Remark: In this paper we assume homogenous minor agents with the same dynamical parameter $a$ in the execution price process (2.5). However, the modeling may be generalized to the case of heterogeneous minor agents with different temporary impact parameters $\left\{a_{i}: 1 \leq i \leq N\right\}$ as a sequence of independent random variables drawn from a known prior distribution.

\subsubsection{Fundamental Price Dynamics}

The fundamental asset price $F$ is assumed to satisfy the SDE

$$
d F_{t}=\left(\lambda_{0} \nu_{0, t}+\lambda \nu_{t}^{(N)}+\lambda_{\mu} \mu_{t}\right) d t+d M(t), \quad 0 \leq t \leq T,
$$

where $F(0)$ is given, $M=(M(t))_{0 \leq t \leq T}$ is a martingale (independent of initial inventories), $\nu^{(N)}:=\frac{1}{N} \sum_{j=1}^{N} \nu_{j}$ is the average trading of minor agents, $\mu=\left(\mu_{t}\right)_{0 \leq t \leq T}$ is the orderflow of noise traders, and $\lambda_{0}, \lambda \geq 0$ are the strength of the (linear) permanent impact 
of the major and minor agent's trading, respectively, on the fundamental price-if it is positive (more buyers than sellers), then prices are pushed upwards, if it is negative (more sellers than buyers), then prices are pushed downwards. The martingale term models the aggregate random impact of cancellations and posts in the limit order book on the fundamental price dynamics (see Fig. 2.1).

Here, the major and minor agents are coupled via the drift of the fundamental asset price in (2.8). While trading of each individual minor agent has an asymptotically negligible impact on the fundamental price, in the large $N$ population limit of HFTs, the aggregated of all minor agents have a non-negligible impact on the fundamental asset price process.

\subsubsection{The Agents' Performance Criteria}

The major agent's objective is to maximize her finite time horizon wealth, subject to an inventory penalty. Specifically, her performance criteria is given by

$$
J_{0}\left(\nu_{0} ; \nu_{-0}\right):=\mathbb{E}\left[X_{0, T}+Q_{0, T}\left(F_{T}-\alpha Q_{0, T}\right)-\phi \int_{0}^{T}\left(Q_{0, u}\right)^{2} d u\right]
$$

where $\nu_{-0}:=\left(\nu_{1}, \cdots, \nu_{N}\right)$ are the trading strategies of the minor agents, $X_{0, T}=X_{0,0}-$

$\int_{0}^{T} S_{0, u} \nu_{0, u} d u$ is major agent's total cash at maturity, and $\alpha$ and $\phi$ are positive scalars. The notation $J_{0}\left(\nu_{0} ; \nu_{-0}\right)$ indicates the dependence of the wealth function $J_{0}$ on $\nu_{0}$ and $\nu_{-0}$.

In (2.9), the term $Q_{0, T}\left(F_{T}-\alpha Q_{0, T}\right)$ corresponds to the liquidation value of assets remaining at the end of the trading horizon. The quadratic term acts to penalize non-zero terminal inventories, hence, as $\alpha \rightarrow \infty$, the optimal strategy will lead to zero inventories at the end of the trading horizon. The running penalty term $\phi \int_{0}^{T}\left(Q_{0, u}\right)^{2} d u$ acts as an urgency penalization. When $\phi$ is large, the agent attempts to liquidate inventories quickly, while when $\phi=0$, the agent is indifferent about holding inventories through time. Cartea et al. (2013) demonstrate that such penalties can be interpreted as stemming from the agent's aversion to model uncertainty (also known as ambiguity aversion).

In the sequel, we aim to maximize the performance criteria over a class of admissible trading strategies $\mathcal{A}$ defined as

$$
\mathcal{A}:=\left\{\nu \in \mathcal{C}_{b}(\mathbb{R}) \text { and s.t. } \nu(t) \text { is } \mathcal{G} \text {-adapted }\right\}
$$

where $\mathcal{G}=\left(\mathcal{G}_{t}\right)_{t \in[0, T]}$ and $\mathcal{G}_{t}=\mathcal{F}_{t} \vee \sigma\left(Q_{i, t}, i=0, \ldots, N\right)$, i.e., the filtration $\mathcal{G}$ is the filtration $\mathcal{F}$ expanded by the inventories of all agents. 
The minor agents' performance criteria is given by

$$
J_{i}\left(\nu_{i} ; \nu_{-i}\right):=\mathbb{E}\left[X_{i, t}+Q_{i, t}\left(F_{T}-\psi Q_{i, t}\right)\right], \quad 1 \leq i \leq N
$$

where $\nu_{-i}:=\left(\nu_{0}, \nu_{1}, \cdots, \nu_{i-1}, \nu_{i+1}, \cdots, \nu_{N}\right), X_{i, t}=X_{i, 0}-\int_{0}^{T} S_{i, u} \nu_{i, u} d u$ is the $i^{\text {th }}$ minor agent's total terminal cash, and $Q_{i, t}\left(F_{T}-\psi Q_{i, t}\right)$ is the value of closing her position at the end of the trading horizon, with the penalty parameter $\psi>0$. Analogous to the major agent, we take the minor agent's set of admissible strategies to be $\mathcal{A}$.

\subsection{A mean-field Game Approach}

The major-minor agent optimal execution problem (2.1)-(2.10) can be seen as a set of stochastic optimal control problems that are coupled via the fundamental asset price dynamics (2.8). However, in this formulation the control input of each individual agent depends on the trading rate of all other agents which are unknown a priori. The formulation (2.1)-(2.10) falls within a class of stochastic dynamic games with mean-field couplings in the dynamics of the fundamental asset price. This problem is intractable for a large $N$ population of minor agents since it is not feasible for each individual agent to collect detailed state information of all the other agents. To deal with this, we develop a mean-field game framework through the following steps

1) Infinite population limit of minor agents: In this step a Nash equilibrium is characterized by an equilibrium relationship between the individual strategies for major and minor agents and the overall trading effect of minor agents on a given agent through the fundamental asset price dynamics. This equilibrium relationship is described by a mean-field game consistency condition. To this end, we define the mean trading speed of the minor agents as

$$
\bar{\nu}=\lim _{N \rightarrow \infty} \frac{1}{N} \sum_{i=1}^{N} \nu_{i}
$$

and the mean inventory of the minor agents as

$$
\mathcal{Q}_{t}=\lim _{N \rightarrow \infty} \frac{1}{N} \sum_{i=1}^{N} Q_{i, t}
$$

2) $\epsilon$-Nash equilibrium for the finite population of minor agents: The set of decentralized mean-field game trading strategies (developed in Step i) establishes an 
$\epsilon_{N}$-Nash equilibrium for a finite $N$ population of minor agents where $\epsilon_{N}$ goes to zero asymptotically as $N$ approaches infinity.

In the remainder of this section we proceed to demonstrate the details of Step 1. First, we approximate the average trading of minor agents $\nu^{(N)}$ in the population limit (i.e. $N \rightarrow \infty$ ) by a process $\bar{\nu}$ with the postulated form

$$
\bar{\nu}_{t}=g_{0}(t)+g_{1}(t) Q_{0, t}+g_{2}(t) \mathcal{Q}_{t}+g_{3}(t) \mu_{t}
$$

where $g_{0}, \ldots, g_{3}$ are (continuous) deterministic functions of time to be characterized later via self consistency equations in section 2.4.1. Second, we formulate and solve two auxiliary stochastic optimal control problems for the major agent and a representative minor agent by replacing the finite population process $\nu^{(N)}$ in (2.8) with its mean-field $\bar{\nu}$. Finally, we present consistency requirements for the mean-field approximation such that the average trading rate of minor agents obtained from auxiliary stochastic optimal control problems coincides in the population limit with $\bar{\nu}$ assumed in (2.13). This is the so-called mean filed game consistency condition.

\subsubsection{Infinite Number of Minor Agents}

Problem I: Auxiliary Stochastic Optimal Control Problem of the Major Agent. By replacing the process $\nu^{(N)}$ in $(2.8)$ with $\bar{\nu}(\cdot)$ given in (2.13), we obtain the following auxiliary stochastic optimal control problem for the major (liquidating) agent:

$$
\begin{aligned}
d Q_{0, t} & =\nu_{0, t} d t \\
d \mathcal{Q}_{t} & =\bar{\nu}_{t} d t \\
& =\bar{\nu}_{t} d t \\
d \bar{F}_{t} & =\left(\lambda_{0} \nu_{0, t}+\lambda \bar{\nu}_{t}+\lambda_{\mu} \mu_{t}\right) d t+d M_{t} \\
& =\left(\lambda_{0} \nu_{0, t}+\lambda\left(g_{0}(t)+g_{1}(t) Q_{0, t}+g_{2}(t) \mathcal{Q}_{t}\right)+\left(\lambda_{\mu}+\lambda g_{3}(t)\right) \mu_{t}\right) d t+d M_{t} \\
\bar{F}_{0} & =F_{0} \\
d \bar{X}_{0, t} & =-\left(\bar{F}_{t}+a_{0} \nu_{0, t}\right) \nu_{0, t} d t \\
\bar{X}_{0,0} & =X_{0,0}, \\
\bar{J}_{0}\left(\nu_{0}\right) & :=\mathbb{E}\left[\bar{X}_{0, T}+Q_{0, T}\left(\bar{F}_{T}-\alpha Q_{0, T}\right)-\phi \int_{0}^{T}\left(Q_{0, u}\right)^{2} d u\right]
\end{aligned}
$$


where the fundamental asset price process has been modified to $\bar{F}=\left(F_{t}\right)_{t \in[0, T]}$ to reflect the response to the mean-field. The associated maximisation problem is given by

$$
\bar{J}_{0}=\sup _{\nu_{0} \in \mathcal{A}_{0}} \bar{J}_{0}\left(\nu_{0}\right)
$$

and if the supremum is attained in $\mathcal{A}$, then we denote the optimal strategy by $\nu_{0}^{*}$. The solution of this stochastic optimal control problem is provided in Section 2.3.2.

Problem II: Auxiliary Stochastic Optimal Control Problem of a Representative Minor Agent. We now solve the problem for a particular minor agent, given the strategy of the major agent and other minor agents. By taking the mean-field limit of the problem, we replace both the process $\nu^{(N)}$, the empirical average trading speed, in (2.8) with $\bar{\nu}$, the mean-field trading speed of all minor agents, given in (2.13) and $\nu_{0}$ by the optimal trading speed from Problem I, $\nu_{0}^{*}$. We then obtain the following auxiliary stochastic optimal control problem for the representative minor agent $i$ :

$$
\begin{aligned}
d Q_{i, t} & =\nu_{i, t} d t \\
d \bar{F}_{t} & =\left(\lambda_{0} \nu_{0, t}^{*}+\lambda \bar{\nu}_{t}+\lambda_{\mu} \mu_{t}\right) d t+d M_{t} \\
& =\left(\lambda_{0} \nu_{0, t}+\lambda\left(g_{0}(t)+g_{1}(t) Q_{0, t}+g_{2}(t) \mathcal{Q}_{t}\right)+\left(\lambda_{\mu}+\lambda g_{3}(t)\right) \mu_{t}\right) d t+d M_{t}, \quad \bar{F}_{0}=F_{0}, \\
d \bar{X}_{i, t} & =-\left(\bar{F}_{t}+a \nu_{i, t}\right) \nu_{i, t} d t \\
\bar{J}_{i}\left(\nu_{i}\right) & :=\mathbb{E}\left[\bar{X}_{i, t}+Q_{i, t}\left(\bar{F}_{T}-\psi Q_{i, t}\right)\right] .
\end{aligned}
$$

where the fundamental asset price process has been modified to $\bar{F}=\left(F_{t}\right)_{t \in[0, T]}$ to reflect the response to the minor agent's mean-field and the major agent's response to that mean-field. The associated maximisation problem is given by

$$
\bar{J}_{i}=\sup _{\nu_{i} \in \mathcal{A}_{i}} \bar{J}_{i}\left(\nu_{i}\right)
$$

and if the supremum is attained in $\mathcal{A}_{i}$, then we denote the optimal strategy by $\nu_{i}^{*}$. The solution of this stochastic optimal control problem is provided in Section 2.3.2. 


\subsubsection{Solutions to Limiting Stochastic Optimal Control Prob- lems for Major and Minor Agents}

This section presents solutions to the auxiliary stochastic optimal control problems (2.14) and (2.15) using dynamic programming principle.

Solution to Problem I. This problem falls within the class of standard optimal execution problems of a single agent with a time dependent drift in the fundamental price and time dependent permanent impact. For $x, f \in \mathbb{R}, q \in \mathbb{R}_{+}$and $0 \leq t \leq T$, we define the performance criteria under the arbitrary trading strategy $\nu_{0} \in \mathcal{A}$ as

$$
H_{0}^{\nu_{0}}(t, x, f, q, y, u)=\mathbb{E}_{t, x, f, q}\left[\bar{X}_{0, T}+Q_{0, T}\left(\bar{F}_{T}-\alpha Q_{0, T}\right)-\phi \int_{t}^{T}\left(Q_{0}(u)\right)^{2} d u\right]
$$

where $\mathbb{E}_{t, x, f, q, y, u}[\cdot]:=\mathbb{E}\left[\cdot \mid \bar{X}_{0, t}=x, \bar{F}_{t}=f, Q_{0, t}=q, \mathcal{Q}_{t}=y, \mu_{t}=u\right]$. The value function is then given by

$$
H_{0}(t, x, f, q, y, u):=\sup _{\nu_{0} \in \overline{\mathcal{A}}_{0}} H_{0}^{\nu_{0}}(t, x, f, q, y, u) .
$$

Theorem 2.3.1. Assume that the value function $H_{0}(t, x, f, q, y, u)$ for the stochastic optimal control problem (2.14) is a $C^{1}$ function of the variables $t, x, q, y$ and $u$, and $C^{2}$ in $f$. Then, the major agent's auxiliary value function (2.16) admits the ansatz

$$
H_{0}(t, x, f, q, y, u)=x+q f+h_{0}(t, y, u)+h_{1}(t, y, u) q+h_{2}(t) q^{2}
$$

where the functions $h_{0}, h_{1}$, and $h_{2}$ satisfy the coupled PDEs

$$
\begin{gathered}
\left(\partial_{t}+\lambda\left(g_{0}(t)+g_{2}(t) y+g_{3}(t) u\right) \partial_{y}+\mathcal{L}^{u}\right) h_{0}+\frac{1}{4 a_{0}} h_{1}^{2}=0 \\
\left(\partial_{t}+\lambda\left(g_{0}(t)+g_{2}(t) y+g_{3}(t) u\right) \partial_{y}+\mathcal{L}^{u}\right) h_{1}+\lambda g_{1}(t) \partial_{y} h_{0} \\
+\left(\lambda g_{0}(t)+\lambda g_{2}(t) y+\lambda g_{3}(t) u+\lambda_{\mu} u\right)+\frac{1}{2 a_{0}}\left(\lambda_{0}+2 h_{2}\right) h_{1}=0 \\
\partial_{t} h_{2}(t)+\lambda g_{1}(t) \partial_{y} h_{1}+\lambda g_{1}(t)+\frac{1}{4 a_{0}}\left(\lambda_{0}+2 h_{2}\right)^{2}-\phi=0
\end{gathered}
$$

with terminal conditions

$$
h_{0}(T, y, u)=h_{1}(T, y, u)=0, \quad \text { and } \quad h_{2}(T, y, u)=-\alpha .
$$

Moreover, the optimal trading rate in the major agent's auxiliary control problem is

$$
\nu_{0, t}^{*}=\frac{1}{2 a_{0}}\left[\left(\lambda_{0}+2 h_{2}(t)\right) q+h_{1}\left(t, \mathcal{Q}_{t}, \mu_{t}\right)\right]
$$


Proof. See 2.A.1

Solution to Problem II. This problem falls within the class of optimal execution problems for a single agent with a time dependent drift in the fundamental price of the asset, and zero permanent impact (in contrast to the auxiliary control problem for the major which has permanent impact). For $x, f, q \in \mathbb{R}$ and $0 \leq t \leq T$, we define the performance criteria for a generic minor agent $i=1, \ldots, N$ under the arbitrary trading strategy $\nu_{i} \in \mathcal{A}$ as

$$
H_{i}^{\nu_{i}}\left(t, x, f, q_{i}, q, y, u\right)=\mathbb{E}_{t, x, f, q_{i}, q, y, u}\left[\bar{X}_{i, t}+Q_{i, t}\left(\bar{F}_{T}-\psi Q_{i, t}\right)\right]
$$

where $\mathbb{E}_{t, x, f, q_{i}, q, y, u}[\cdot]:=\mathbb{E}\left[\cdot \mid \bar{X}_{i, t}=x, \bar{F}_{t}=f, Q_{i, t}=q_{i}, Q_{0, t}=q, \mathcal{Q}=y, \mu_{t}=u\right]$ and her value function is

$$
H_{i}\left(t, x, f, q_{i}, q, y, u\right):=\sup _{\nu_{i} \in \overline{\mathcal{A}}_{i}} H_{i}^{\nu_{i}}\left(t, x, f, q_{i}, q, y, u\right)
$$

Theorem 2.3.2. Assume that the value function $H_{i}\left(t, x, f, q_{i}, q, y, u\right)$ for the auxiliary stochastic optimal control problem (2.15) is a $C^{1}$ function of the variables $t, x, q, q_{i}, y$ and $u$, and $C^{2}$ function of variable $f$. Then, a generic minor agent's auxiliary value function (2.20) admits the ansatz

$$
H_{i}\left(t, x, f, q_{i}, q, y, u\right)=x+f q_{i}+l_{0}(t, q, y, u)+l_{1}(t, q, y, u) q_{i}-\frac{a}{\frac{a}{\psi}+T} q_{i}^{2}
$$

where $l_{0}$ and $l_{1}$ are deterministic functions of time $(t)$, the major agent's inventory $(q)$, the mean inventory of the minor agents ( $y$ ) and noise traders speed of trading ( $u)$. The optimal trading rate $\nu_{i}^{*}(i=1, \ldots, N)$ in feedback form is given by

$$
\nu_{i}^{*}\left(t, q_{i}, q, y, u\right)=-\frac{q_{i, t}}{\frac{a}{\psi}+(T-t)}+\frac{1}{2 a} l_{1}(t, y, u) .
$$

Proof. See 2.A.2 


\subsubsection{Verification}

The control derived from the HJB equations are stochastic in general, due to the adapted nature of the solutions $\nu_{0}^{*}, \nu_{i}^{*}$. We make a further ansatz

$$
\begin{aligned}
& h_{0}(t, y, u)=h_{0}^{0}(t)+h_{0}^{1}(t) y+h_{0}^{2}(t) y^{2}+h_{0}^{\mu}(t) u y+h_{0}^{0, \mu} u+h_{0}^{2, \mu} u^{2} \\
& h_{1}(t, y, u)=h_{1}^{0}(t)+h_{1}^{1}(t) y+h_{1}^{\mu}(t) u
\end{aligned}
$$

and

$$
\begin{aligned}
& l_{0}(t, q, y, u)=l_{0}^{0}(t)+l_{0}^{1}(t) y+l_{0}^{2}(t) y^{2} \cdots+l_{0}^{0, \mu} u+l_{0}^{2, \mu} u^{2} \\
& l_{1}(t, q, y, u)=l_{1}^{0}(t)+l_{1}^{1}(t) q+l_{1}^{2}(t) y+l_{1}^{\mu}(t)
\end{aligned}
$$

where $l_{0}(t, q, y, u)$ is a quadratic function of $q, y, u$. This leads to the ODEs $(2.46),(2.51)$ in Appendix 2.A.2 which are at most quadratic in the state variables. Therefore, we have that if the system of coupled ODEs admits a solution, then

- for each $t$, the functions $H_{0}(t, x, f, q, y, u), H_{i}\left(t, x, f, q_{i}, q, y, u\right)$ are a $C^{2}$ map into $\mathbb{R}$

- the partial derivatives of $H_{0}(t, x, f, q, y, u), H_{i}\left(t, x, f, q_{i}, q, y, u\right)$ are continuous $\mathcal{F}_{t}$ adapted processes

In the absence of noise traders (jumps), the dynamics are a continuous stochastic process, and so the assumptions of Peng (1992) are satisfied implying that

$$
\begin{gathered}
J_{0}\left(\nu_{0}^{*}, \nu_{-0}^{*}\right)=\sup _{\nu^{0}} J_{0}\left(\nu_{0}, \nu_{-0}^{*}\right) \\
J_{i}\left(\nu_{i}^{*}, \nu_{-i}^{*}\right)=\sup _{\nu^{i}} J_{i}\left(\nu_{i}, \nu_{-i}^{*}\right)
\end{gathered}
$$

Therefore, the optimal controls derived through the HJB equation do indeed maximize the performance criteria for each agent.

With the inclusion of noise traders, and with the minor agents strategies being $\mathcal{F}_{t^{-}}$ predictable, where $\bar{\nu}$ is linear in the state variables, the problem is reduced to that of a jump-diffusion stochastic control problem. The verification theorem then still holds by an application of Theorem 9.1 in Øksendal and Sulem (2009). 


\section{$2.4 \quad$ Fixed Point Equations}

\subsubsection{Mean-Field Game Fixed Point}

When the mean-field game strategies for the minor agents are employed, they must be consistent with the ansatz assumed in (2.13) at the beginning. The mean-field strategy based on the form of (2.21) combined with (2.51) is

$$
\bar{\nu}(t, q, y, u)=\lim _{n \rightarrow \infty} \frac{1}{N} \sum_{i=1}^{N} \nu_{i}(t, q, y, u)=-\frac{y}{\frac{a}{\psi}+(T-t)}+\frac{1}{2 a}\left(l_{1}^{0}(t)+l_{1}^{1} q+l_{1}^{2} y+l_{1}^{\mu}(t) u\right)
$$

which, when compared to (2.13), yields the following set of consistency equations:

$$
\begin{aligned}
& g_{0}(t)=\frac{1}{2 a} l_{1}^{0}(t) \\
& g_{1}(t)=\frac{1}{2 a} l_{1}^{1}(t) \\
& g_{2}(t)=-\left(\frac{a}{\psi}+(T-t)\right)^{-1}+\frac{1}{2 a} l_{1}^{2}(t) \\
& g_{3}(t)=\frac{1}{2 a} l_{1}^{\mu}(t)
\end{aligned}
$$


By substituting (2.23) back into the following coupled ODEs

$$
\begin{aligned}
& \partial_{t} h_{0}^{0}+g_{0} h_{0}^{1}+\frac{1}{4 a_{0}}\left(h_{1}^{0}\right)^{2}=0, \quad h_{0}^{0}(T)=0, \\
& \partial_{t} h_{0}^{1}+2 g_{0} h_{0}^{2}+g_{2} h_{0}^{1}+\frac{1}{2 a_{0}} h_{1}^{0} h_{1}^{1}=0, \quad h_{0}^{1}(T)=0, \\
& \partial_{t} h_{0}^{2}+2 g_{2} h_{0}^{2}+\frac{1}{4 a_{0}}\left(h_{1}^{1}\right)^{2}=0, \quad h_{0}^{2}(T)=0, \\
& \left(\partial_{t}-\kappa\right) h_{0}^{\mu}+2 g_{3} h_{0}^{2}+\frac{1}{2 a_{0}} h_{1}^{\mu} h_{1}^{1}=0, \quad h_{0}^{\mu}(T)=0, \\
& \left(\partial_{t}+\lambda g_{3} h_{0}^{\mu}-\kappa\right) h_{0}^{0, \mu}+\frac{1}{2 a_{0}} h_{1}^{0} h_{1}^{\mu}=0, \quad h_{0}^{0, \mu}(T)=0, \\
& \left(\partial_{t}-\kappa\right) h_{0}^{2, \mu}+\frac{1}{2 a_{0}} h_{1}^{1} h_{1}^{\mu}=0, \quad h_{0}^{2, \mu}(T)=0, \\
& \partial_{t} h_{1}^{0}+g_{0} h_{1}^{1}+h_{0}^{1} g_{1}+\lambda g_{0}+\frac{1}{2 a_{0}}\left(\lambda_{0}+2 h_{2}\right) h_{1}^{0}=0, \quad h_{1}^{0}(T)=0, \\
& \partial_{t} h_{1}^{1}+2 h_{0}^{2} g_{1}+\lambda g_{2}+\frac{1}{2 a_{0}}\left(\lambda_{0}+2 h_{2}+2 a_{0} g_{2}\right) h_{1}^{1}=0, \quad h_{1}^{1}(T)=0, \\
& \left(\partial_{t}-\kappa\right) h_{1}^{\mu}+g_{3} h_{1}^{1}+\left(\lambda g_{3}+\lambda_{\mu}\right)+\frac{1}{2 a_{0}}\left(\lambda_{0}+2 h_{2}\right) h_{1}^{\mu}=0, \quad h_{1}^{\mu}(T)=0 \\
& \partial_{t} l_{1}^{0}(t)+g_{0}(t) l_{1}^{2}(t)+\frac{h_{1}^{0}(t)}{2 a_{0}}\left(\lambda_{0}+l_{1}^{0}(t)\right)+\frac{1}{a}\left(l_{1}^{0}(t) l_{2}(t)\right)+\lambda g_{0}(t)=0, \quad l_{1}^{0}(T)=0 \\
& \partial_{t} l_{1}^{1}(t)+\frac{1}{a}\left(l_{1}^{1}(t) l_{2}(t)\right)+g_{1}(t)\left(\lambda+l_{1}^{2}(t)\right)+\frac{h_{1}^{1}(t)}{2 a_{0}}\left(l_{1}^{1}(t)+\lambda_{0}\right)=0, \quad l_{1}^{1}(T)=0 \\
& \partial_{t} l_{1}^{2}(t)+\frac{1}{a}\left(l_{1}^{2}(t) l_{2}(t)\right)+g_{2}(t) l_{1}^{2}(t)+\frac{\lambda_{0}+2 h_{2}}{2 a_{0}}\left(\lambda_{0}+l_{1}^{2}(t)\right)+\left(\lambda g_{2}(t)\right)=0, \quad l_{1}^{2}(T)=0 \\
& \left(\partial_{t}-\kappa\right)\left(l_{1}^{\mu}(t)+g_{3}(t)\right) l_{1}^{2}(t)+\frac{h_{1}^{\mu}(t)}{2 a_{0}} l_{1}^{\mu}(t)+\frac{1}{a} l_{1}^{\mu}(t) l_{2}(t)+\lambda g_{3}(t)+\lambda_{\mu}=0, \quad l_{1}^{\mu}(T)=0
\end{aligned}
$$

we see that the fixed point strategy is a solution to a series of coupled Riccatti ODEs.

\subsubsection{Behaviour of the mean-field Game System in the Infinite Penalty Set-up}

In this section we consider the trading strategy for the major and minor agents when the minor agents have infinite temporary impact, (i.e., as $a \rightarrow \infty$ ). In this limit, we anticipate that the minor agents will not trade, since trading has an infinite cost.

Lemma 2.4.1. In the limit, as $a \rightarrow \infty$, we have that $\nu_{i}^{*} \rightarrow 0$.

Proof. Given a solution to (2.51) with $a \rightarrow \infty$ when substituting (2.23) and $g_{i}(t)=0$ for $i=1,2,3,4$ into the ODEs, we can see that for any bounded solutions $l_{1}^{0}, l_{1}^{1}, l_{1}^{2}, l_{1}^{\mu}$, we 
have that (2.23) gives us $g_{i}(t)=0$ for $i=1,2,3,4$ as $a \rightarrow \infty$, which gives shows that the consistency equations are satisfied.

As a result, there is no mean field and so the major agent's optimal strategy is the solution to the PDEs

$$
\begin{aligned}
\left(\partial_{t}+\mathcal{L}^{\mu}\right) h_{0}(t, y, u)+\frac{1}{4 a_{0}} h_{1}(t, y, u)^{2} & =0, & h_{0}(T, y, u)=0 \\
\left(\partial_{t}+\mathcal{L}^{\mu}\right) h_{1}(t, y, u)+\lambda_{\mu} u+\frac{1}{2 a_{0}}\left(\lambda_{0}+2 h_{2}(t)\right) h_{1}(t, y, u) & =0, & h_{1}(T, y, u)=0 \\
\partial_{t} h_{2}(t)+\frac{1}{4 a_{0}}\left(\lambda_{0}+2 h_{2}(t)\right)^{2}-\phi & =0, & h_{2}(T)=-\alpha .
\end{aligned}
$$

which is the same as the equations found in Cartea and Jaimungal (2016) - where the authors study the effect of order-flow in an optimal execution problem for a single agent.

\subsection{Decentralized mean-field Game Trading Strate- gies: The $\epsilon_{N}$-Nash Equilibrium Property}

In this section, we will prove that the decentralized mean-field solution has the $\epsilon_{N}$-Nash equilibrium property in the absence of noise traders. To this end, we make the following assumption.

(A3) Assume there exists a unique solution set $\left(g_{0}, g_{1}, g_{2}\right)$ in $\mathcal{C}_{b}\left(\mathbb{R}_{+}\right)$to the mean-field game system (2.23).

Under (A3), the mean-field game trading strategies for a finite population of major and minor agents are given by

$$
\begin{array}{ll}
\nu_{0, t}^{*}=\frac{1}{2 a_{0}}\left(\lambda_{0}+2 h_{2}(t)\right) Q_{0, t}^{*}+\frac{1}{2 a_{0}} h_{1}\left(t, Q_{0, t}^{*}, \frac{1}{N} \sum_{i=1}^{N} Q_{i, t}^{*}\right), & \text { and } \\
\nu_{i, t}^{*}=-\frac{\psi}{a+\psi(T-t)} Q_{i, t}^{*}+\frac{1}{2 a} l_{1}\left(t, Q_{0, t}^{*}, \frac{1}{N} \sum_{i=1}^{N} Q_{i, t}^{*}\right), & i \in\{1, \ldots, N\},
\end{array}
$$

where $h_{1}(\cdot)$ and $h_{2}(\cdot)$ are determined by $(2.25 \mathrm{~b})$ and $(2.25 \mathrm{c})$, respectively, and $l_{1}(\cdot)$ is given in (2.50b). Note that in equations (2.25b) and (2.25c), the functions $g_{0}, g_{1}, g_{2}$, and $g_{3}$ are the unique solution of the mean-field game system (2.23). The implementation of the overall mean-field game trading rates for the major and minor agents (2.26) have a decentralized form. That is, each agent enacts a control law which consists of the feedback of its own quantity of shares plus a pre-computed deterministic effect due to the cross interaction of major-minor agents. 
Applying the optimal trading strategies (2.26) in a finite population of minor agents (2.1)-(2.6) yields the following

$$
\begin{aligned}
d Q_{0, t}^{*} & =\nu_{0, t}^{*} d t, & & Q_{0,0}^{*}=Q_{0,0} \\
d X_{0, t}^{*} & =-\left(F_{t}^{*}+a_{0} \nu_{0, t}^{*}\right) \nu_{0, t}^{*} d t, & & X_{0,0}^{*}=X_{0,0}
\end{aligned}
$$

and for $i \in 1, \ldots, N$,

$$
\begin{aligned}
d Q_{i, t}^{*} & =\nu_{i, t}^{*} d t, & Q_{i, 0}^{*} & =Q_{i, 0} \\
d X_{i, t}^{*} & =-\left(F_{t}^{*}+a \nu_{i, t}^{*}\right) \nu_{i, t}^{*} d t & X_{i, 0}^{*} & =X_{i, 0}
\end{aligned}
$$

where the agents optimal behavior are coupled through the optimal trading strategies in (2.26) and the fundamental asset price dynamics

$$
d F_{t}^{*}=\left(\lambda_{0} \nu_{0, t}^{*}+\frac{\lambda}{N} \sum_{i=1}^{N} \nu_{i, t}^{*}\right) d t+d M_{t}, \quad F_{t}^{*}=F_{0} .
$$

Definition 2.5.1. Given $\epsilon>0$, the set of trading rates $\left\{\nu_{i}^{*} \in \mathcal{A}_{i}: 0 \leq i \leq N\right\}$ for $N+1$ major and minor agents generates an $\epsilon$-Nash equilibrium with respect to the utility functions $\left\{J_{i}: 0 \leq i \leq N\right\}$, if

$$
J_{i}\left(\nu_{i}^{*}, \nu_{-i}^{*}\right) \leq \sup _{\nu_{i} \in \mathcal{A}_{i}} J_{i}\left(\nu_{i}, \nu_{-i}^{*}\right) \leq J_{i}\left(\nu_{i}^{*}, \nu_{-i}^{*}\right)+\epsilon,
$$

for any $1 \leq i \leq N$.

The $\epsilon$-Nash equilibrium property of the trading rates $\left\{\nu_{i}^{o} \in \mathcal{A}_{i}: 0 \leq i \leq N\right\}$ implies that for any given $\epsilon>0$, there exists $M_{\epsilon}$ such that for any population size of minor agents $N \geq M_{\epsilon}$, any agent $i$ within the population can only benefit $\epsilon$ by unilaterally (i.e. while any other agent $j, 0 \leq j \neq i \leq N$, employes $\nu_{j}^{o}$ ) deviating from its trading strategy $\nu_{i}^{o}$.

We denote

$$
\epsilon_{N}:=\left|\frac{1}{N} \sum_{i=1}^{N} Q_{i, 0}-\mathcal{Q}_{0}\right| \equiv\left|\int_{\mathbb{R}} q d \mathbf{F}_{N}(q)-\int_{\mathbb{R}} q d \mathbf{F}(q)\right|
$$

where $\mathcal{Q}_{0}$ is the average of the distribution employed in the MFG, where the distributions $\mathbf{F}_{N}$ and $\mathbf{F}$ are defined in (A2). It is evident that under (A2) $\lim _{N \rightarrow \infty} \epsilon_{N}=0$.

To facilitate the estimate of the bounds on the approximate finite game solution, we construct several auxiliary processes. First, introduce the auxiliary cash dynamics $\bar{X}_{-i}^{*}=\left(\bar{X}_{-i, t}^{*}\right)_{t \in[0, T]}$ for the $N$ minor agents, where the fundamental price follows the 
response to the optimal mean-field strategy, but the cash accumulates according to an arbitrary strategy from the $i$-th agent. Specifically, $\bar{X}_{-i}$ satisfy the SDE

$$
d \bar{X}_{-i, t}^{*}=-\left(\bar{F}_{t}^{*}+a \nu_{i, t}\right) \nu_{i, t} d t, \quad \bar{X}_{-i, 0}^{*}=X_{i, 0}, \quad 1 \leq i \leq N,
$$

for an arbitrary $\nu_{i} \in \mathcal{A}_{i}$, and where $\bar{F}^{*}=\left(\bar{F}_{t}^{*}\right)_{t \in[0, T]}$ is the response of the fundamental price to the mean-field strategy $\bar{\nu}_{t}^{*}:=g_{0}(t)+g_{1}(t) Q_{0, t}^{*}+g_{2}(t) \mathcal{Q}_{t}^{*}$, where $Q_{0, t}^{*}$ and $\mathcal{Q}_{t}^{*}$ are the major player's inventory, and the average of the minor players' inventories in the mean-field game.

$$
d \bar{F}_{t}^{*}=\left\{\left(\lambda_{0} \nu_{0, t}^{*}+\lambda\left(g_{0}(t)+g_{1}(t) Q_{0, t}^{*}+g_{2}(t) \mathcal{Q}_{t}^{*}\right)\right\} d t+d M_{t}, \quad \bar{F}^{*}(0)=F_{0} .\right.
$$

It is important to note that in (2.31) the infinite population mean-field process appears and not the finite population average of the minor agents' mean-field controls $\frac{1}{N} \sum_{i=1}^{N} \nu_{i}^{*}(t)$ in $(2.28)$.

Next, we introduce the cash processes subject to the optimal mean-field strategies $\bar{X}_{i}^{*}=\left(\bar{X}_{i, t}^{*}\right)_{t \in[0, T]}$ which satisfy the SDEs

$$
\begin{aligned}
& d \bar{X}_{0}^{*}(t, q, y)=-\left(\bar{F}_{t}^{*}+a_{0} \nu_{0, t}^{*}\right) \nu_{0, t}^{*} d t, \quad \bar{X}_{0}^{*}(0)=X_{0,0} \\
& d \bar{X}_{i}^{*}\left(t, q_{i}, q, y\right)=-\left(\bar{F}^{*}(t)+a \nu_{i}^{*}\left(t, q_{i}, q, y\right)\right) \nu_{i}^{*}\left(t, q_{i}, q, y\right) d t \quad \bar{X}_{i}^{*}(0)=X_{i, 0}, \quad 1 \leq i \leq N .
\end{aligned}
$$

Note the difference between $\bar{X}_{-i}^{*}$ and $\bar{X}_{i}^{*}$ is that in $\bar{X}_{-i}^{*}$ the arbitrary strategy $\nu_{i} \in \mathcal{A}_{i}$ is used to generate the cash process, while in $\bar{X}_{i}^{*}$, the optimal mean-field strategy $\nu_{i}^{*}$ is used to generate the cash process, while in both cases, the fundamental price is the one generated by the mean-field strategy.

Continuing with defining additional auxiliary process, for a given $\nu_{0} \in \mathcal{A}_{0}$, we let $\bar{F}_{-0}^{*}=\left(\bar{F}_{-0}^{*}(t)\right)_{t \in[0, T]}$ denote the response of the fundamental price to the mean-field of the minor agents, but the major agent acting with the arbitrary strategy $\nu_{0}$. We further let $\bar{X}_{-0}^{*}=\left(\bar{X}_{-0}^{*}(t)\right)_{t \in[0, T]}$ denote the major agent's cash process in response to this fundamental price, and with the same arbitrary strategy $\nu_{0}$. In all, they satisfy the 


\section{SDEs}

$$
\begin{aligned}
d \bar{F}_{-0}^{*}(t, q, y) & =\left(\lambda_{0} \nu_{0}(t, q, y)+\lambda \bar{\nu}^{*}(t, q, y)\right) d t+d M_{t} & \\
& =\left(\lambda_{0} \nu_{0}(t, q, y)+\lambda\left(g_{0}(t)+g_{1}(t) q+g_{2}(t) y\right)\right) d t+d M_{t} & \\
d \bar{X}_{-0}^{*}(t, q, y) & =-\left(\bar{F}_{-0}^{*}(t, q, y)+a_{0} \nu_{0}(t, q, y)\right) \nu_{0}(t, q, y) d t, & \bar{F}_{-0}^{*}(t)=F_{0}
\end{aligned}
$$

where $\bar{\nu}^{*}(\cdot) \equiv g_{0}(\cdot)+g_{1}(\cdot) Q_{0}(\cdot)+g_{2}(\cdot) \mathcal{Q}(\cdot)$ is the infinite population mean-field of minor agents.

Finally, we introduce the auxiliary fundamental price dynamics and cash processes in response to the finite population actions. To distinguish them in notation from the above, there are no overlines on the variables. So that $F_{-i}^{*}=\left(F_{-i}^{*}(t)\right)_{t \in[0, T]}$, for $i=0, \ldots, N$, denotes the fundamental price in response to finite population action of all agents, excluding the $i^{\text {th }}$ agent who acts according to an arbitrary strategy, acting according to the mean-field strategies. Specifically, we have

$$
\begin{array}{rr}
d F_{-0}^{*}(t, q, y)=\left(\lambda_{0} \tilde{\nu}_{0}(t, q, y)+\frac{\lambda}{N} \sum_{i=1}^{N} \nu_{i}^{*}(t, q, y)\right) d t+d M_{t}, & F_{-0}^{*}(t)=F_{0} \\
d F_{-i}^{*}(t, q, y)=\left(\lambda_{0} \tilde{\nu}_{0}^{*}(t, q, y)+\frac{\lambda}{N} \sum_{j=1, j \neq i}^{N} \nu_{j}^{*}(t, q, y)+\frac{\lambda}{N} \nu_{i}(t, q, y)\right) d t+d M_{t}, \quad F_{-i}^{*}(t)=F_{0} .
\end{array}
$$

for given $\nu_{0} \in \mathcal{A}_{0}$ and $\nu_{i} \in \mathcal{A}_{i}, 1 \leq i \leq N$. Similarly, we let $X_{-i}^{*}=\left(X_{-i}^{*}((t))_{t \in[0, T]}\right.$, for $i=0, \ldots, N$, denote agent's $i$ 's cash process in response to finite population action of all agents, excluding the $i^{\text {th }}$ agent who acts according to an arbitrary strategy, acting according to the mean-field strategies. Specifically, they satisfy the SDEs

$$
\begin{array}{cc}
d X_{-0}^{*}(t, q, y)=-\left(F_{-0}^{*}(t, q, y)+a_{0} \nu_{0}(t, q, y)\right) \nu_{0}(t, q, y) d t, & X_{-0}^{*}(0)=X_{0,0} \\
d X_{-i}^{*}\left(t, q_{i}, q, y\right)=-\left(F_{-i}^{*}(t, q, y)+a \nu_{i}\left(t, q_{i}, q, y\right)\right) \nu_{i}\left(t, q_{i}, q, y\right) d t, \quad X_{-i}^{*}(0)=X_{i, 0}, & 1 \leq i \leq N .
\end{array}
$$

Given all of these auxiliary processes, we have the following bounds on the deviations of the various fundamental prices and cash processes. 
Lemma 2.5.1. Assume (A1)-(A3) hold. Then, for any $0 \leq t \leq T$ we have

$$
\begin{aligned}
& \mathbb{E}\left|F_{-i}^{*}(t)-\bar{F}^{*}(t)\right|=O\left(\epsilon_{N}\right)+O\left(\frac{1}{N}\right), \quad \mathbb{E}\left|X_{-i}^{*}(t)-\bar{X}_{-i}^{*}(t)\right|=O\left(\epsilon_{N}\right)+O\left(\frac{1}{N}\right), \quad 1 \leq i \leq N \\
& \mathbb{E}\left|F_{-0}^{*}(t)-\bar{F}_{-0}^{*}(t)\right|=O\left(\epsilon_{N}\right), \quad \mathbb{E}\left|X_{-0}^{*}(t)-\bar{X}_{-0}^{*}(t)\right|=O\left(\epsilon_{N}\right),
\end{aligned}
$$

where $\epsilon_{N}$ is defined in (2.29), and $\bar{X}_{-i}^{*}(\cdot), \bar{F}^{*}(\cdot), \bar{F}_{-0}^{*}(\cdot), \bar{X}_{-0}^{*}(\cdot), F_{-0}^{*}(\cdot), F_{-i}^{*}(\cdot), X_{-0}^{*}(\cdot)$ and $X_{-i}^{*}(\cdot)$ are given in (2.30)-(2.39), respectively.

Proof. Proof in 2.B.1

Armed with these bounds, we are now in a position to state the main result of this section.

Theorem 2.5.1. Assume (A1)-(A3) hold. Then, the set of mean-field game trading strategies $\left\{\nu_{i}^{*} \in \mathcal{A}_{i}: 0 \leq i \leq N\right\}$ in (2.26a)-(2.26b) generates an $\epsilon$-Nash equilibrium, i.e. for any fixed $0 \leq i \leq N$ we have

$$
J_{i}\left(\nu_{i}^{*}, \nu_{-i}^{*}\right) \leq \sup _{\nu_{i} \in \mathcal{A}_{i}} J_{i}\left(\nu_{i}, \nu_{-i}^{*}\right) \leq J_{i}\left(\nu_{i}^{*}, \nu_{-i}^{*}\right)+O\left(\epsilon_{N}\right)+O\left(\frac{1}{N}\right)
$$

where $\epsilon_{N}$ is given in (2.29), and $\lim _{N \rightarrow \infty} \epsilon_{N}=0$ (a.s.).

Proof. Proof in 2.B.2.

We note that the $\epsilon$-Nash equilibrium result proved here differs from some of the results in the literature, as this $\epsilon$-Nash equilibrium holds with respect to the N-player empirical average, rather than the infinite player mean-field average. A similar result should hold if the infinite-player mean-field is used as well.

\subsection{Simulations}

(The parameters which were calibrated for the simulation can be found in the appendix)

\subsubsection{Major and minor agents with same liquidation horizon}

In the following figures, the broker's inventory is plotted with respect to execution time. In the top figure, the absolute inventory level is plotted, while the second figure describes the difference in inventory when the broker uses the MFG strategy and when they ignore 
the presence of HFT traders. In comparison to Cartea and Jaimungal (2016), the trading speed of the major agent initially speeds up at the start and end of the liquidation period in order to take advantage of the relatively higher stock price, and slows down in the middle to minimize the amount of stocks sold when the mid-price is at its lowest point. Additionally, we can see that while the mean-field execution strategy starts off with a lower average execution price, it makes up for this setback later on when the minor agent needs to buy back stock.
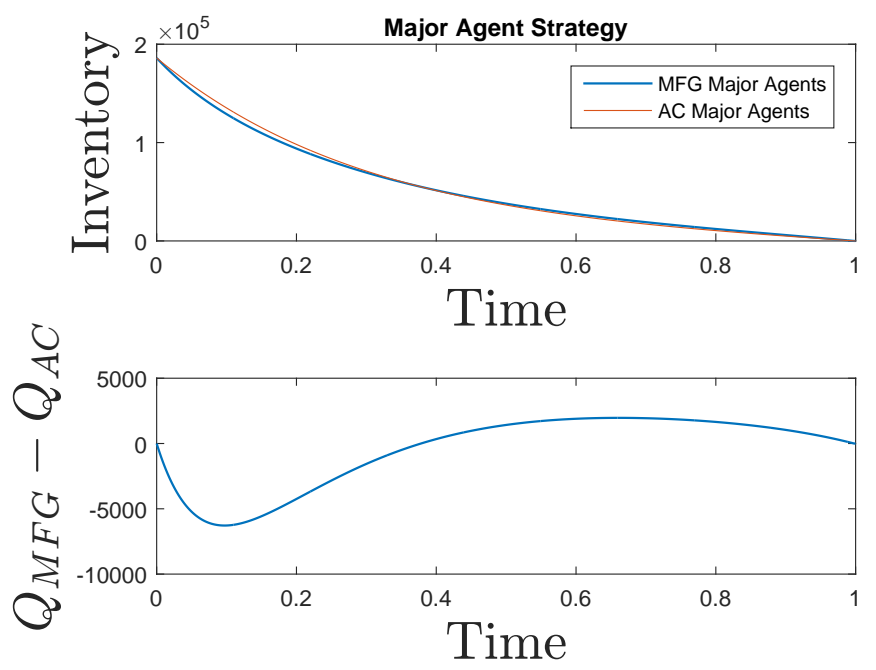

Figure 2.2: Optimal Trading for major agent in the presence of Order Flow and HighFrequency Traders

A similar simulation was performed for the minor HFT agents. Their trading strategy consists of shorting stock in order to push the price down, and then slowly purchasing the stock back, taking advantage of the continued downward pressure on price from the broker liquidating their portfolio.

The PnL distribution is positive with only a few stocks having a chance to underperform the Almgren-Criss strategy. A linear regression of the PnL distribution on the variable $\frac{\lambda_{0}}{a_{0}}$ gives

$$
\operatorname{PnL} \approx 0.584 \frac{\lambda_{0}}{a_{0}}
$$

In the Table 2.2, the increase in profit is plotted based on the advantage in basis points. The equation used is

$$
\mathrm{PnL}=0.01 \times \frac{X_{M F G}-X_{A C}}{X_{A C}}
$$



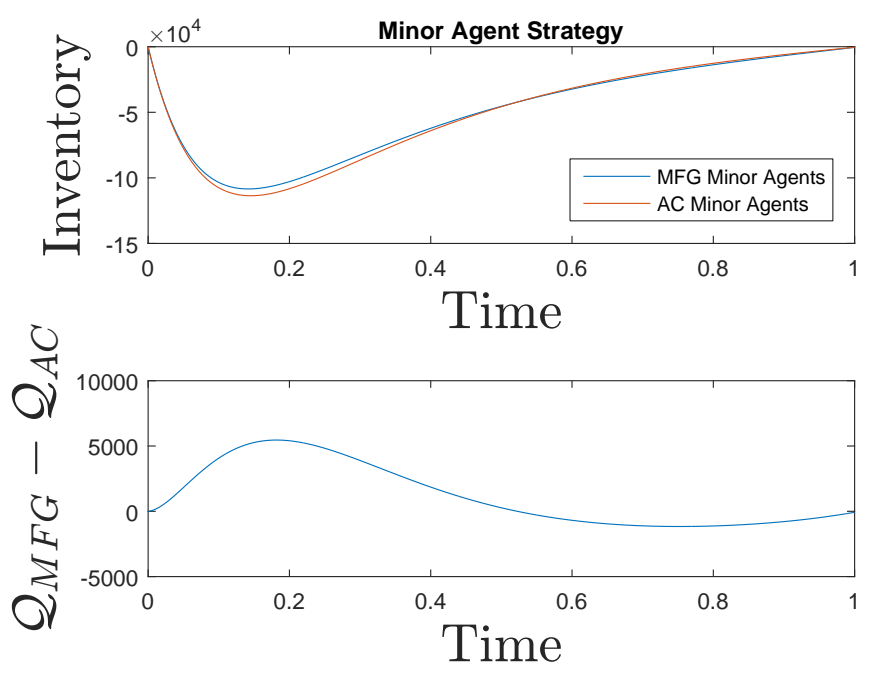

Figure 2.3: Optimal Trading for minor agents

Table 2.1: Least Squares Regression Output

\begin{tabular}{rrrrr}
\hline & Coefficients & Standard Error & $t$ Stat & P-value \\
\hline Intercept & -0.054 & 0.303 & -0.177 & 0.862 \\
Slope & 0.584 & 0.151 & 3.861 & 0.002 \\
\hline
\end{tabular}

where $X_{M F G}$ is the wealth of the broker under the mean-field game strategy, and $X_{A C}$ is the wealth of the broker under the strategy from Almgren and Chriss (2001). The term $\frac{\lambda_{0}}{a_{0}}$ is the relative size of the permanent impact to the temporary impact, so a higher value would allow minor agents to take better advantage of the major agent's liquidation strategy. 
Table 2.2: Mean-Field vs Order-Flow strategy with liquidation penalty for Minor Agents (20\% of Order Flow)

\begin{tabular}{|c|c|c|c|c|c|c|c|c|}
\hline \multirow[b]{2}{*}{ Ticker } & \multirow[b]{2}{*}{ Mean } & \multirow[b]{2}{*}{ StDev } & \multicolumn{5}{|c|}{ Quantiles } & \multirow[b]{2}{*}{$\mathbb{P}(M F G<A C$} \\
\hline & & & $5 \%$ & $25 \%$ & $50 \%$ & $75 \%$ & $95 \%$ & \\
\hline EBAY & 1.37 & 0.60 & 0.39 & 0.96 & 1.37 & 1.78 & 2.37 & $1 \%$ \\
\hline INTC & 1.69 & 0.71 & 0.53 & 1.21 & 1.70 & 2.17 & 2.87 & $1 \%$ \\
\hline DOW & 1.54 & 0.92 & 0.02 & 0.91 & 1.53 & 2.15 & 3.06 & $5 \%$ \\
\hline $\mathrm{CSCO}$ & 1.22 & 0.62 & 0.21 & 0.80 & 1.22 & 1.64 & 2.25 & $2 \%$ \\
\hline COST & 0.88 & 0.51 & 0.06 & 0.53 & 0.87 & 1.21 & 1.72 & $4 \%$ \\
\hline AXP & 0.73 & 0.64 & -0.32 & 0.31 & 0.73 & 1.15 & 1.77 & $13 \%$ \\
\hline AMAT & 1.75 & 1.04 & 0.08 & 1.06 & 1.75 & 2.45 & 3.47 & $4 \%$ \\
\hline PNC & 0.62 & 0.55 & -0.26 & 0.25 & 0.61 & 0.98 & 1.52 & $13 \%$ \\
\hline $\mathrm{AA}$ & 1.68 & 1.20 & -0.24 & 0.86 & 1.66 & 2.47 & 3.71 & $8 \%$ \\
\hline FMER & 1.46 & 1.07 & -0.23 & 0.73 & 1.41 & 2.14 & 3.31 & $8 \%$ \\
\hline CNQR & 0.60 & 0.73 & -0.58 & 0.09 & 0.59 & 1.08 & 1.80 & $21 \%$ \\
\hline $\mathrm{ROC}$ & 0.17 & 0.29 & -0.30 & -0.02 & 0.17 & 0.37 & 0.66 & $28 \%$ \\
\hline IMGN & 2.07 & 1.86 & -0.90 & 0.78 & 2.00 & 3.30 & 5.22 & $13 \%$ \\
\hline DK & 0.56 & 1.44 & -1.74 & -0.44 & 0.54 & 1.52 & 2.98 & $36 \%$ \\
\hline ARCC & 0.61 & 0.58 & -0.27 & 0.21 & 0.57 & 0.97 & 1.64 & $14 \%$ \\
\hline BXS & 0.74 & 0.98 & -0.78 & 0.06 & 0.70 & 1.36 & 2.45 & $23 \%$ \\
\hline AYI & 0.01 & 0.12 & -0.18 & -0.07 & 0.01 & 0.09 & 0.23 & $48 \%$ \\
\hline
\end{tabular}




\subsubsection{Minor agents with small terminal penalty}

When the terminal penalty for the minor agents is small (on the same order as their temporary impacts), they do not liquidate their inventory by time $\mathrm{T}=1$. As a result, we can see that without any pressure for liquidating inventories, the major agent will simply execute their inventory since the opportunity to recover any prices near time 1 is lost, and the performance gains are affected when using the mean-field game strategy. In particular, the minor HFT agents no longer buy back shares as quickly, so the broker will simply speed up their execution strategy in order to minimize their exposure to the minor agents' impact on their PnL.
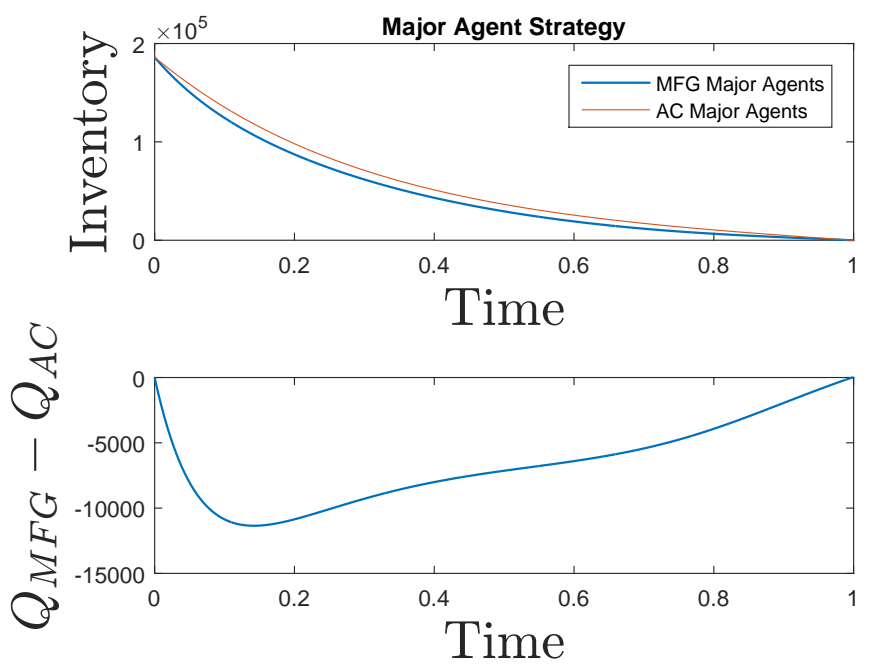

Figure 2.4: Optimal Trading for major agent in the presence of Order Flow and HighFrequency Traders 

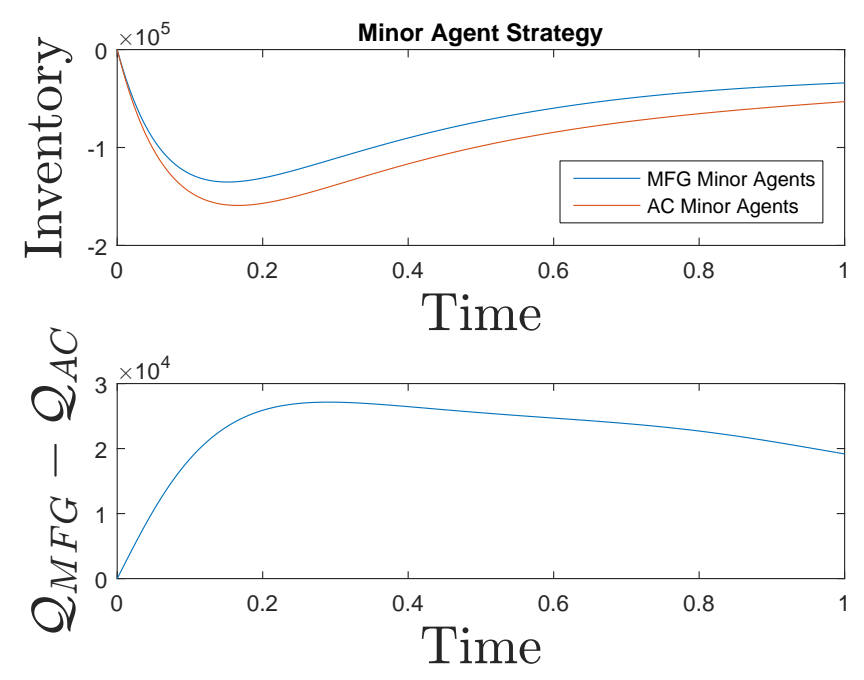

Figure 2.5: Optimal Trading for minor agents

\subsubsection{Impact of Execution Quantity on Performance}

Another factor which affects the performance of the major agent is the amount that she is executing. In Table 2.4 we can see the average increase in performance (again in basis points) for various levels of initial inventory. It should be noted here that the incorporation of noise traders has a larger impact on PnL at small inventory levels, while accounting for high-frequency traders tends to have a more significant impact on $\mathrm{PnL}$ when the inventory size is large.

Generally, the performance of the agent is either strictly increasing with respect to initial inventory, or it is decreasing initially, but increases past a certain inventory level (around 10\%). The trading behaviour is largely consistent with Firoozi and Caines (2016b). 
Table 2.3: Mean-Field vs Almgren-Chriss strategy with small minor agent terminal penalty ( $5 \%$ of Order Flow)

\begin{tabular}{|c|c|c|c|c|c|c|c|c|}
\hline \multirow[b]{2}{*}{ Ticker } & \multirow[b]{2}{*}{ Mean } & \multirow[b]{2}{*}{ StDev } & \multicolumn{5}{|c|}{ Quantiles } & \multirow[b]{2}{*}{$\mathbb{P}(M F G<A C)$} \\
\hline & & & $5 \%$ & $25 \%$ & $50 \%$ & $75 \%$ & $95 \%$ & \\
\hline EBAY & 1.27 & 1.68 & -1.43 & 0.10 & 1.28 & 2.40 & 3.98 & $23 \%$ \\
\hline INTC & 1.94 & 1.87 & -0.94 & 0.70 & 1.83 & 3.19 & 5.09 & $15 \%$ \\
\hline DOW & 1.69 & 2.48 & -2.48 & 0.00 & 1.70 & 3.38 & 5.75 & $25 \%$ \\
\hline $\mathrm{CSCO}$ & 1.46 & 1.57 & -1.13 & 0.39 & 1.47 & 2.54 & 3.98 & $18 \%$ \\
\hline COST & 1.06 & 1.30 & -1.16 & 0.20 & 1.07 & 1.96 & 3.19 & $21 \%$ \\
\hline AXP & 0.70 & 1.73 & -1.98 & -0.42 & 0.66 & 1.89 & 3.52 & $35 \%$ \\
\hline AMAT & 2.17 & 2.63 & -2.32 & 0.40 & 2.15 & 3.89 & 6.43 & $20 \%$ \\
\hline PNC & 0.68 & 1.44 & -1.79 & -0.26 & 0.71 & 1.62 & 3.05 & $30 \%$ \\
\hline $\mathrm{AA}$ & 2.18 & 3.11 & -3.27 & 0.11 & 2.26 & 4.23 & 7.48 & $24 \%$ \\
\hline FMER & 2.16 & 2.82 & -2.95 & 0.45 & 2.25 & 4.12 & 6.66 & $20 \%$ \\
\hline CNQR & 0.73 & 1.99 & -2.51 & -0.59 & 0.72 & 2.03 & 4.11 & $36 \%$ \\
\hline ROC & 0.24 & 0.83 & -1.14 & -0.34 & 0.22 & 0.80 & 1.64 & $39 \%$ \\
\hline IMGN & 2.96 & 5.14 & -5.46 & -0.53 & 2.96 & 6.31 & 11.26 & $28 \%$ \\
\hline DK & 1.10 & 4.04 & -5.18 & -1.51 & 0.99 & 3.69 & 8.22 & $40 \%$ \\
\hline ARCC & 1.19 & 1.57 & -1.38 & 0.16 & 1.27 & 2.25 & 3.64 & $22 \%$ \\
\hline BXS & 1.69 & 2.64 & -2.67 & 0.00 & 1.63 & 3.35 & 6.11 & $25 \%$ \\
\hline AYI & 0.23 & 0.40 & -0.30 & -0.02 & 0.17 & 0.43 & 0.99 & $27 \%$ \\
\hline
\end{tabular}

\subsection{Concluding Remarks}

This paper is the first to generalize the classical optimal liquidation problem to a setting where, in addition to a major agent who is liquidating a large portion of shares, there are a number of minor agents (high-frequency traders (HFTs)) who detect and trade along with the liquidator. Cross interaction between the minor and major agents occur through the impact that each trader has on the drift of the fundamental price. This formulation gives rise to a stochastic dynamic game with mean-field couplings in the fundamental price.

We have shown how this problem can be solved in the mean-field sense resulting in solving a deterministic fixed point problem. Under appropriate conditions, we demonstrated that a unique solution to the fixed point problem exists, and that the resulting decentralized trading strategies form an $\epsilon$-Nash equilibrium for the finite population problem.

The general form of the mean-field optimal strategy is that the minor agents trade along side the major agent, and attempt to gain from the price impact the major agent will have on prices. The major agent anticipates this behaviour and speeds up trading 
Table 2.4: Mean-Field vs Almgren-Criss strategy at various levels of inventory for the major agent

\begin{tabular}{|c|c|c|c|c|c|c|c|c|c|c|}
\hline \multirow[b]{2}{*}{ Ticker } & \multicolumn{10}{|c|}{ Initial Inventory as $\%$ of Order Flow } \\
\hline & $2 \%$ & $4 \%$ & $6 \%$ & $8 \%$ & $10 \%$ & $12 \%$ & $14 \%$ & $16 \%$ & $18 \%$ & $20 \%$ \\
\hline EBAY & 17 & .30 & .41 & 0.54 & 0.69 & 0.83 & 0. & 1.09 & 1.23 & 1.37 \\
\hline & & 38 & & 0.68 & & & & 1.35 & & 1.67 \\
\hline DOV & & 35 & & 64 & 76 & & & 1.22 & & 1.51 \\
\hline $\mathrm{CSCO}$ & & 31 & & 0.52 & & & & 0.98 & & 1.21 \\
\hline & & & & & & & & & & .88 \\
\hline & & & & & & & & & & 0.73 \\
\hline & & & & & & & & & & 1.77 \\
\hline & & & & 0.2 & & & & 0. & & 0.63 \\
\hline & & & & 0.8 & & & & 1. & & 1.74 \\
\hline & & & & 0.9 & & & & 1. & .46 & 1.59 \\
\hline $\mathrm{CNC}$ & & & & 0.39 & & & 0 . & 0.5 & 0.58 & 0.64 \\
\hline & & 16 & & 0.1 & & & & 0 . & 8 & 0.19 \\
\hline IMGN & & 1.71 & & 1.48 & & & & 1.93 & & 2.27 \\
\hline & & & & 0.47 & 0. & 0 & & 0.5 & & 0.66 \\
\hline ARCC & & 0.98 & & 0.70 & 0.66 & & & 0.69 & 73 & 0.75 \\
\hline & & 1.45 & & 0.99 & & & & 0.90 & 0.9 & 0.97 \\
\hline AYI & 0.56 & 0.29 & 0.19 & 0.15 & 0.12 & 0.10 & 0.09 & 0.07 & 0.07 & 0.06 \\
\hline
\end{tabular}

near the beginning and end of the trading horizon to take advantage of the lower price impacts of the minor agents, while slowing down in the middle so that they do not execute too many stocks when the mid-price is low.

There are many directions open for future research, such as including trading constraints for the minor agents and solving for their optimal trading horizon. Another interesting problem would be to solve the problem where the minor agents and major agents do not have distributional information on the initial inventory. Clearly, in real markets agents will be blind to other agent's position, but they may be able to sniff out a large trader liquidating a position. Knowing how to "hide" the major agent's strategy from the minor agents is an important problem that we aim to address in future work. 


\section{Appendix}

\section{A Proof of ODE Derivations}

\section{A.1 Major Agent ODEs}

Here, we follow similar arguments to the ones in Cartea et al. (2015). By employing a standard dynamic programming argument and the use of Itô's formula we obtain the following backward in time Hamilton-Jacobi-Bellman (HJB) equation

$$
\left\{\begin{aligned}
\partial_{t} H_{0}(t, x, f, q, y, u)+\sup _{\nu_{0} \in \mathcal{A}_{0}} \mathcal{L}^{\nu_{0}} H_{0}(t, x, f, q, y, u)-\phi q^{2} & =0, \\
H_{0}(T, x, f, q, y, u) & =x+f q-\alpha q^{2},
\end{aligned}\right.
$$

where $\mathcal{L}^{\nu_{0}}$ is the infinitesimal generator of $\left(\bar{X}(t), \bar{F}_{t}, Q(t)\right)$ satisfying (2.14), and acts as follows

$\mathcal{L}^{\nu_{0}} H_{0}=-\left(f+a_{0} \nu_{0}\right) \nu_{0} \partial_{x} H_{0}+\nu_{0} \partial_{q} H_{0}+\left(\lambda_{0} \nu_{0}+\lambda \bar{\nu}+\lambda_{\mu} \mu_{t}\right) \partial_{f} H_{0}+\bar{\nu} \partial_{y}+\mathcal{L}^{\mu} H_{0}+\zeta H_{0}$.

where $\zeta$ is the operator that encompasses the higher moments of the fundamental price dynamics $f$, and $\mathcal{L}^{\mu}$ is the infinitesimal generator for the noise traders' process $\mu_{t}$ in the HJB equation.

The optimal control in feedback form is

$$
\nu_{0}^{*}:=\arg \sup _{\nu_{0} \in \overline{\mathcal{A}}_{0}} \mathcal{L}^{\nu_{0}} H_{0}=\frac{\left(\lambda_{0} \partial_{f}+\partial_{q}-f \partial_{x}\right) H_{0}}{2 a_{0} \partial_{x} H_{0}} .
$$

which gives

$$
\mathcal{L}^{\nu_{0}^{*}} H_{0}=\frac{\left[\left(\lambda_{0} \partial_{f}+\partial_{q}-f \partial_{x}\right) H_{0}\right]^{2}}{4 a_{0} \partial_{x} H_{0}}+\left(\lambda\left(g_{0}+g_{1} q+g_{2} y+g_{3} u\right)+\lambda_{\mu} \mu_{t}\right) \partial_{f} H_{0}+\bar{\nu} \partial_{y}+\mathcal{L}^{\mu} H_{0}+\zeta H_{0} .
$$


Substitution into the HJB equation (2.42) yields

$$
\begin{aligned}
\left(\partial_{t}+\lambda \bar{\nu}+\lambda_{\mu} u\right. & \left.+\partial_{f}+\bar{\nu} \partial_{y}+\mathcal{L}^{\mu}\right) H_{0}(t, x, f, q, y, u) \\
& +\frac{\left[\left(\lambda_{0} \partial_{f}+\partial_{q}-f \partial_{x}\right) H_{0}(t, x, f, q, y, u)\right]^{2}}{4 a_{0} \partial_{x} H_{0}(t, x, f, q)}-\phi q^{2}=0
\end{aligned}
$$

subject to the terminal condition $H_{0}(T, x, f, q, y, u)=x+f q-\alpha q^{2}$. The terminal condition, and form of this non-linear PDE suggests the ansatz of the form $H_{0}(t, x, f, q)=$ $x+f q+h(t, q, y, u)$ and we find that $h$ solves

$$
\left(\partial_{t}+\bar{\nu} \partial_{y}+\mathcal{L}^{\mu}\right) h(t, q, y, u)+\lambda\left(\bar{\nu}+\lambda_{\mu} u\right) q+\frac{\left(\lambda_{0} q+\partial_{q} h(t, q, y, u)\right)^{2}}{4 a_{0}}-\phi q^{2}=0
$$

with terminal condition $h(T, q, y, u)=-\alpha q^{2}$. Since $q$ appears at most quadratic, we next apply the ansatz $h(t, q, y, u)=h_{0}(t, y, u)+h_{1}(t, y, u) q+h_{2}(t) q^{2}$. Substituting in (2.45) and collecting powers of $q$ yields the coupled system of ODEs following equations (2.25a)-(2.25c).

Substituting this ansatz into (2.43) yields (2.19) for the optimal speed of trading.

Corollary 2.A.1. Assume that the value function $h_{1}(t, y, u)$ for the stochastic optimal control problem (2.19) is a $C^{1}$ function of the variables $t, y$ and $u$. Then, the major agent's auxiliary value functions (2.25b) and (2.25a) admit the ansatz

$$
\begin{aligned}
& h_{0}(t, y, u)=h_{0}^{0}(t)+h_{0}^{1}(t) y+h_{0}^{2}(t) y^{2}+h_{0}^{\mu}(t) u y+h_{0}^{0, \mu} u+h_{0}^{2, \mu} u^{2} \\
& h_{1}(t, y, u)=h_{1}^{0}(t)+h_{1}^{1}(t) y+h_{1}^{\mu}(t) u
\end{aligned}
$$


and the system of PDEs above reduce to the following system of ODEs

$$
\begin{aligned}
\partial_{t} h_{0}^{0}+g_{0} h_{0}^{1}+\frac{1}{4 a_{0}}\left(h_{1}^{0}\right)^{2}=0, & h_{0}^{0}(T)=0, \\
\partial_{t} h_{0}^{1}+2 g_{0} h_{0}^{2}+g_{2} h_{0}^{1}+\frac{1}{2 a_{0}} h_{1}^{0} h_{1}^{1}=0, & h_{0}^{1}(T)=0, \\
\partial_{t} h_{0}^{2}+2 g_{2} h_{0}^{2}+\frac{1}{4 a_{0}}\left(h_{1}^{1}\right)^{2}=0, & h_{0}^{2}(T)=0, \\
\left(\partial_{t}-\kappa\right) h_{0}^{\mu}+2 g_{3} h_{0}^{2}+\frac{1}{2 a_{0}} h_{1}^{\mu} h_{1}^{1}=0, & h_{0}^{\mu}(T)=0, \\
\left(\partial_{t}+\lambda g_{3} h_{0}^{\mu}-\kappa\right) h_{0}^{0, \mu}+\frac{1}{2 a_{0}} h_{1}^{0} h_{1}^{\mu}=0, & h_{0}^{0, \mu}(T)=0, \\
\left(\partial_{t}-\kappa\right) h_{0}^{2, \mu}+\frac{1}{2 a_{0}} h_{1}^{1} h_{1}^{\mu}=0, & h_{0}^{2, \mu}(T)=0, \\
\partial_{t} h_{1}^{0}+g_{0} h_{1}^{1}+h_{0}^{1} g_{1}+\lambda g_{0}+\frac{1}{2 a_{0}}\left(\lambda_{0}+2 h_{2}\right) h_{1}^{0}=0, & h_{1}^{0}(T)=0, \\
\partial_{t} h_{1}^{1}+2 h_{0}^{2} g_{1}+\lambda g_{2}+\frac{1}{2 a_{0}}\left(\lambda_{0}+2 h_{2}+2 a_{0} g_{2}\right) h_{1}^{1}=0, & h_{1}^{1}(T)=0, \\
\left(\partial_{t}-\kappa\right) h_{1}^{\mu}+g_{3} h_{1}^{1}+\left(\lambda g_{3}+\lambda_{\mu}\right)+\frac{1}{2 a_{0}}\left(\lambda_{0}+2 h_{2}\right) h_{1}^{\mu}=0, & h_{1}^{\mu}(T)=0 .
\end{aligned}
$$

\section{A.2 Minor Agent ODEs}

Here, we follow similar arguments to the ones in Cartea et al. (2015). By employing a standard dynamic programming argument and the use of Itô's formula we get the following backward in time HJB equation

$$
\left\{\begin{aligned}
\partial_{t} H_{i}\left(t, x, f, q_{i}, q, y, u\right)+\sup _{\nu_{i} \in \overline{\mathcal{A}}_{i}} \mathcal{L}^{\nu_{i}} H_{i}\left(t, x, f, q_{i}, q, y, u\right) & =0, \\
H_{i}\left(T, x, f, q_{i}, q\right) & =x+f q_{i}-\psi q_{i}^{2},
\end{aligned}\right.
$$

where $\mathcal{L}^{\nu_{i}}$ is the infinitesimal generator of $\left(\bar{X}(t), \bar{F}_{t}, Q(t), \mathcal{Q}_{t}, \mu_{t}\right)$ satisfying (2.15), and acts as follows

$\mathcal{L}^{\nu_{i}} H_{i}=\left[-\left(f+a \nu_{i}\right) \nu_{i} \partial_{x}+\nu_{i} \partial_{q_{i}}+\left(\lambda_{0} \nu_{0}^{*}+\lambda \bar{\nu}+\lambda_{\mu} \mu_{t}\right) \partial_{f}+\nu_{0} \partial_{q}+\bar{\nu} \partial_{y}+\mathcal{L}^{\mu} H_{i}+\zeta\right] H_{i}$.

The optimal control in feedback form is

$$
\nu_{i}^{*}:=\arg \sup _{\nu_{i} \in \overline{\mathcal{A}}_{i}} \mathcal{L}^{\nu_{i}} H_{i}=\frac{\left(\partial_{q}-f \partial_{x}\right) H_{i}}{2 a \partial_{x} H_{i}}
$$


which gives

$\mathcal{L}^{\nu_{i}^{*}} H_{i}=\frac{\left[\left(\partial_{q}-f \partial_{x}\right) H_{i}\right]^{2}}{4 a \partial_{x} H_{i}}+\left(\lambda_{0} \nu_{0}^{*}+\lambda \bar{\nu}+\lambda_{\mu} \mu_{t}\right) \partial_{f} H_{i}+\mathcal{L}^{\mu} H_{i}+\nu_{0} \partial_{q} H_{i}+\bar{\nu} \partial_{y} H_{i}+\zeta H_{i}$.

Substituting into the HJB equation (2.47) yields

$\partial_{t} H_{i}+\frac{\left[\left(\partial_{q}-f \partial_{x}\right) H_{i}\right]^{2}}{4 a \partial_{x} H_{i}}+\left(\lambda_{0} \nu_{0}^{*}+\lambda \bar{\nu}+\lambda_{\mu} \mu_{t}\right) \partial_{f} H_{i}+\mathcal{L}^{\mu} H_{i}+\nu_{0} \partial_{q} H_{i}+\bar{\nu} \partial_{y} H_{i}+\zeta H_{i}=0$

with terminal condition $H_{i}\left(T, x, f, q_{i}, q, y, u\right)=x+f q_{i}-\psi q_{i}^{2}$. We next separate the book value of the cash and assets in hand from value of optimally trading the remaining shares by applying the ansatz $H_{i}\left(t, x, f, q_{i}, q\right)=x+f q_{i}+l\left(t, q_{i}, q, y, u\right)$ where $h$ solves the PDE

$$
\partial_{t} l+\frac{1}{4 a}\left(\partial_{q_{i}} l\right)^{2}+q\left(\lambda_{0} \nu_{0}^{*}+\lambda \bar{\nu}+\lambda_{\mu} \mu_{t}\right)+\mathcal{L}^{\mu} l+\nu_{0} \partial_{q} l+\bar{\nu} \partial_{y} l=0
$$

with terminal condition $l\left(T, q_{i}, q, y, u\right)=-\psi q_{i}^{2}$. Next, noticing that the terminal condition and the PDE is at most quadratic in $q$, we apply a further ansatz $l\left(t, q_{i}, q, y, u\right)=$ $l_{0}(t, q, y, u)+l_{1}(t, q, y, u) q_{i}+l_{2}(t) q_{i}^{2}$ to (2.49) yields the following coupled system of PDEs

$$
\begin{array}{rr}
\left(\partial_{t}+\nu_{0} \partial_{q}+\bar{\nu} \partial_{y}+\mathcal{L}^{\mu}\right) l_{0}(t)+\frac{1}{4 a} l_{1}^{2}(t)=0, & l_{0}(T, y, u)=0 \\
\left(\partial_{t}+\nu_{0} \partial_{q}+\bar{\nu} \partial_{y}+\mathcal{L}^{\mu}\right) l_{1}(t)+\frac{1}{a}\left(l_{1}(t) l_{2}(t)\right)+\left(\lambda_{0} \nu_{0}+\lambda \bar{\nu}\right) & (2.50 \mathrm{~b}) \\
\partial_{t} l_{2}(t)+\frac{1}{a}\left(l_{2}(t)\right)^{2}=0, & l_{1}(T, y, u)=0 \\
l_{2}(T)=-\psi .
\end{array}
$$

where we denote

$$
\nu_{0}=\nu_{0}(t, q, y, u) \quad \bar{\nu}=\bar{\nu}(t, q, y, u)
$$

Finally, substituting the sequence of ansatz into the feedback form for the optimal control in (2.48) leads directly to (2.21).

Corollary 2.A.2. Assume that the value function $H_{i}(t, x, f, q)$ for the auxiliary stochastic optimal control problem (2.15) is a $C^{1}$ function of the variables $t, x$ and $q$, and $C^{2}$ function of variable $f$. Then, a generic minor agent's auxiliary value function (2.50b) 
admits the ansatz

$$
l_{1}(t, q, y, u)=l_{1}^{0}(t)+l_{1}^{1}(t) q+l_{1}^{2}(t) y+l_{1}^{\mu}(t)
$$

which results in the set of $P D E s$

$$
\begin{array}{rr}
\partial_{t} l_{1}^{0}(t)+g_{0}(t) l_{1}^{2}(t)+\frac{h_{1}^{0}(t)}{2 a_{0}}\left(\lambda_{0}+l_{1}^{0}(t)\right)+\frac{1}{a}\left(l_{1}^{0}(t) l_{2}(t)\right)+\lambda g_{0}(t)=0, & l_{1}^{0}(T)=0 \\
\partial_{t} l_{1}^{1}(t)+\frac{1}{a}\left(l_{1}^{1}(t) l_{2}(t)\right)+g_{1}(t)\left(\lambda+l_{1}^{2}(t)\right)+\frac{h_{1}^{1}(t)}{2 a_{0}}\left(l_{1}^{1}(t)+\lambda_{0}\right)=0, & l_{1}^{1}(T)=0 \\
\partial_{t} l_{1}^{2}(t)+\frac{1}{a}\left(l_{1}^{2}(t) l_{2}(t)\right)+g_{2}(t) l_{1}^{2}(t)+\frac{\lambda_{0}+2 h_{2}}{2 a_{0}}\left(\lambda_{0}+l_{1}^{2}(t)\right)+\left(\lambda g_{2}(t)\right)=0, & l_{1}^{2}(T)=0 \\
\left(\partial_{t}-\kappa\right)\left(l_{1}^{\mu}(t)+g_{3}(t)\right) l_{1}^{2}(t)+\frac{h_{1}^{\mu}(t)}{2 a_{0}} l_{1}^{\mu}(t)+\frac{1}{a} l_{1}^{\mu}(t) l_{2}(t)+\lambda g_{3}(t)+\lambda_{\mu}=0, & (2.51 \mathrm{c}) \\
& l_{1}^{\mu}(T)=0
\end{array}
$$

\section{B Proof of $\epsilon$-Nash Equilibrium}

\section{B.1 Proof of 2.5.1}

First, the triangle inequality implies that

$$
\mathbb{E}\left|F_{-i}^{*}(t)-\bar{F}^{*}(t)\right| \leq \mathbb{E}\left|F_{-i}^{*}(t)-F^{*}(t)\right|+\mathbb{E}\left|F^{*}(t)-\bar{F}^{*}(t)\right|
$$

where $F^{*}(\cdot)$ is given in (2.28). From $(2.28),(2.31)$ and $(2.37)$ we respectively get

$$
\begin{aligned}
& F^{*}(t)=F_{0}+\int_{0}^{t}\left(\lambda_{0} \nu_{0}(t, q, y)+\frac{\lambda}{N} \sum_{i=1}^{N} \nu_{i}\left(t, q_{i}, q, y\right)\right) d t+M_{t} \\
& \bar{F}^{*}(t)=F_{0}+\int_{0}^{t}\left(\lambda_{0} \nu_{0}^{*}(t, q, y)+\lambda\left(g_{0}(t)+g_{1}(t) Q_{0}+g_{2}(t) \mathcal{Q}\right)\right) d t+M_{t} \\
& F_{-i}^{*}(t)=F_{0}+\int_{0}^{t}\left(\lambda_{0} \nu_{0}^{*}(t, q, y)+\frac{\lambda}{N} \sum_{j=1, j \neq i}^{N} \nu_{j}^{*}\left(t, q_{j}, q, y\right)+\frac{\lambda}{N} \nu_{i}\left(t, q_{i}, q, y\right)\right) d t+M_{t} .
\end{aligned}
$$


Therefore, we have

$$
\mathbb{E}\left|F_{-i}^{*}(t)-F^{*}(t)\right|=\mathbb{E}\left|\frac{\lambda}{N} \int_{0}^{t}\left(\nu_{0, t}+\sum_{j=1}^{N} \nu_{j}(t)-\sum_{j=1}^{N} \nu_{j}^{*}(t)-\tilde{\nu}_{0}^{*}(t)\right) d t\right|=O\left(\frac{1}{N}\right),
$$

since $\sup _{0 \leq t \leq T}\left|\nu_{i}^{*}(t)-\nu_{i}(t)\right|<\infty$. We also have

$$
\mathbb{E}\left|F^{*}(t)-\bar{F}^{*}(t)\right|=\mathbb{E}\left|\lambda \int_{0}^{t}\left(\frac{1}{N} \sum_{i=1}^{N} \nu_{i}^{*}\left(t, q_{i}, q, y\right)-\bar{\nu}^{*}(t, q, y)\right)\right|
$$

using (2.26b). We have that

$$
\mathbb{E}\left|\frac{1}{N} \sum_{i=1}^{N} Q_{i, t}-\mathcal{Q}_{t}\right|=\mathbb{E}\left|\int_{0}^{t}\left(\frac{1}{N} \sum_{i=1}^{N} \nu_{i}^{*}\left(t, q_{i}, q, y\right)-\bar{\nu}^{*}(t, q, y)\right)\right|
$$

so that by Gronwall's Lemma, we have that there exists a constant $C \in R^{+}$such that for all $t \in[0, T]$,

$$
\begin{aligned}
\mathbb{E}\left|\frac{1}{N} \sum_{i=1}^{N} Q_{i, t}-\mathcal{Q}_{t}\right| & =\mathbb{E}\left|\int_{0}^{t}\left(-\frac{1}{\frac{a}{\psi}+(T-t)}+\frac{1}{2 a} l_{1}^{2}(t)\right)\left(\frac{1}{N} \sum_{i=1}^{N} Q_{i, t}-\mathcal{Q}_{t}\right)\right| \\
& \leq C\left|\frac{1}{N} \sum_{i=1}^{N} Q_{i, 0}-\mathcal{Q}_{0}\right|
\end{aligned}
$$

Therefore, the trading speed has the representation

$$
\begin{aligned}
\mathbb{E}\left|F^{*}(t)-\bar{F}^{*}(t)\right| & =\lambda \mathbb{E}\left|\int_{0}^{t}\left(\frac{1}{N} \sum_{i=1}^{N} \nu_{i}^{*}\left(t, q_{i}, q, y\right)-\bar{\nu}^{*}(t, q, y)\right)\right| \\
& \leq \lambda C T \epsilon_{N}
\end{aligned}
$$

where $\epsilon_{N}$ is defined in (2.29). Hence, this together with (2.52) and (2.53) implies that

$$
\mathbb{E}\left|F_{-i}^{*}(t)-\bar{F}^{*}(t)\right|=O\left(\epsilon_{N}\right)+O\left(\frac{1}{N}\right), \quad 0 \leq t \leq T .
$$

Next, from (2.30) and (2.39) we have

$$
\begin{aligned}
& \bar{X}_{-i}^{*}(t)=X_{i, 0}-\int_{0}^{t}\left(\bar{F}^{*}(t)+a \nu_{i}\left(t, q_{i}, q, y\right)\right) \nu_{i}\left(t, q_{i}, q, y\right) d t \\
& X_{-i}^{*}(t)=X_{i, 0}-\int_{0}^{t}\left(F_{-i}^{*}(t)+a \nu_{i}\left(t, q_{i}, q, y\right)\right) \nu_{i}\left(t, q_{i}, q, y\right) d t .
\end{aligned}
$$


Therefore,

$$
\begin{aligned}
\mathbb{E}\left|X_{-i}^{*}(t)-\bar{X}_{-i}^{*}(t)\right| & =\mathbb{E}\left|\int_{0}^{t}\left(\bar{F}^{*}(t)-F_{-i}^{*}(t)\right) \nu_{i}\left(t, q_{i}, q, y\right) d t\right| \\
& \leq T \sup _{0 \leq t \leq T}\left|v_{i}\left(t, q_{i}, q, y\right)\right|\left(\lambda C^{\prime} \epsilon_{N}+O\left(\frac{1}{N}\right)\right)
\end{aligned}
$$

from (2.53) and (2.55), where we recall that $C^{\prime}=\lambda T \sup _{0 \leq t \leq T}\left|\nu_{i}\left(t, q_{i}, q, y\right)-\nu_{i}^{*}\left(t, q_{i}, q, y\right)\right|<$ $\infty$ is independent of $N$. This implies that

$$
\mathbb{E}\left|X_{-i}^{*}(t)-\bar{X}_{-i}(t)\right|=O\left(\epsilon_{N}\right)+O\left(\frac{1}{N}\right) .
$$

On the other hand, from (2.34) and (2.36) we respectively get

$$
\begin{aligned}
& \bar{F}_{-0}^{*}(t)=F_{0}+\int_{0}^{t}\left(\lambda_{0} \nu_{0}(t, q, y)+\left(g_{0}(t)+g_{1}(t) q+g_{2}(t) y\right)\right) d t+M_{t} \\
& F_{-0}^{*}(t)=F_{0}+\int_{0}^{t}\left(\lambda_{0} \nu_{0}(t, q, y)+\frac{\lambda}{N} \sum_{i=1}^{N} \nu_{i}^{*}\left(t, q_{i}, q, y\right)\right) d t+M_{t} .
\end{aligned}
$$

Following arguments exactly parallel to those used in proving (2.55), we get

$$
\mathbb{E}\left|F_{-0}^{*}(t)-\bar{F}_{-0}^{*}(t)\right| \leq \lambda C^{\prime} \epsilon_{N}
$$

where $\epsilon_{N}$ is defined in (2.29). Next, from (2.35) and (2.38) we have

$$
\begin{aligned}
& \bar{X}_{-0}^{*}(t)=X_{0,0}-\int_{0}^{t}\left(\bar{F}_{-0}^{*}(t)+a_{0} \nu_{0}(t, q, y)\right) \nu_{0}(t, q, y) d t \\
& X_{-0}^{*}(t)=X_{0,0}-\int_{0}^{t}\left(F_{-0}^{*}(t)+a_{0} \nu_{0}(t, q, y)\right) \nu_{0}(t, q, y) d t .
\end{aligned}
$$

Therefore,

$$
\begin{aligned}
\mathbb{E}\left|X_{-0}^{*}(t)-\bar{X}_{-0}^{*}(t)\right| & =\mathbb{E}\left|\int_{0}^{t}\left(\bar{F}_{-0}^{*}(t)-F_{-0}^{*}(t)\right) \nu_{0}(t, q, y) d t\right| \\
& \leq \lambda T \sup _{0 \leq t \leq T}\left|v_{0}(t, q, y)\right| C^{\prime} \epsilon_{N}
\end{aligned}
$$

from (2.57). This together with (2.57) concludes the proof. 


\section{B.2 Proof of 2.40}

For this proof, the fact that $\nu_{0}, \nu_{i}$ are functions of $q_{i}, q, y$ are purposely omitted for clarity. The left inequality is trivial. To establish the right inequality, we consider following two cases separetely.

Case $I$. Let us assume that a representative minor agent $1 \leq i \leq N$ uses an alternative strategy $\nu_{i} \in \mathcal{A}_{i}$ while all other agents apply their mean-field game control laws. Then, the wealth function of the minor agent defined in (2.10) will be of the form

$$
J_{i}\left(\nu_{i} ; \nu_{-i}^{*}\right)=\mathbb{E}\left[X_{-i}^{*}(T)+Q_{i, t}\left(F_{-i}^{*}(T)-\psi Q_{i, t}\right)\right]
$$

where the dynamics of $F_{-i}^{*}(\cdot)$ and $X_{-i}^{*}(\cdot)$ are given in (2.37) and (2.39), respectively. We make the decomposition

$$
\begin{gathered}
J_{i}\left(\nu_{i} ; \nu_{-i}^{*}\right)=\mathbb{E}\left[\bar{X}_{-i}^{*}(T)+Q_{i, t}\left(\bar{F}^{*}(T)-\psi Q_{i, t}\right)+\left(X_{-i}^{*}(T)-\bar{X}_{-i}^{*}(T)\right)\right. \\
\left.+Q_{i, t}\left(F_{-i}^{*}(T)-\bar{F}^{*}(T)\right)\right]
\end{gathered}
$$

where $\bar{X}_{-i}^{*}(\cdot)$ and $\bar{F}^{*}(\cdot)$ are given in (2.30) and (2.31), respectively. The linearity of the expectation operator implies that

$$
\begin{aligned}
J_{i}\left(\nu_{i} ; \nu_{-i}^{*}\right)= & \mathbb{E}\left[\bar{X}_{-i}^{*}(T)+Q_{i, t}\left(\bar{F}^{*}(T)-\psi Q_{i, t}\right)\right]+\mathbb{E}\left[X_{-i}^{*}(T)-\bar{X}_{-i}^{*}(T)\right] \\
& +\mathbb{E}\left[Q_{i, t}\left(F_{-i}^{*}(T)-\bar{F}^{*}(T)\right)\right]=: \bar{J}_{i}\left(\nu_{i}\right)+I_{1}+I_{2}
\end{aligned}
$$

where $\bar{J}_{i}\left(\nu_{i}\right)$ is the utility function of the auxiliary stochastic optimal control problem of the generic minor agent defined in (2.15). But, $\nu_{i}^{*}(\cdot) \in \mathcal{A}_{i}$ is the optimal control with respect to the utility function $\bar{J}_{i}\left(\nu_{i}\right)$, i.e. $\nu_{i}^{*}=\arg \sup _{\nu_{i} \in \mathcal{A}_{i}} \bar{J}_{i}\left(\nu_{i}\right)$, by the construction of the mean-field game system (2.23) in Section 2.4.1. Therefore, we have

$$
\begin{aligned}
& \bar{J}_{i}\left(\nu_{i}\right) \leq \bar{J}_{i}\left(\nu_{i}^{*}\right), \\
& I_{1}=O\left(\epsilon_{N}\right)+O\left(\frac{1}{N}\right),
\end{aligned}
$$

where (2.63) follows from Lemma 2.5.1. Moreover, the Cauchy-Schwarz inequality gives

$$
\left|I_{2}\right| \equiv\left|\mathbb{E}\left[Q_{i, t}\left(F_{-i}^{*}(T)-\bar{F}^{*}(T)\right)\right]\right| \leq\left(\mathbb{E}\left|Q_{i, t}\right|^{2}\right)^{1 / 2} \times\left(\mathbb{E}\left|F_{-i}^{*}(T)-\bar{F}^{*}(T)\right|^{2}\right)^{1 / 2} .
$$


Using the inequality $(x+y)^{2} \leq 2 x^{2}+2 y^{2}$, one can show that

$$
\left(\mathbb{E}\left|F_{-i}^{*}(T)-\bar{F}^{*}(T)\right|^{2}\right)^{1 / 2}=O\left(\epsilon_{N}\right)+O\left(\frac{1}{N}\right)
$$

by following the same argument as in proving (2.56) in Lemma 2.5.1. On the other hand, by the Cauchy-Schwarz inequality that $\left|\int_{0}^{T} F_{t} d t\right|^{2} \leq T \int_{0}^{T}\left|F_{t}\right|^{2} d t$ we have

$$
\begin{aligned}
\mathbb{E}\left|Q_{i, t}\right|^{2}= & \mathbb{E}\left|Q_{i, 0}+\int_{0}^{T} \nu_{i, t} d t\right|^{2} \\
& \leq 2 \mathbb{E}\left|Q_{i, 0}\right|^{2}+2 T \int_{0}^{T}\left|v_{i}(t)\right|^{2} d t \leq 2 k+2 T^{2} \sup _{0 \leq t \leq T}\left|v_{i}(t)\right|^{2}<\infty
\end{aligned}
$$

where $k$ is defined in (A1). This together with (2.64) and (2.65) gives

$$
I_{2}=O\left(\epsilon_{N}\right)+O\left(\frac{1}{N}\right)
$$

From (2.61)-(2.63) and (2.66) we get

$$
J_{i}\left(\nu_{i} ; \nu_{-i}^{*}\right)=\bar{J}_{i}\left(\nu_{i}\right)+I_{1}+I_{2} \leq \bar{J}_{i}\left(\nu_{i}^{*}\right)+O\left(\epsilon_{N}\right)+O\left(\frac{1}{N}\right) .
$$

On the other hand, we have

$$
J_{i}\left(\nu_{i}^{*} ; \nu_{-i}^{*}\right)=\mathbb{E}\left[X_{i}^{*}(T)+Q_{i}^{*}(T)\left(F^{*}(T)-\psi Q_{i}^{*}(T)\right)\right],
$$

where $X_{i}^{*}(\cdot), Q_{i}^{*}(\cdot)$ and $F^{*}(\cdot)$ are given in (2.27)-(2.28). As in (2.60) we make the decomposition

$$
\begin{aligned}
J_{i}\left(\nu_{i}^{*} ; \nu_{-i}^{*}\right)=\mathbb{E}\left[\bar{X}_{i}^{*}(T)+Q_{i}^{*}(T)\left(\bar{F}^{*}(T)-\psi Q_{i}^{*}(T)\right)+\left(X_{i}^{*}(T)-\bar{X}_{i}^{*}(T)\right)\right. \\
\left.\quad+Q_{i}^{*}(T)\left(F^{*}(T)-\bar{F}^{*}(T)\right)\right] \\
=\bar{J}_{i}\left(\nu_{i}^{*}\right)+\mathbb{E}\left[X_{i}^{*}(T)-\bar{X}_{i}^{*}(T)\right]+\mathbb{E}\left[Q_{i}^{*}(T)\left(F^{*}(t)-\bar{F}^{*}(t)\right)\right],
\end{aligned}
$$

where $\bar{F}^{*}(\cdot)$ and $\bar{X}_{i}^{*}(\cdot)$ are defined in (2.31) and (2.33), respectively, and we used the linear property of the expectation operator in the last equality. Following the same argument in the proof of Lemma 2.56, and in (2.63) and (2.66) above we can show that

$$
\left|\mathbb{E}\left[X_{i}^{*}(T)-\bar{X}_{i}^{*}(T)\right]+\mathbb{E}\left[Q_{i}^{*}(T)\left(F^{*}(t)-\bar{F}^{*}(t)\right)\right]\right|=O\left(\epsilon_{N}\right) .
$$


This together with (2.68) gives

$$
\left|J_{i}\left(\nu_{i}^{*} ; \nu_{-i}^{*}\right)-\bar{J}_{i}\left(\nu_{i}^{*}\right)\right|=O\left(\epsilon_{N}\right) .
$$

Finally, (2.67) and (2.69) imply that

$$
J_{i}\left(\nu_{i} ; \nu_{-i}^{*}\right) \leq J_{i}\left(\nu_{i}^{*} ; \nu_{-i}^{*}\right)+O\left(\epsilon_{N}\right)+O\left(\frac{1}{N}\right)
$$

which concludes the right inequality (2.40) for the representative minor agent $i$.

Case II. Let us assume that the major agent uses an alternative strategy $\nu_{0} \in \mathcal{A}_{0}$ while all minor agents apply the mean-field game trading laws. Then, we may write the wealth function of the major agent defined in (2.9) as

$$
J_{0}\left(\nu_{0} ; \nu_{-0}^{*}\right)=\mathbb{E}\left[X_{-0}^{*}(T)+Q_{0, T}\left(F_{-0}^{*}(T)-\psi Q_{0, T}\right)-\phi \int_{0}^{T}\left(Q_{0}(u)\right)^{2} d u\right]
$$

where $F_{-0}^{*}(\cdot)$ and $X_{-0}^{*}(\cdot)$ are given in $(2.36)$ and $(2.38)$, respectively. We make the decomposition

$$
\begin{aligned}
J_{0}\left(\nu_{0} ; \nu_{-0}^{*}\right)= & \mathbb{E}\left[\bar{X}_{-0}^{*}(T)+Q_{0, T}\left(\bar{F}_{-0}^{*}(T)-\psi Q_{0, T}\right)-\phi \int_{0}^{T}\left(Q_{0}(u)\right)^{2} d u\right. \\
& \left.+\left(X_{-0}^{*}(T)-\bar{X}_{-0}^{*}(T)\right)+Q_{0, T}\left(F_{-0}^{*}(t)-\bar{F}_{-0}^{*}(t)\right)\right] \\
= & \bar{J}_{0}\left(\nu_{0}\right)+\mathbb{E}\left[X_{-0}^{*}(T)-\bar{X}_{-0}^{*}(T)\right]+\mathbb{E}\left[Q_{0, T}\left(F_{-0}^{*}(t)-\bar{F}_{-0}^{*}(t)\right)\right] \\
= & : \bar{J}_{0}\left(\nu_{0}\right)+L_{1}+L_{2} .
\end{aligned}
$$

where $\bar{F}_{-0}^{*}(\cdot)$ and $\bar{X}_{-0}^{*}(\cdot)$ are given in $(2.34)$ and $(2.35)$, respectively, and $\bar{J}_{0}\left(\nu_{0}\right)$ is the utility function of the auxiliary stochastic optimal control problem of the major agent defined in (2.14). But, $\nu_{0}^{*}(\cdot) \in \mathcal{A}_{0}$ is the optimal control with respect to the utility function $\bar{J}_{0}\left(\nu_{0}\right)$, i.e. $\nu_{0}^{*}=\arg \sup _{\nu_{0} \in \mathcal{A}_{0}} \bar{J}_{0}\left(\nu_{0}\right)$, by the construction of the mean-field game system (2.23) in Section 2.4.1. We note that the postulated mean-field form (2.13) let us get the result that $\nu_{0}^{*}=\arg \sup _{\nu_{0} \in \mathcal{A}_{0}} \bar{J}_{0}\left(\nu_{0}\right)$ above, regardless the difference between $\bar{F}(\cdot)$ given in (2.14) and $\bar{F}_{-0}^{*}(\cdot)$ in (2.34).

Following arguments exactly parallel to those used in Case $I$ above yield

$$
\begin{aligned}
& L_{1}+L_{2}=O\left(\epsilon_{N}\right), \\
& \left|J_{0}\left(\nu_{0}^{*} ; \nu_{-0}^{*}\right)-\bar{J}_{0}\left(\nu_{0}^{*}\right)\right|=O\left(\epsilon_{N}\right) .
\end{aligned}
$$


Finally, (2.71)-(2.73) imply that

$$
J_{0}\left(\nu_{0} ; \nu_{-0}^{*}\right) \leq J_{0}\left(\nu_{0}^{*} ; \nu_{-0}^{*}\right)+O\left(\epsilon_{N}\right)
$$

which concludes the right inequality (2.40) for the major agent. This completes the proof. 


\section{C Simulation parameters}

In our simulations, we assigned the following parameters based on our estimated parameters

$$
\lambda_{0}=\lambda=\hat{b} \quad a_{0}=10 \cdot a=\hat{k}
$$

and $\lambda_{M O}^{ \pm}$is the rate of arrival of market orders, and $\mathbb{E}\left[\eta^{ \pm}\right]$is the jump size that is incurred at that time by noise traders. The following parameters were estimated in Cartea and Jaimungal (2016).

Table 2.C.1: Simulation Parameters

\begin{tabular}{rrrrrrrrr}
\hline \hline Ticker & Mid-Price & $\sigma$ & $\hat{b}\left(\times 10^{-6}\right)$ & $\hat{k}\left(\times 10^{-6}\right)$ & $\lambda_{M O}^{+}$ & $\lambda_{M O}^{-}$ & $\mathbb{E}\left[\eta^{+}\right]$ & $\mathbb{E}\left[\eta^{-}\right]$ \\
\hline EBAY & 53.41 & 0.17 & 4.49 & 2.50 & 549.41 & 585.13 & 358.36 & 347.18 \\
INTC & 30.26 & 0.16 & 1.09 & 0.44 & 438.86 & 453.91 & $1,048.99$ & $1,013.83$ \\
DOW & 49.48 & 0.23 & 6.87 & 3.01 & 389.76 & 400.67 & 282.28 & 286.41 \\
CSCO & 24.21 & 0.14 & 0.63 & 0.24 & 306.70 & 307.99 & $1,500.35$ & $1,376.49$ \\
COST & 121.37 & 0.12 & 25.30 & 10.60 & 294.98 & 293.71 & 139.89 & 141.38 \\
AXP & 89.78 & 0.19 & 17.20 & 8.95 & 281.10 & 273.04 & 159.27 & 158.29 \\
AMAT & 20.85 & 0.23 & 1.60 & 0.60 & 248.86 & 247.66 & 861.38 & 833.01 \\
PNC & 84.67 & 0.15 & 19.90 & 10.00 & 206.79 & 209.22 & 136.35 & 143.73 \\
AA & 14.44 & 0.27 & 1.49 & 0.59 & 154.65 & 152.89 & 953.22 & 990.34 \\
FMER & 19.17 & 0.21 & 8.74 & 3.14 & 104.04 & 105.58 & 254.01 & 247.77 \\
CNQR & 105.96 & 0.29 & 88.60 & 76.20 & 96.47 & 99.96 & 128.79 & 136.23 \\
ROC & 76.10 & 0.19 & 43.40 & 56.10 & 96.00 & 93.21 & 114.76 & 117.90 \\
IMGN & 12.19 & 0.50 & 15.90 & 9.97 & 90.61 & 87.21 & 218.09 & 221.55 \\
DK & 30.83 & 0.78 & 45.50 & 48.30 & 80.26 & 86.34 & 118.25 & 117.12 \\
ARCC & 16.96 & 0.12 & 2.83 & 1.32 & 54.83 & 55.46 & 583.79 & 545.10 \\
BXS & 22.88 & 0.26 & 27.20 & 17.20 & 47.64 & 48.19 & 143.36 & 143.23 \\
AYI & 128.21 & 0.31 & 203.00 & 656.00 & 41.57 & 40.66 & 106.51 & 107.41 \\
\hline \hline
\end{tabular}




\section{Chapter 3}

\section{Robust Stochastic Games and Systemic Risk}

In the previous section, we introduced a MFG model with ambiguity aversion for a trader whom wishes to maximize her profits. Another setting in which game theory naturally arises is bank reserves. A bank would want to track the average reserve of other banks, as having a relatively high reserve would mean they lose revenue on interest, and a relatively low reserve would increase their chance of default. In addition, there are costs associated with moving reserves to and from the central bank.

This setup is further extended with the notion of systemic risk, which can cause multiple banks to exceed a default threshold within a given timeframe. This framework has been looked at by Carmona et al. (2015) in the absence of ambiguity aversion.

\subsubsection{Systemic Risk}

In contrast to the previous chapter, this chapter will derive the optimal control as well as the dynamics through the Pontryagin Maximum Principle, rather than the HamiltonJacobi-Bellman equation. Given the common noise that we will introduce in the dynamics, the HJB approach would rely on a verification theorem for a stochastic PDE, which require very strong assumptions and generally rely on connections between the Pontryagin maximum principle and the HJB equation. In the appendix to this chapter, we prove a Nash-Pontryagin verification theorem directly under a convex-concave assumption. Interbank lending is one of the aspects of financial institutions which are not collatoralized, or insured against. As a result, banks tend to be more sensitive to each others' financial stability than their balance sheets may suggest. A more stable source for borrowing is the central bank, but it usually comes at a higher rate. We first consider the 
setup of Carmona et al. (2015) in which they look at the optimal rate which banks should borrow from the central bank. We then extend this setup to include ambiguity aversion, and then look at the impact of the resulting control to the bank's default probability. We refer the reader to Freixas et al. (2000), Acharya et al. (2017), Haldane and May (2011), Rochet and Tirole (1996) for further discussion of sources of systemic risk and methods for measuring a financial institution's contribution to systemic risk.

\subsection{Introduction}

Recently, Carmona et al. (2015) investigated interbank borrowing and lending with systemic risk in a stochastic game setting. The authors propose a tractable linear-quadratic game for the dynamics of bank's log-reserves. They are successful at providing closed form solutions for the stochastic game both in the finite and infinite player limit (where they study the problem from a mean-field game perspective). Moreover, they demonstrate default risk can be reduced when the system is viewed as stochastic game, rather than each firm optimizing independently. Other related studies of interbank borrowing and lending include Fouque and Ichiba (2013), Fouque and Langsam (2013), Bo and Capponi (2015) and Biagini et al. (2015). Fouque and Ichiba (2013) considers an interbank model coupled through Feller processes and discover that multiple defaults can occur simultaneously in this model, while the handbook of Fouque and Langsam (2013) provide an overview of various theoretical and applied methods to model systemic risk. Bo and Capponi (2015) extends the framework by considering shocks to each bank's reserves, and Biagini et al. (2015) generalizes the framework to consider systemic risk measures.

In this paper, we extend the setup of Carmona et al. (2015) to banks that are willing to accept that their model for inter-bank lending may be wrong - in others that they account for model uncertainty (also called ambiguity aversion). Ambiguity aversion has been studied in many different context, and a limited list of contributions from a stochastic control perspective includes Uppal and Wang (2003), Maenhout (2004), Hansen et al. (2006), Hansen and Sargent (2007), Lim and Shanthikumar (2007), Jaimungal and Sigloch (2012), Skiadas (2013), and Cartea et al. (2014). Thus far, however, there has been only limited study of model uncertainty in the stochastic game context, and in particular in mean-field games (MFGs).

There are two main exceptions. The first is Huang and Huang (2017) where the authors consider a deterministic, but unknown, drift in a linear-quadratic stochastic game and solve the mean-field game problem by viewing the drift uncertainty as an adversarial player. Bauso et al. (2016) study a robust mean-field game problem where the drifts are 
indeed stochastic processes, but the Browniann drivers are all independent and there is no mean-field term in the players' drift dynamics - the mean-field appears only in the cost functional. They use the dynamic programming principle and apply their approach to the production of an exhaustible resource. Here, we study a problem that is more general in several ways. The most important is that we allow for a mean-field coupling in the dynamics, and not just the cost functional. Secondly, we allow for correlation in the Brownian drivers. Thirdly, we take the stochastic Pontryagin Maximum principle approach and provide explicit solutions to the finite game and mean-field game problems.

For stochastic mean-field games, uniqueness and existence results were first given by Lasry and Lions (2007a) and Huang et al. (2006), with more general results provided in Guéant et al. (2011), Cardaliaguet (2012) and Carmona and Delarue (2013). The results in Carmona and Delarue (2013) primarily rely on convexity of the cost functional, and much less is known for mean-field games with concave-convex cost functions.

For deterministic games with concave-convex cost functions, there is a direct relationship between the Hamiltonian and the cost function of each player, hence, the NashPontryagin principle, as introduced in Geering (2007), is easily derived. In the stochastic game context, the only work the authors are aware of is that of Bauso et al. (2016) where the authors assume the Brownian drivers are independent. The verification theorem we provide here encompasses a much larger class of models with ambiguity aversion and provides an explicit condition which the optimal controls and Hamiltonian function must satisfy.

The remainder of the paper is organized as follows. Section 3.2 introduces how ambiguity aversion is incorporated into the log-reserve dynamics. Section 4 solves and compares the closed-loop and open-loop controls. Section 5 derives the control in the mean-field game limit, and compares it with the finite player controls. Section 6 provides a verification theorem for the mean-field game problem. Section 7 illustrates the effect that ambiguity aversion has in the finite-player game, and how it impacts banks' default probabilities.

\subsection{The Model Setup}

To begin, as usual, let $\boldsymbol{W}=\left\{\boldsymbol{W}_{t}=\left(W_{t}^{0}, \ldots, W_{t}^{k}\right)^{\prime}\right\}_{t \geq 0}$ be an $N+1$-dimensional standard Brownian (uncorrelated components) defined on the filtered probability space $\left(\Omega, \mathcal{F},\left\{\mathcal{F}_{t}\right\}_{t \geq 0}, \mathbb{P}\right)$, where $\left\{\mathcal{F}_{t}\right\}_{t \geq 0}$ is the $\mathbb{P}$-augmentation of the natural filtration generated by $\boldsymbol{W}$.

Carmona et al. (2015) consider a framework with $N$ banks borrowing/lending from/to one another. The log-monetary reserves is denoted $\left\{X^{i}=\left(X_{t}^{i}\right)_{t \geq 0}: i=1, \ldots, N\right\}$. The 
authors assume each bank will initially borrow (respectively lend) from banks with higher (resp. lower) reserves than their own, at a rate that is proportional to the difference. Hence, the log-monetary reserves are assumed to satisfy the (coupled) SDEs

$$
d X_{t}^{i}=\left\{\frac{a}{N} \sum_{k=1}^{N}\left(X_{t}^{k}-X_{t}^{i}\right)+\alpha_{t}^{i}\right\} d t+\sigma\left(\rho d W_{t}^{0}+\sqrt{1-\rho^{2}} d W_{t}^{i}\right), \quad i=1, \ldots, N .
$$

Here, $\alpha^{i}=\left(\alpha_{t}^{i}\right)_{t \geq 0}$ is an $\mathcal{F}$-adapted process and represents banks $i$ 's rate of borrowing/lending to the central bank. The rate $\alpha^{i}$ is what bank $i$ can control and we will denote the collection of controls by $\boldsymbol{\alpha}=\left(\alpha_{t}^{1}, \ldots, \alpha_{t}^{N}\right)_{t \geq 0}$. The parameter $\sigma$ represents the volatility of the bank's log-reserve, and can be viewed as the withdrawal/deposits made to/from the bank every day by, e.g., retail investors.

Each bank's objective is to minimize their own cost functional

$$
J^{i}(\boldsymbol{\alpha})=\mathbb{E}\left[\int_{0}^{T} f^{i}\left(\boldsymbol{X}_{t}, \alpha_{t}^{i}\right) d t+g^{i}\left(\boldsymbol{X}_{T}\right)\right]
$$

with running cost

$$
f^{i}\left(\boldsymbol{x}, \alpha^{i}\right)=\frac{1}{2}\left(\alpha^{i}\right)^{2}-q \alpha^{i}\left(\bar{x}-x^{i}\right)+\frac{\epsilon}{2}\left(\bar{x}-x^{i}\right)^{2},
$$

and terminal cost

$$
g^{i}(\boldsymbol{x})=\frac{c}{2}\left(\bar{x}-x^{i}\right)^{2},
$$

and where $\bar{x} \triangleq \frac{1}{N} \sum_{j=1}^{N} x^{j}$. When $\epsilon=c=0, q$ can be interpreted as the cost to borrow/lend from the central bank, with large q being akin to low fees. In general, banks want to follow the average log-reserves of other banks, with the parameter $q$ representing the cost of borrowing and lending from the central bank (versus borrowing/lending from other banks). The performance criteria is composed of the cost of borrowing/lending, the penalty for deviating from the average log-reserves, and the relative benefit of borrowing/lending to the central bank versus borrowing/lending directly from other banks. Mathematically, we require the condition $q^{2}<\epsilon$ to be satisfied in order for the problem to be well-posed.

Carmona et al. (2015) derive the optimal control and established that a Nash equilibrium is achieved in the finite player case. They also demonstrate, in the limit as $N \rightarrow \infty$, the optimal control converges to the solution for the control in the mean-field game. Moreover, they demonstrate that within the Nash equlibria, each bank has a lower probability of default than what they would have if they individually optimized their cost 
functional and ignored the interactions.

Next, we will generalize the setup to account for ambiguity aversion (model uncertainty). Each player's ambiguity aversion is measured by the strength of an ambiguity aversion matrix $\boldsymbol{\Phi}_{i}$. Being ambiguity averse, implies that each bank will consider alternate models to the base case above, and distorts the dynamics in (3.1). The ambiguity aversion matrix allows each bank to penalize candidate models idiosyncratically with respect to the other banks. This notion is formalized in the reserve dynamics and performance criteria below.

\subsubsection{Reserve Dynamics}

When players are ambiguity averse, each player has their own perspective of the dynamics of the system. From the perspective of the $i^{\text {th }}$ ambiguity averse player, the dynamics of the log-reserves for the $k^{\text {th }}$ player satisfies the SDE

$$
d X_{t}^{i, k}=\left\{\frac{a}{N} \sum_{j=1}^{N}\left(X_{t}^{i, j}-X_{t}^{i, k}\right)+\alpha_{t}^{k}+\eta_{t}^{i, k}\right\} d t+\sigma\left(\rho d W_{t}^{0}+\sqrt{1-\rho^{2}} d W_{t}^{k}\right) .
$$

Here, $\eta^{i, k}=\left(\eta_{t}^{i, k}\right)_{t \geq 0}(\mathcal{F}$-adapted) is the ambiguous (stochastic) drift that player $i$ places in the dynamics of player $k$.

\subsubsection{Performance Criteria}

Each bank wishes to minimize her cost function (3.2), where the running cost for the $i^{\text {th }}$ bank is

$$
f^{i}\left(t, \boldsymbol{x}, \alpha^{i}, \boldsymbol{\eta}\right) \triangleq\left[\frac{1}{2}\left(\alpha^{i}\right)^{2}-q \alpha^{i}\left(\bar{x}-x^{i}\right)+\frac{\epsilon}{2}\left(\bar{x}-x^{i}\right)^{2}-\frac{1}{2} \eta^{T} \boldsymbol{\Phi}_{i}^{-1} \eta\right],
$$

and the terminal cost is

$$
g^{i}(\boldsymbol{x})=\frac{c}{2}\left(\bar{x}-x^{i}\right)^{2} .
$$

Here, $\boldsymbol{\Phi}_{i}$ is the ambiguity matrix of the $i^{\text {th }}$ agent which is assumed to be positive semidefinite, and $\eta^{i}=\left[\eta^{i, 1}, \ldots, \eta^{i, N}\right]$ is the vector of drift changes under a given measure change. This ambiguity penalty term can be viewed as stemming from the relative entropy between the reference model (with $\eta_{t}^{i, k}=0$ ) and the candidate model (with $\left.\eta_{t}^{i, k} \neq 0\right)$.

The $i^{t h}$ player aims to minimize her cost under all equivalent measures, and hence 
her cost function under the optimal strategy will be

$$
J^{i}(\boldsymbol{\alpha}, \boldsymbol{\eta})=\inf _{\alpha^{i} \in \mathcal{A}} \sup _{\eta^{i} \in \mathcal{B}} \mathbb{E}\left[\int_{0}^{T} f^{i}\left(\boldsymbol{X}_{t}, \alpha_{t}^{i}, \eta_{t}^{i}\right) d t+g^{i}\left(\boldsymbol{X}_{T}\right)\right]
$$

where the set of admissible controls $\mathcal{A}, \mathcal{B}$ are

$$
\mathcal{A}=\left\{\alpha_{t}^{i} \mid \mathbb{E}\left[\int_{0}^{T}\left(\alpha_{t}^{i}\right)^{2} d t\right]<\infty\right\} \quad \mathcal{B}=\left\{\eta_{t}^{i} \mid \mathbb{E}\left[\int_{0}^{T}\left\|\eta_{t}^{i}\right\|^{2} d t\right]<\infty\right\} \quad \forall i, k \in 1, \ldots, N
$$

The approach we take to solving this problem is a variation of the stochastic Pontryagin minimum principle.

\subsection{N Player Game}

For a finite $N$-player game, we define the drift and volatility process of the ambiguity averse players, from the perspective of the $i^{t h}$ player, to be where $b(t, \boldsymbol{x}, \boldsymbol{\alpha}, \boldsymbol{\eta}): \mathbb{R} \times \mathbb{R}^{n} \times$ $\mathbb{R}^{n} \times \mathbb{R}^{n} \mapsto \mathbb{R}^{n}$ and $\boldsymbol{\sigma}(t, \boldsymbol{x}): \mathbb{R} \times \mathbb{R}^{n} \mapsto \mathbb{R}^{n^{2}}$ where the players' dynamics follow

$$
d \boldsymbol{X}_{t}^{i}=\boldsymbol{b}^{i}\left(t, \boldsymbol{X}_{t}^{i}, \boldsymbol{\alpha}, \boldsymbol{\eta}\right) d t+\boldsymbol{\sigma}^{i}\left(t, \boldsymbol{X}_{t}\right)\left(\rho \mathbb{1} d W_{t}^{0}+\sqrt{1-\rho^{2}} d \boldsymbol{W}_{t}\right),
$$

In the current setting, the drift and volatility terms have the following form

$$
\begin{aligned}
\boldsymbol{b}^{i}\left(t, \boldsymbol{X}_{t}^{i}, \boldsymbol{\alpha}, \boldsymbol{\eta}\right) & =\left\{\frac{a}{N} \sum_{j=1}^{N}\left(\mathbb{1} X_{t}^{i, j}-\boldsymbol{X}_{t}^{i}\right)+\boldsymbol{\alpha}_{t}^{k}+\boldsymbol{\eta}_{t}^{i}\right\} \\
\boldsymbol{\sigma}^{i}\left(t, \boldsymbol{X}_{t}\right) & =\sigma \mathbb{1}
\end{aligned}
$$

and the Hamiltonian for the $i^{\text {th }}$ player $\mathcal{H}^{i}(t, \boldsymbol{x}, \boldsymbol{\alpha}, \boldsymbol{y}, \boldsymbol{\eta}): \mathbb{R} \times \mathbb{R}^{n} \times \mathbb{R}^{n} \times \mathbb{R}^{n} \mapsto \mathbb{R}^{n}$ is defined as

$$
\mathcal{H}^{i}(t, \boldsymbol{x}, \boldsymbol{\alpha}, \boldsymbol{y}, \boldsymbol{\eta}) \triangleq \boldsymbol{b}^{i}(t, \boldsymbol{x}, \boldsymbol{\alpha}, \boldsymbol{\eta}) \cdot \boldsymbol{y}+\operatorname{Tr}\left[\boldsymbol{\sigma}^{i}(t, \boldsymbol{x}) \boldsymbol{z}\right]+f^{i}(t, \boldsymbol{x}, \boldsymbol{\alpha}, \boldsymbol{\eta})
$$

We define the optimal controls

$$
\begin{array}{cc}
\widehat{\boldsymbol{\eta}}^{i}(t, \boldsymbol{x}, \boldsymbol{\alpha}, \boldsymbol{y})=\underset{\boldsymbol{\eta} \in \mathcal{A}}{\arg \sup } \mathcal{H}^{i}\left(t, \boldsymbol{x}, \boldsymbol{\alpha}, \boldsymbol{y}, \boldsymbol{\eta}^{i}\right) . & i=1, \ldots, N \\
\widehat{\alpha}^{i}(t, \boldsymbol{x}, \boldsymbol{y})=\underset{\alpha^{i} \in \mathcal{A}}{\arg \inf } \mathcal{H}^{i}\left(t, \boldsymbol{x}, \boldsymbol{\alpha}, \boldsymbol{y}, \widehat{\boldsymbol{\eta}}^{i}\right) . & i=1, \ldots, N
\end{array}
$$


and will show in Appendix 3.C that these controls correspond to the optimal controls to the original optimization problem.

The arguments $\boldsymbol{y}$ and $\boldsymbol{z}$ correspond to the state of the adjoint (or co-state), of the $i^{\text {th }}$ agent, processes: $\boldsymbol{Y}^{i}=\left(\boldsymbol{Y}_{t}^{i}\right)_{t \geq 0}$ and $\boldsymbol{Z}^{i}=\left(\boldsymbol{Z}_{t}^{i}\right)_{t \geq 0}$ which satisfy the backwards stochastic differential equation (BSDE)

$$
d \boldsymbol{Y}_{t}^{i}=-\nabla_{\boldsymbol{x}} \mathcal{H}^{i}\left(t, \boldsymbol{X}_{t}^{i}, \widehat{\boldsymbol{\alpha}}_{t}, \boldsymbol{Y}_{t}^{i}, \widehat{\boldsymbol{\eta}}_{t}^{i}\right) d t+\boldsymbol{Z}_{t}^{i} d \boldsymbol{W}_{t},
$$

for some $\mathcal{F}$-adapted processes $\left\{Z_{t}^{i, j, k}\right\}_{k}$.

In the interest of notational simplicity, we sometimes omit $\boldsymbol{z}$ in the Hamiltonian since, since the volatility is uncontrolled, and hence does not depend on $\boldsymbol{\alpha}$. However, the process $\boldsymbol{Z}_{t}$ will be reintroduced when proving the verification theorem.

\subsubsection{Ambiguity Averse Agents}

We wish to find a Nash equilibrium for the problem when agents are ambiguity averse. To achieve this, we introduce a so-called un-optimized Hamiltonian and apply the Pontryagin's Minimum Principle. To this end, we can write the un-optimized Hamiltonian explicitly as

$$
\begin{aligned}
\mathcal{H}^{i}\left(t, \boldsymbol{x}, \boldsymbol{\alpha}, \boldsymbol{y}^{i}, \boldsymbol{\eta}^{i}\right) \triangleq & {\left[a(\bar{x} \mathbb{1}-\boldsymbol{x})+(\boldsymbol{\alpha})^{\top}+\left(\boldsymbol{\eta}^{i}\right)^{\top}\right] \boldsymbol{y}^{i} } \\
& +\frac{1}{2}\left(\alpha^{i}\right)^{2}-q \alpha^{i}\left(\overline{\boldsymbol{x}}-x^{i}\right)+\frac{\epsilon}{2}\left(\overline{\boldsymbol{x}}-x^{i}\right)^{2}-\frac{1}{2}\left(\boldsymbol{\eta}^{i}\right)^{\top} \boldsymbol{\Phi}_{i}^{-1} \boldsymbol{\eta}^{i} .
\end{aligned}
$$

Since each player is ambiguity averse, they aim to find

$$
\widehat{\alpha}^{i}\left(t, \boldsymbol{x}, \boldsymbol{y}^{i}\right)=\underset{\alpha^{i}}{\arg \min } \max _{\boldsymbol{\eta}^{i}} \mathcal{H}^{i}\left(t, \boldsymbol{x}, \boldsymbol{\alpha}, \boldsymbol{y}^{i}, \boldsymbol{\eta}^{i}\right)=-y^{i, i}+q\left(\overline{\boldsymbol{x}}-x^{i}\right), \quad i=1, \ldots, N
$$

From (3.17), it is clear that

$$
\widehat{\boldsymbol{\eta}}^{i}\left(t, \boldsymbol{x}, \boldsymbol{\alpha}, \boldsymbol{y}^{i}\right) \triangleq \underset{\boldsymbol{\eta}^{i}}{\arg \max } \mathcal{H}^{i}\left(t, \boldsymbol{x}, \boldsymbol{\alpha}, \boldsymbol{y}^{i}, \boldsymbol{\eta}^{i}\right)=\boldsymbol{\Phi}_{i} \boldsymbol{y}^{i} . \quad i=1, \ldots, N
$$

\subsubsection{Closed-Loop Game}

In a closed loop game, each player has full information about the other players' strategies, resulting in a feedback control. In particular, each player knows the relevant cost functions, and as a result, the influence of their strategy on the optimal strategies of the other players'. In contrast, an open loop game (which is derived in 3.3.4), players do not correct for any interaction between each others' optimal strategies when deciding on 
their own strategy.

Note that this optimal point for $\widehat{\boldsymbol{\eta}}^{i}$ is independent of $t, \boldsymbol{x}$ and $\boldsymbol{\alpha}$. Substituting $\widehat{\boldsymbol{\eta}}^{i}$ and $\widehat{\boldsymbol{\alpha}}$ into (3.17), we have

$$
\begin{aligned}
\widehat{\mathcal{H}}^{i}(t, \boldsymbol{x}, \boldsymbol{y}) \triangleq & \mathcal{H}^{i}\left(t, \boldsymbol{x}, \widehat{\boldsymbol{\alpha}}(t, \boldsymbol{x}, \boldsymbol{y}), \boldsymbol{y}^{i}, \widehat{\boldsymbol{\eta}}^{i}(t, \boldsymbol{x}, \boldsymbol{\alpha}, \boldsymbol{y})\right) \\
= & \sum_{k=1}^{N}\left[a\left(\bar{x}-x^{k}\right)-y^{k, k}+q\left(\bar{x}-x^{k}\right)\right] y^{i, k}+\frac{1}{2} \boldsymbol{y}^{i \top} \boldsymbol{\Phi}_{i} \boldsymbol{y}^{i} \\
& +\frac{1}{2}\left(\alpha^{i}\right)^{2}-q \alpha^{i}\left(\bar{x}-x^{i}\right)+\frac{\epsilon}{2}\left(\bar{x}-x^{i}\right)^{2} \\
= & \sum_{k \neq i}\left[(a+q)\left(\bar{x}-x^{k}\right)-y^{k, k}\right] y^{i, k} \\
& +\left[(a+q)\left(\bar{x}-x^{i}\right)-\frac{1}{2} y^{i, i}\right] y^{i, i}+\frac{1}{2} \boldsymbol{y}^{i \top} \boldsymbol{\Phi}_{i} \boldsymbol{y}^{i}+\frac{\epsilon-q^{2}}{2}\left(\bar{x}-x^{i}\right)^{2}
\end{aligned}
$$

Given the dependency of the optimal controls on the adjoint process $\boldsymbol{Y}_{t}^{i}$, we can solve for the stochastic controls $\alpha_{t}^{i}, \eta_{t}^{i, k}:(t, x, y) \rightarrow \mathbb{R}$ explicitly as functions of $(t, x)$ by solving the system of forward-backwards stochastic differential equations given below. This allows us to find an FBSDE value function $u^{i}\left(t, \boldsymbol{X}_{t}\right)$ such that $u^{i}\left(t, \boldsymbol{X}_{t}\right)=\boldsymbol{Y}_{t}^{i}$ almost surely. (see Carmona and Delarue (2013) for details regarding the FBSDE value function. Going forward, we will use $Y_{t}^{i, k}$ to mean $u^{i, k}\left(t, \boldsymbol{X}_{t}\right)$, in the sense that it is a function of time and the state of the process at that time $\boldsymbol{X}_{t}$ )

$$
\begin{aligned}
d \boldsymbol{X}_{t}^{i} & =\nabla_{\boldsymbol{y}} \mathcal{H}^{i}\left(t, \boldsymbol{x}, \widehat{\boldsymbol{\alpha}}(t, \boldsymbol{x}, \boldsymbol{y}), \boldsymbol{y}, \widehat{\boldsymbol{\eta}}^{i}(t, \boldsymbol{x}, \boldsymbol{\alpha}, \boldsymbol{y})\right) d t+\sigma\left(\rho \mathbb{1} d W_{t}^{0}+\sqrt{1-\rho^{2}} d \boldsymbol{W}_{t}\right) \\
d \boldsymbol{Y}_{t}^{i} & =-\nabla_{\boldsymbol{x}} \mathcal{H}^{i}\left(t, \boldsymbol{x}, \widehat{\boldsymbol{\alpha}}(t, \boldsymbol{x}, \boldsymbol{y}), \boldsymbol{y}, \widehat{\boldsymbol{\eta}}^{i}(t, \boldsymbol{x}, \boldsymbol{\alpha}, \boldsymbol{y})\right) d t+\boldsymbol{Z}_{t}^{i} d \boldsymbol{W}_{t}
\end{aligned}
$$

For details regarding the link between FBSDEs and Hamiltonians, we refer the reader to Yong and Zhou (1999).

In the remainder of this section, we will assume the banks are exchangeable with respect to their ambiguity aversion levels, so that

$$
\mathbf{\Phi}_{i}=\mathbf{\Phi} \quad i=1, \ldots, N
$$

and we make the following ansatz for the collection of dual processes $\left\{Y^{i, k}\right\}_{k=1}^{N}$ based on the linear-quadratic form of the problem

$$
u^{i, k}\left(t, \boldsymbol{X}_{t}^{i}\right)=Y_{t}^{i, k}=\psi(t)\left(\frac{1}{N}-\delta_{i, k}\right)\left(\overline{\boldsymbol{X}}_{t}^{i}-X_{t}^{i}\right)
$$

where $\psi(t)$ is a deterministic function of time with the terminal condition $\psi(T)=c$. 


\subsubsection{FBSDE for closed-loop equilibria}

By the result in Carmona et al. (2015), the solution to this optimization problem satisfies the following forward-backwards stochastic differential equation

$$
\begin{aligned}
d \boldsymbol{X}_{t}^{i} & =\nabla_{\boldsymbol{y}} \mathcal{H}^{i}\left(t, \boldsymbol{X}_{t}^{i}, \widehat{\boldsymbol{\alpha}}\left(t, \boldsymbol{X}_{t}, \boldsymbol{Y}_{t}\right), \boldsymbol{Y}_{t}, \widehat{\boldsymbol{\eta}}^{i}\left(t, \boldsymbol{X}_{t}^{i}, \widehat{\boldsymbol{\alpha}}, \boldsymbol{Y}_{t}\right)\right) d t+\sigma\left(\rho \mathbb{1} d W_{t}^{0}+\sqrt{1-\rho^{2}} d \boldsymbol{W}_{t}\right) \\
d \boldsymbol{Y}_{t}^{i} & =-\nabla_{\boldsymbol{x}} \mathcal{H}^{i}\left(t, \boldsymbol{X}_{t}^{i}, \widehat{\boldsymbol{\alpha}}\left(t, \boldsymbol{X}_{t}, \boldsymbol{Y}_{t}\right), \boldsymbol{Y}_{t}, \widehat{\boldsymbol{\eta}}^{i}\left(t, \boldsymbol{X}_{t}^{i}, \widehat{\boldsymbol{\alpha}}, \boldsymbol{Y}_{t}\right)\right) d t+\boldsymbol{Z}_{t}^{i} d \boldsymbol{W}_{t}
\end{aligned}
$$

Theorem 3.3.1. If the processes $X_{t}^{i}$ are exchangeable, then the optimal control of the $i^{\text {th }}$ player is of the form

$$
\widehat{\alpha}_{t}^{i}\left(t, \boldsymbol{X}_{t}^{i}\right)=\left(q+\psi(t)\left(1-\frac{1}{N}\right)\right)\left(\overline{\boldsymbol{X}}_{t}^{i}-X_{t}^{i}\right)
$$

where $\psi_{t}$ satisfies the $O D E$

$$
\dot{\psi}_{t}=\left\{2(a+q)+\left(1-\frac{1}{N^{2}}\right) \psi_{t}\right\} \psi_{t}+\left\{\sum_{k=1}^{N}\left(\frac{1}{N}-\delta_{i, k}\right) \Phi_{i, k}\right\} \psi_{t}^{2}+\left(q^{2}-\epsilon\right), \quad \psi_{T}=c
$$

and where the ambiguity matrix is represented as $\Phi=\left\{\Phi_{i, j}\right\}_{i, j=1}^{N}$.

Proof. See Appendix 3.A

We note here that with $\Phi=[0]$, we recover the result in Carmona et al. (2015)

\subsubsection{Open-Loop Game}

In game theory, strategies for open-loop and closed-loop games generally do not coincide. When studying mean-field games, the question is whether the open and closed loop strategies coincide as $N \rightarrow \infty$. In particular, Carmona and Zhu (2016) show that when a major player is present in the game, there is a gap between these controls. Therefore, we solve the open loop game to verify whether this gap exists under ambiguity aversion as well.

Performing the same computations, but without considering $\alpha^{k}(t, x)$ as explicit functions of the state variables for $k \neq i$, we obtain a similar FBSDE

$$
\begin{aligned}
& d X_{t}^{i, i}=\left(\overline{\boldsymbol{X}}_{t}^{i}-X_{t}^{i}\right)(a+q)-Y_{t}^{i, i}+\Phi_{i, j} \cdot \boldsymbol{Y}_{t}^{i} d t+\sigma\left(\rho \mathbb{1} d W_{t}^{0}+\sqrt{1-\rho^{2}} d W_{t}^{j}\right) \\
& d Y_{t}^{i, j}=\left(\delta_{i, j}-\frac{1}{N}\right)\left(\overline{\boldsymbol{X}}_{t}^{i}-X_{t}^{i}\right)\left[\left(a+\left(1-\frac{1}{N}\right) q\right) \psi_{t}-\left(\epsilon-q^{2}\right)\right] d t+\boldsymbol{Z}_{t}^{i, j} d \boldsymbol{W}_{t}
\end{aligned}
$$


and as a result derive a similar ODE for the open-loop control of the form

$$
\hat{\alpha}_{t}^{i}\left(t, \boldsymbol{X}_{t}^{i}\right)=\left(q+\psi(t)\left(1-\frac{1}{N}\right)\right)\left(\overline{\boldsymbol{X}}_{t}-X_{t}^{i}\right)
$$

where $\psi_{t}$ is the solution to the following ODE

$$
\dot{\psi}_{t}=\left(2(a+q)+\left(1-\frac{1}{N}\right) \psi_{t}\right) \psi_{t}+\left(\sum_{k=1}^{N}\left(\frac{1}{N}-\delta_{i, k}\right) \Phi_{i, k}\right) \psi_{t}^{2}+\left(q^{2}-\epsilon\right), \quad \psi_{T}=c
$$

Comparing the expressions in (3.23) and (3.26), we can see that in the limit as $N \rightarrow \infty$, the open-loop and closed-loop control converge to the same function. This is contrary to what was found in Carmona and Zhu (2016) when the open-loop and closed-loop strategies were compared with a major agent present. Although the ambiguity averse dynamics act as a major player against each bank, the gap between the open-loop and closed-loop in this case would only have occurred if each player had ignored the effect that ambiguity aversion $\left(\eta^{i}\right)$ had on their cost function and dynamics, and in that case, the open-loop problem would have reduced to the original one in Carmona et al. (2015).

\subsection{Mean-field Game}

The previous section provided the analysis for the optimal control in the finite player game. This section deals with the mean-field version of the game. The mean-field formulation provides an approximate control for the finite player game, while allowing the exchangeability property to be relaxed to a certain extent.

\subsubsection{The Mean-Field}

As usual, in the mean-field game the average reserve $\frac{1}{N} \sum_{i=1}^{N} X_{t}^{i}$ is replaced by a meanfield term $m\left(t, \mu_{t}\right)$, where $\mu_{t}$ is the mean-field measure flow of log-reserves at time t. One then solves the auxiliary control problem and searches for a fixed point to consistently determine the mean-field.

\subsubsection{Setup}

In the mean-field game setting, we model the $i^{t h}$ ambiguity averse agent by assuming their log-reserve $X^{i}$ satisfies the SDE

$$
d X_{t}^{i}=\left\{a\left(m\left(t, \mu_{t}\right)-X_{t}^{i}\right)+\alpha_{t}^{i}+\eta_{t}^{i}\right\} d t+\sigma\left(\rho d W_{t}^{0}+\sqrt{1-\rho^{2}} d W_{t}^{i}\right) .
$$


The running cost function for the $i^{\text {th }}$ agent is

$$
f^{i}(x, \alpha, \eta ; \mu)=\left[\frac{1}{2}(\alpha)^{2}-q \alpha\left(m\left(t, \mu_{t}\right)-x\right)+\frac{\epsilon}{2}\left(m\left(t, \mu_{t}\right)-x\right)^{2}-\frac{1}{2 \phi_{i}}\left(\eta^{i}\right)^{2}\right],
$$

and the terminal cost is

$$
g^{i}(x ; \mu)=\frac{c}{2}\left(m\left(T, \mu_{T}\right)-x\right)^{2} .
$$

In contrast to the $\mathrm{N}$-player setting, $\phi_{i}$ in the mean-field game is a positive scalar representing the $i^{\text {th }}$ agent's ambiguity aversion. For an ambiguity averse agent, the goal is to minimize the penalized expected cost under all misspecified models.

$$
J^{i}\left(\alpha^{i}, \eta^{i}\right)=\min _{\alpha^{i} \in \mathcal{A}} \max _{\eta^{i} \in \mathcal{B}} \mathbb{E}\left[\int_{0}^{T} f^{i}\left(X_{t}^{i}, \alpha^{i}, \eta^{i} ; \mu_{t}\right) d t+g^{i}\left(X_{T}^{i} ; \mu_{T}\right)\right] .
$$

In the mean-field setting, we require an additional assumption on the initial states $X_{0}^{i}$ and the ambiguity parameters $\phi_{i}$

Assumption 3.4.1. Each agent's initial state $X_{0}^{i}$ is independent and identically distributed according to some random variable $\xi$. Furthermore, we assume that $\mathbb{E}\left[(\xi)^{2}\right]<\infty$ and $\sup _{i}\left|\phi_{i}\right|<C$ for some $C \in \mathbb{R}^{+}$.

\section{Outline of Approach}

To solve the MFG problem, we set $m=\left(m_{t}\right)_{t \geq 0}$ where

$$
m_{t}=m\left(t, \mu_{t}\right)=\int x d \mu_{t}(x)
$$

for a given frozen measure flow $\mu=\left(\mu_{t}\right)_{t \geq 0}$. Then we solve the one-dimensional control problem for an arbitrary agent to find the optimal control $\alpha_{t}$ under the frozen measure flow. Finally, the consistency equation $m_{t}=\mathbb{E}\left[X_{t} \mid\left(W_{s}^{0}\right)_{s \leq t}\right]$, if a fixed-point exists, leads to the optimal control for player $i$ in the mean-field equilibrium.

\section{Derivation}

We first assume a frozen measure flow $\mu_{t}$, so that the mean-field $m_{t}$ is a function $m\left(t, \mu_{t}\right)$. The unoptimized Hamiltonian in this case is

$$
\mathcal{H}^{i}\left(t, x^{i}, \alpha_{t}, y, \eta_{t}^{i} ; m_{t}\right)=\left[a\left(m_{t}-x^{i}\right)+\alpha_{t}+\eta_{t}^{i}\right] y+\frac{1}{2}\left(\alpha_{t}\right)^{2}-q \alpha_{t}\left(m_{t}-x^{i}\right)+\frac{\epsilon}{2}\left(m_{t}-x^{i}\right)^{2}-\frac{\left(\eta_{t}^{i}\right)^{2}}{2 \phi_{i}}
$$


so, similar to the finite closed-loop game, we find the optimal controls by optimizing with respect to the Hamiltonian

$$
\widehat{\mathcal{H}}^{i}\left(x, y ; m_{t}\right) \triangleq \inf _{\alpha_{t}^{i} \in \mathcal{A}} \sup _{\eta_{t}^{i} \in \mathcal{B}} \mathcal{H}^{i}\left(t, x, y, \alpha_{t}^{i}, \eta_{t}^{i} ; m_{t}\right)=\mathcal{H}^{i}\left(t, x, y, \widehat{\alpha}_{t}^{i}, \widehat{\eta}_{t}^{i} ; m_{t}\right)
$$

where the sets $\mathcal{A}, \mathcal{B}$ are defined in (3.9).

The solution to this optimization problem yields the optimal controls in feedback form

$$
\begin{aligned}
\hat{\eta}^{i}\left(t, x, \alpha^{i}, y ; m_{t}\right) & =\phi_{i} y \\
\hat{\alpha}^{i}\left(t, x, y ; m_{t}\right) & =-y+q\left(m_{t}-x^{i}\right)
\end{aligned}
$$

Therefore, the Hamiltonian can be written as

$$
\widehat{\mathcal{H}}^{i}\left(t, x, y ; m_{t}\right)=\left[(a+q)\left(m_{t}-x\right)-\frac{1}{2}\left[1-\phi_{i}\right] y\right] y+\frac{\epsilon-q^{2}}{2}\left(m_{t}-x\right)^{2}
$$

By applying the same techniques as in the N-player game, we can characterize the dynamics for the log-reserve $X_{t}^{i}$ as well as the adjoint process $Y_{t}^{i}$ through the following forward-backward stochastic differential equation

$$
\begin{aligned}
& d X_{t}^{i}=\left[(a+q)\left(m_{t}-X_{t}^{i}\right)-\left[1-\phi_{i}\right] Y_{t}^{i}\right] d t+\sigma\left(\rho d W_{t}^{0}+\sqrt{1-\rho^{2}} d W_{t}\right), \quad X_{0}=\xi \\
& d Y_{t}^{i}=\left[(a+q) Y_{t}^{i}+\left(\epsilon-q^{2}\right)\left(m_{t}-X_{t}^{i}\right)\right]+\sum_{k=0}^{N} Z_{t}^{i, k} d W_{t}^{k}, \quad Y_{T}=c\left(X_{t}^{i}-m_{t}\right)
\end{aligned}
$$

Theorem 3.4.1. If $\sup _{k} \phi_{k}<1$ then the optimal control for the $i^{\text {th }}$ player is of the form

$$
\widehat{\alpha}_{t}^{i}\left(t, x, y ; m_{t}\right)=(q+\psi(t))\left(m_{t}-x^{i}\right)
$$

where $\psi_{t}$ admits the following closed-form representation

$$
\psi(t)=\frac{-\left(\epsilon-q^{2}\right)\left(e^{\left(\delta^{+}-\delta^{-}\right)(T-t)}-1\right)-c\left(\delta^{+} e^{\left(\delta^{+}-\delta^{-}\right)(T-t)}-\delta^{-}\right)}{\left(\delta^{-} e^{\left(\delta^{+}-\delta^{-}\right)(T-t)}-\delta^{+}\right)-c\left(1-\phi_{i}\right)\left(e^{\left(\delta^{+}-\delta^{-}\right)(T-t)}-1\right)}
$$

where

$$
\delta^{ \pm}=-(a+q) \pm \sqrt{R}
$$

and

$$
R=(a+q)^{2}+\left(\epsilon-q^{2}\right)\left(1-\phi_{i}\right)
$$

Proof. See Appendix 3.B 
If we compare to the form of the solution in Carmona et al. (2015), we see that they obtain the same form of the solution for a finite player game without ambiguity aversion by having $\left(1-\frac{1}{N}\right)$ in the denominator instead of $\left(1-\phi_{i}\right)$. In addition, this solution is consistent with the limit of the open-loop and closed-loop solution of the finite player game when we take

$$
\phi_{i}=\Phi_{i, i}-\lim _{N \rightarrow \infty} \frac{1}{N} \sum_{k=1}^{N} \Phi_{i, k}
$$

which is positive, since $\Phi_{i}$ was taken to be a positive definite matrix. In financial terms, this result tells us that the uncertainty of a player's dynamics should be the difference between the uncertainty towards their own reserve dynamics and the average uncertainty towards everyone else's reserve dynamics. As the goal of ambiguity aversion is to create a robust control for each player, it would be natural for a player to be more ambiguity averse to the Brownian Motion driving their own reserves.

In addition, since we did specify any constraints on the ambiguity aversion parameters other than that they be positive (or the matrix be positive-definite), the mean-field game can accommodate different levels of ambiguity aversion as well as different levels of volatility, given that these values are independent of the stochastic log-reserves at time 0 for all $X_{0}^{i}, \quad i=1, \ldots, N$.

\subsection{Verification Theorem and $\epsilon$-Nash Equilibrium}

The game being considered in this paper can be broken into two parts. The first one is a zero-sum stochastic game between the drift $\eta^{i}$ of the misspecified Brownian motion and the player's control $\alpha^{i}$. The second is between the control of player i, $\alpha^{i}$, and the other players' dynamics and controls. Zero-sum stochastic games have been studied extensively, but to our knowledge, the method of applying a stochastic Nash-Pontryagin method has not been used. The deterministic case for a Nash-Pontryagin principle has been studied previously in Geering (2007), using variational methods. As our setting includes a meanfield component as well as a (correlated) Brownian motion, we feel it is appropriate to provide a proof of the verification theorem for a stochastic Nash-Pontryagin method using arguments relying on the convexity and concavity properties of the Hamiltonian.

Theorem 3.5.1. Verification Theorem

Assume that there exists a solution $\hat{Y}_{t}, \hat{Z}_{t}$ to the BSDE

$$
d \hat{Y}_{t}=-\partial_{x} \widehat{\mathcal{H}}\left(t, \hat{X}_{t}, \hat{\alpha}_{t}, \hat{Y}_{t}, \hat{Z}_{t}, \hat{\eta}_{t}\right) d t+\hat{Z}_{t} d W_{t}, \quad Y_{T}=g\left(X_{T}\right)
$$


such that

$$
\widehat{\mathcal{H}}\left(t, \hat{X}_{t}, \hat{\alpha}_{t}, \hat{Y}_{t}, \hat{Z}_{t}, \hat{\eta}_{t}\right)=\min _{\alpha \in A} \max _{\eta \in E} \mathcal{H}\left(t, \hat{X}_{t}, \alpha, \hat{Y}_{t}, \hat{Z}_{t}, \eta\right)
$$

and

$$
\mathcal{H}\left(t, x, \alpha_{t}, y, z, \hat{\eta}_{t}(x, y, z)\right)=\max _{\eta \in \mathcal{B}} \mathcal{H}(t, x, \alpha, y, z, \eta)
$$

is convex in $(\alpha, x)$ and

$$
\mathcal{H}\left(t, x, \hat{\alpha}_{t}(x, y, z), y, z, \eta_{t}\right)=\min _{\alpha \in A} \mathcal{H}(t, x, \alpha, y, z, \eta)
$$

is concave is $(y, \eta)$ and convex in $x$.

Given the mean-field-game measure flow $\mu_{t}$, the optimal strategy of the $i^{\text {th }}$ agent $\alpha^{i}(t)$ minimizes her cost function $J\left(\alpha_{i} ; \alpha_{-i}\right)$, so that for any strategy $\alpha_{i} \in \mathcal{A}$ and

$$
J\left(\alpha_{i}, \hat{\eta}^{i}\right) \geq J\left(\hat{\alpha}_{i}, \hat{\eta}^{i}\right), \quad \forall i
$$

and $\eta^{i}$ maximizes the cost function such that for any alternative strategy $\eta^{i} \in \mathcal{E}$, and for the optimal $\alpha^{i} \in \mathcal{A}$ we have

$$
J\left(\hat{\alpha}_{i}, \eta^{i}\right) \leq J\left(\hat{\alpha}_{i}, \hat{\eta}^{i}\right)
$$

and hence

$$
J(\hat{\alpha}, \hat{\eta})=\min _{\alpha \in A} \max _{\eta \in E} J(\alpha, \eta)=\max _{\eta \in E} \min _{\alpha \in A} J(\alpha, \eta)
$$

Proof. See Appendix 3.C

One note we will make about this result is that this verification theorem holds if we reach a Nash equilibrium. However, a more general form of the verification can be done for the upper and lower values of a game if the optimal feedback controls $\hat{\alpha}, \hat{\eta}$ depend on each other. The existence of a unique minimax value is true when the two controls are separable in the drift of the process and the cost function, and a similar result was established for the deterministic control case in Geering (2007).

In terms of the finite and mean-field games we established previously, the only nontrivial condition to verify is that $\mathcal{H}\left(t, X_{t}, \hat{\alpha}_{t}\left(t, X_{t}, Y_{t}\right), Y_{t}, Z_{t}, \eta_{t}\right)$ is concave in $\left(Y_{t}, \eta_{t}\right)$. This condition is satisfied if $\phi<1$, which is precisely the bound for the optimal control $\widehat{\alpha}$ to have a solution. Therefore, this condition is necessary and sufficient for an equilibrium to exist. 


\subsection{1 $\epsilon$-Nash Equilibrium}

As games do not have infinitely many players, we establish an $\epsilon$-Nash equilibrium for the players in the mean-field game, which shows that the mean-field optimal controls are approximations of the optimal control in the finite player game.

Theorem 3.5.2. Under (Assumption 3.4.1), given $N$ players in the game, and an arbitrary strategy $\beta$, the optimal strategy $\alpha$ satisfies the following inequality

$$
J^{N}(\beta) \geq J^{N}(\alpha)+\epsilon_{N}
$$

where $\epsilon_{N} \rightarrow 0$ as $N \rightarrow \infty$

Proof. In the following proof, we use $O_{p}(X)$ to denote stochastic boundedness of a random variable by $X . \bar{X}^{N}, J^{N}$ denote the average log-reserve and cost function in the finite player game, while $\bar{X}, \bar{J}$ denote the average log-reserve and cost function in the meanfield game.

First, we assert that

$$
\left|\bar{X}-\bar{X}^{N}\right|=O_{p}\left(\frac{1}{\sqrt{N}}\right)
$$

which comes from the fact that under $\mu$, the mean-field is simply $\sigma \rho W_{t}^{0}$, and so

$$
d\left(\bar{X}_{t}^{N}-\bar{X}_{t}\right)=\frac{1}{N} \sum_{i=1}^{N}\left(a+q+\psi_{i}\right)\left(\bar{X}_{t}^{N}-\bar{X}_{t}\right)+\frac{1}{N} \sqrt{1-\rho^{2}} \sigma \sum_{k=1}^{N} d W_{t}^{k}
$$

and so there is a standard solution to this stochastic differential equation of the form

$$
\bar{X}_{t}^{N}-\bar{X}_{t}=\left(\bar{X}_{0}^{N}-\bar{X}_{0}\right) e^{-(a+q) t-\bar{\psi}(t)}+\sqrt{\frac{\left(1-\rho^{2}\right) \sigma^{2}}{N}} \int_{0}^{t} e^{-\int_{s}^{t}(a+q+\bar{\psi}(u)) d u} d W_{s}
$$

As a result, the $i^{\text {th }}$ player's dynamics are

$$
d X_{t}^{i}=\left(\left(a+q+\psi_{i}\right)\left(\bar{X}_{t}-X_{t}^{i}\right)\right) d t+\sigma\left(\rho d W_{t}^{0}+\left(\sqrt{1-\rho^{2}}\right) d W_{t}^{i}\right)+\delta_{N}
$$

where $\delta_{N}=O_{p}\left(\frac{1}{\sqrt{N}}\right)$

By law of large numbers, we have that $X_{0}^{N}=\bar{X}_{0}+O_{p}\left(\frac{1}{N}\right)$, so that

$$
\left|\bar{X}-\bar{X}^{N}\right|=O_{p}\left(\frac{1}{\sqrt{N}}\right)
$$

and by continuity of the process $\bar{X}_{t}, X_{t}^{i}$ and compactness of the time interval $[0, T]$ gives 
us

$$
\left|J^{N}(\alpha)-\bar{J}(\alpha)\right|=O\left(\frac{1}{\sqrt{N}}\right)
$$

Now, assume that player $\mathrm{j}$ takes an arbitrary control $\beta_{t}$ instead of the optimal control $\alpha_{t}$. In this case, we can replicate the computations above to obtain that

$$
\bar{X}_{t}^{N}=\bar{X}_{t}+O_{p}\left(\frac{1}{\sqrt{N}}\right)
$$

and

$$
J^{N}(\beta)=\bar{J}(\beta)+O\left(\frac{1}{\sqrt{N}}\right)
$$

Therefore, by combining the two results this gives us the desired result

$$
\left|J^{N}(\beta)-\bar{J}(\alpha)\right|=O\left(\frac{1}{\sqrt{N}}\right)
$$

As a result, we have an $\epsilon$-Nash equilibrium.

\subsection{Default Probability}

\subsubsection{PDE Approach}

Although an analytic solution is not available for the default probability of a particular bank, we can still compute a semi-analytic respresentation for the probability of default in the case where agents have homogeneous ambiguity aversion parameters.

Theorem 3.6.1. Given $N$ players with dynamics $\left\{X_{t}^{i}\right\}_{i=1}^{N}$, default levels $D$ and $\phi^{(1)}, \phi^{(2)} \in$ $\mathbb{R}^{+}$, there exists an integer $N_{0}$ such that if $N>N_{0}$, and $X_{0}^{(1)}=X_{0}^{(2)}<\overline{\boldsymbol{X}}_{0}$ then

$$
\phi^{(1)}>\phi^{(2)} \Longrightarrow \mathbb{P}\left(\min _{t} X_{t}^{(1)}<D\right)<\mathbb{P}\left(\min _{t} X_{t}^{(2)}<D\right)
$$

where $X_{t}^{(1)}$ (resp. $\left.X_{t}^{(2)}\right)$ is the log-reserve of a player at time $t$ when all players have ambiguity aversion level $\phi^{(1)}$ (resp. $\left.\phi^{(2)}\right)$.

Proof. See Appendix 3.D

Corollary 3.6.1. In the mean-field game, if two players have different levels of ambiguity $\phi_{i}, \phi_{j}<1$, with initial log-reserves $X_{0}^{i}=X_{0}^{j}<\overline{\boldsymbol{X}}$ and default level $D$, then

$$
\phi_{i}>\phi_{j} \Longrightarrow \mathbb{P}\left(\min _{t} X_{t}^{i}<D\right)<\mathbb{P}\left(\min _{t} X_{t}^{j}<D\right)
$$




\subsubsection{Simulations}

We verify the theoretical derivations using simulated results. As shown in Figure 3.2, banks will have a stronger control when they are ambiguity averse.

Default probabilities can be simulated for various levels of default. These simulations support the observation that ambiguity aversion decreases the probability of default for an agent significantly.

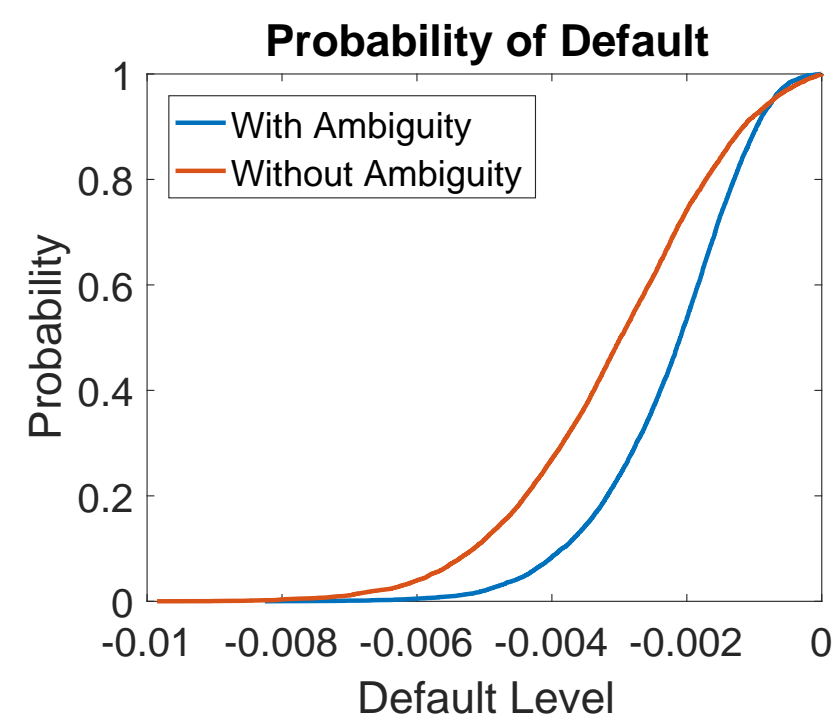

Figure 3.1: Simulated default probabilities for banks based on distance from default level

The simulations in Figure 3.1 included 10 banks, with the x-axis denoting the distance to default, and the y-axis denotes the probability that the bank will default before time $\mathrm{T}=1$. In general, banks will decrease their probability of default if they increase their level of ambiguity aversion. The anomaly observed around default level 0 is due to the approximate nature of the MFG control. In the MFG context, the probability of default will always decrease based on their level of ambiguity aversion due to Corollary 3.6.1.

In the following figures, the effect of ambiguity aversion on the strength of the control can be seen. Figure 3.2 plots the relative strength of the control based on the deviation from the mean $\frac{\alpha_{t}}{\left(m_{t}-x_{t}\right)}=q+\left(1-\frac{1}{N}\right) \psi(t)$. In comparing Figures 3.3 and 3.4 it should be noted that ambiguity aversion forces banks to maintain a reserve level which is much closer to the average log-reserve of all banks. 


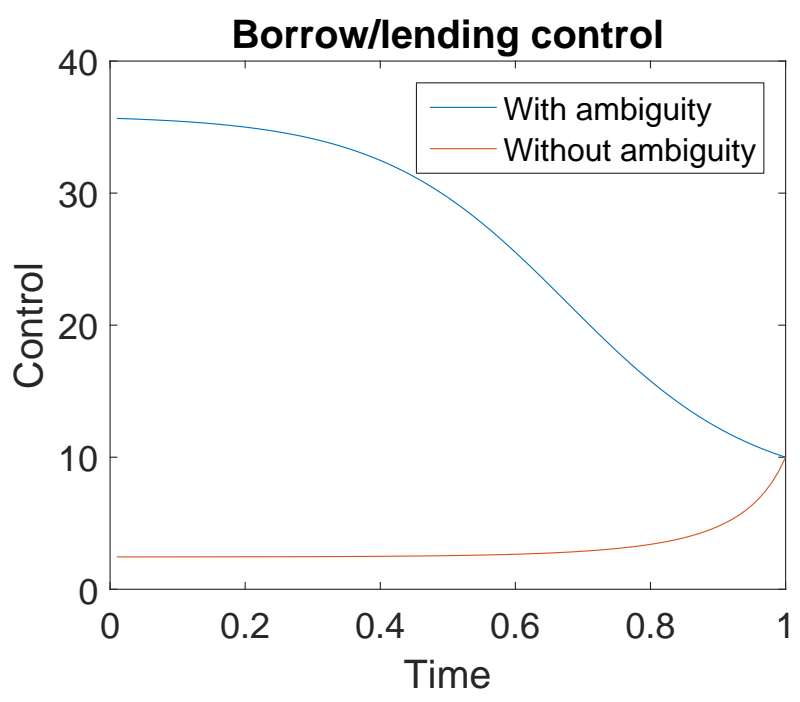

Figure 3.2: Optimal Control for banks $\alpha_{t}$

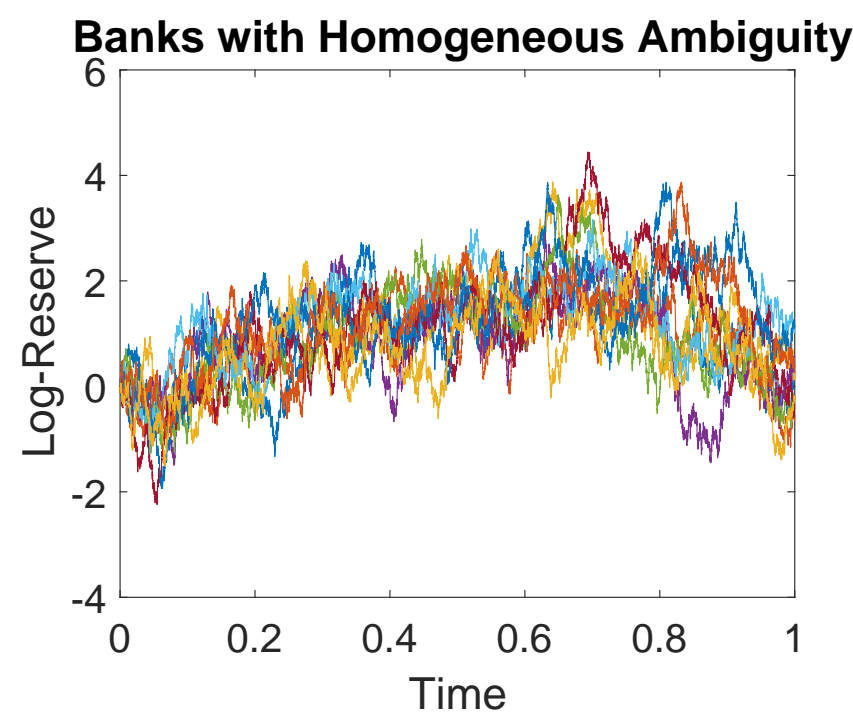

Figure 3.3: Sample paths with ambiguity parameter $\phi=1.5$

\subsubsection{Financial Implications}

The implications of these results are relevant for when a bank wishes to employ a strategy which is robust with respect to the actions of other banks. In this case, the introduction of ambiguity aversion will force the bank to stay closer to the average reserve amount to reduce their exposure. Although the bank will pay a higher cost to employ this strategy, they also reduce their probability of default as a result. In short

- The effect of ambiguity aversion decreases as the correlation with respect to com- 


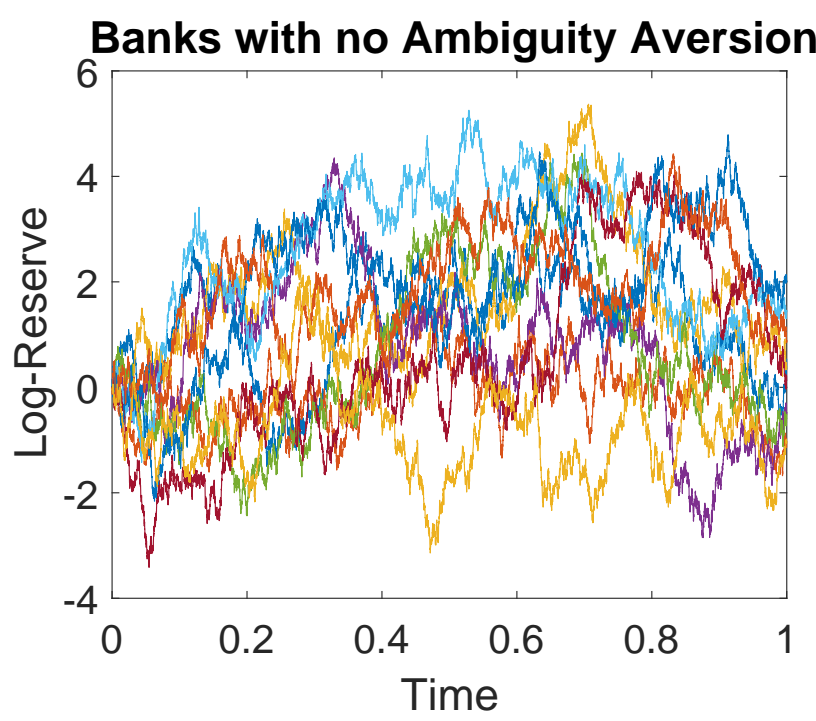

Figure 3.4: Sample paths with ambiguity parameter $\phi=0$

mon noise increases.

- Given a large number of players in the game, banks with a higher ambiguity aversion have a lower probability of default.

\subsection{Concluding Remarks}

This paper presents a framework that introduces model ambiguity into the log-reserve process for banks. The optimal control is derived, and the resulting dynamics are found to be less prone to default than in the model with no ambiguity aversion. The verification theorem derived can be employed in much more general models, including those with a stochastic volatility process.

Interesting questions for future research would be the effect of the central bank as a major player in the game, as well as the effect of ambiguity aversion when an explicit penalty for default is included in the performance criteria.

Other future work includes generalizing the concept of ambiguity aversion to the entire mean-field distribution with dynamics as in Carmona and Delarue (2013), as opposed to just the average of the mean-field which was considered here. 


\section{Appendix}

\section{A FBSDE for closed form solution}

Let

$$
\Phi=\left(\begin{array}{c}
\Phi_{1} \\
\vdots \\
\Phi_{N}
\end{array}\right)
$$

The partial derivatives which we must compute for the FBSDE are

$$
\begin{aligned}
\partial_{y^{i, j}} \mathcal{H}^{i} & =(a+q)\left(\overline{\boldsymbol{X}}_{t}^{i}-X_{t}^{i, i}\right)-Y^{j, j}+\Phi_{j} \cdot \boldsymbol{Y}^{i} \\
\partial_{x^{j}} \mathcal{H}^{i} & =-\left(\frac{1}{N}-\delta_{i, j}\right)\left(\overline{\boldsymbol{X}}_{t}^{i}-X_{t}^{i, i}\right)\left[\left(a+q+\frac{1}{N}\left(1-\frac{1}{N}\right) \psi_{t}\right) \psi_{t}+\left(q^{2}-\epsilon\right)\right]
\end{aligned}
$$

The dynamics of $X_{t}^{j}, j \neq i$ in the FBSDE match the ambiguity averse dynamics by definition. For notational simplicity, we will denote $\Phi=\left\{\Phi_{i, j}\right\}_{i, j=1, \ldots, N}$. The FBSDE implies that the player dynamics $d X_{t}^{i, k}$ can be written as

$$
d X_{t}^{i, k}=\left[(a+q)\left(\overline{\boldsymbol{X}}_{t}^{i}-X_{t}^{i, k}\right)-Y^{i, i}+\Phi_{k} \cdot \boldsymbol{Y}^{i}\right] d t+\sigma\left(\sqrt{1-\rho^{2}} d W_{t}^{i}+\rho d W_{t}^{0}\right)
$$

and through summation of each player's ambiguity averse dynamics we obtain the aggregate ambiguity-averse dynamics yielding

$$
d \overline{\boldsymbol{X}}_{t}^{i}=\left[\frac{\sum_{k=1}^{N}\left\langle\Phi_{k}, \boldsymbol{Y}^{i}\right\rangle}{N}\right] d t+\sigma\left(\rho d W_{t}^{0}+\sqrt{1-\rho^{2}} \frac{1}{N} \sum_{i=1}^{N} d W_{t}^{i}\right)
$$

and since the agents are exchangeable, we have that $\sum_{k=1}^{N} \Phi_{i, k}=\sum_{k=1}^{N} \Phi_{j, k}$ for all $1 \leq$ $i, j \leq N$ so that

$$
d \overline{\boldsymbol{X}}_{t}^{i}=\sigma\left(\rho d W_{t}^{0}+\sqrt{1-\rho^{2}} \frac{1}{N} \sum_{i=1}^{N} d W_{t}^{i}\right)
$$


Using the backward-equation form for $Y_{t}^{i, j}$, we obtain

$$
d Y_{t}^{i, j}=\left(\frac{1}{N}-\delta_{i, j}\right)\left(\overline{\boldsymbol{X}}_{t}^{i}-X_{t}^{i, i}\right)\left[\left(a+q+\frac{1}{N}\left(1-\frac{1}{N}\right) \psi_{t}\right) \psi_{t}+\left(q^{2}-\epsilon\right)\right]+\sum_{k=1}^{N} Z_{t}^{i, j, k} d W_{t}^{k}
$$

However, by differentiating the ansatz, we can see that

$$
\begin{array}{r}
d Y_{t}^{i, j}=\left(\frac{1}{N}-\delta_{i, j}\right)\left(\overline{\boldsymbol{X}}_{t}^{i}-X_{t}^{i, i}\right)\left[\dot{\psi}_{t}-(a+\right. \\
\left.\left.+q+\left(1-\frac{1}{N}\right) \psi_{t}+\left(\sum_{k=1}^{N}\left(\frac{1}{N}-\delta_{i, k}\right) \Phi_{i, k}\right) \psi_{t}\right) \psi_{t}\right] \\
\left.+\psi_{t} \sigma \sqrt{1-\rho^{2}}\left(\frac{1}{N}-\delta_{i, j}\right) \sum_{k=1}^{N}\left(\frac{1}{N}-\delta_{i, k} d W_{t}^{k}\right)\right)
\end{array}
$$

Comparing expressions we obtain

$$
Z_{t}^{i, j, k}=\psi_{t} \sigma \sqrt{1-\rho^{2}}\left(\frac{1}{N}-\delta_{i, j}\right)\left(\frac{1}{N}-\delta_{i, k}\right)
$$

and

$$
\dot{\psi}_{t}-\left(a+q+\left(1-\frac{1}{N}\right) \psi_{t}+\left(\sum_{k=1}^{N}\left(\frac{1}{N}-\delta_{i, k}\right) \Phi_{i, k}\right) \psi_{t}\right) \psi_{t}=\left(a+q+\frac{1}{N}\left(1-\frac{1}{N}\right) \psi_{t}\right) \psi_{t}+\left(q^{2}-\epsilon\right)
$$

so that the resulting ODE is

$$
\dot{\psi}_{t}=\left(2(a+q)+\left(1-\frac{1}{N^{2}}\right) \psi_{t}\right) \psi_{t}+\left(\sum_{k=1}^{N}\left(\frac{1}{N}-\delta_{i, k}\right) \Phi_{i, k}\right) \psi_{t}^{2}+\left(q^{2}-\epsilon\right)
$$

\section{B Mean-Field Game Optimal Control}

The mean processes for $X_{t}^{i}, Y_{t}^{i}$ can be written as

$$
m_{t}^{X^{i}}=\mathbb{E}\left[X_{t}^{i} \mid\left(W_{s}^{0}\right)_{s \leq t}\right] \quad m_{t}^{Y^{i}}=\mathbb{E}\left[Y_{t}^{i} \mid\left(W_{s}^{0}\right)_{s \leq t}\right]
$$

By taking a conditional expectation in the dynamics for $Y_{t}$, we obtain the following expression for the mean of $Y_{t}$

$$
m_{t}^{Y^{i}}=m_{t}^{Y}=-\int_{t}^{T} e^{(a+q)(s-t)} Z_{s}^{0} d W_{s}^{0}
$$


Similarly, by taking conditional expectation in the expression of $d X_{t}^{i}$, we obtain

$$
d m_{t}^{X^{i}}=-\left[1-\phi_{i}\right] m_{t}^{Y} d t+\rho \sigma d W_{t}^{0}
$$

Although the ambiguity functions are heterogeneous, we note that $m_{t}^{X^{i}}$ is not dependent on $X_{t}^{i}$, so that the aggregated mean process is

$$
d m_{t}^{X}=-[1-\bar{\phi}] m_{t}^{Y} d t+\rho \sigma d W_{t}^{0}
$$

where $\bar{\phi}=\mathbb{E}\left[\phi_{i}\right]$, due to the symmetry in the control with respect to $m_{t}-X_{t}^{i}$.

For the remainder of the section, we make the ansatz

$$
Y_{t}=-\psi_{t}\left(m_{t}-X_{t}\right)
$$

Differentiating the ansatz, we obtain

$$
\begin{aligned}
d Y_{t}= & -\dot{\psi}_{t}\left(m_{t}-X_{t}\right) d t-\psi_{t} d\left(m_{t}-X_{t}\right) \\
= & {\left[-\dot{\psi}_{t}+\psi_{t}\left(a+q+\psi_{t}\left[1-\phi_{i}\right]\right)\right]\left(m_{t}-X_{t}\right) d t } \\
& +\psi_{t}^{2}[1-\bar{\phi}] m_{t}^{Y} d t+\psi_{t} \sigma \sqrt{1-\rho^{2}} d W_{t}
\end{aligned}
$$

Alternately, by plugging in the ansatz into the FBSDE, we obtain

$$
d Y_{t}=\left[-(a+q) \psi_{t}+\left(\epsilon-q^{2}\right)\right]\left(m_{t}-X_{t}\right) d t+Z_{t}^{0} d W_{t}^{0}+Z_{t} d W_{t}
$$

Comparing the forms for $d Y_{t}$, we get that $Z_{t}^{0}=0 \Longrightarrow m_{t}^{Y}=0$ and $\psi_{t}$ satisfies the following ODE

$$
\dot{\psi}_{t}=2(a+q) \psi_{t}+\left[1-\phi_{i}\right] \psi_{t}^{2}-\left(\epsilon-q^{2}\right)
$$

with terminal condition $\psi_{T}=c$.

If $\phi_{i}<1$ then the ODE admits a closed-form solution

$$
\psi(t)=\frac{-\left(\epsilon-q^{2}\right)\left(e^{\left(\delta^{+}-\delta^{-}\right)(T-t)}-1\right)-c\left(\delta^{+} e^{\left(\delta^{+}-\delta^{-}\right)(T-t)}-\delta^{-}\right)}{\left(\delta^{-} e^{\left(\delta^{+}-\delta^{-}\right)(T-t)}-\delta^{+}\right)-c\left(1-\phi_{i}\right)\left(e^{\left(\delta^{+}-\delta^{-}\right)(T-t)}-1\right)}
$$

where

$$
\delta^{ \pm}=-(a+q) \pm \sqrt{R}
$$

and

$$
R=(a+q)^{2}+\left(\epsilon-q^{2}\right)\left(1-\phi_{i}\right)
$$




\section{C Verification Theorem}

The verification for a mean-field game with ambiguity aversion is two-fold. First, we must verify that the ambiguity averse measure maximizes the expected value of the cost functional given the control $\alpha_{i}$. Then we must verify that the ambiguity averse agent's control minimizes the cost functional under the ambiguity averse measure. The arguments used are standard in the theory of optimal control, but are provided below for completeness.

We first consider the terminal cost $g\left(X_{T}\right)$. In the stochastic control problem we in fact solved $\mathbb{E}\left[\inf _{\alpha} \sup _{\eta} U(\alpha)\right]$

Holding $\alpha_{i} \in \mathcal{A}$ to a fixed adapted strategy, we write

$$
J(\alpha, \hat{\eta})-J(\alpha, \eta)=\mathbb{E}\left[\int_{0}^{T} f\left(t, \mu_{t}, \hat{X}_{t}, \alpha_{t}\right)-f\left(t, \mu_{t}, X_{t}, \alpha_{t}\right) d t+g\left(\hat{X}_{T}\right)-g\left(X_{T}\right)\right]
$$

where it is understood that $\hat{X}_{t}$ depends on an arbitrary control $\alpha_{t}$ and the optimal control for $\eta_{t}$.

For the remainder of the proof, we will use the notation $\hat{Y}_{t}=Y_{t}\left(\hat{X}_{t}\right), Y_{t}=Y_{t}\left(X_{t}\right)$.

Under the assumption of convexity, we have that

$$
g\left(\hat{X}_{T}\right)-g\left(X_{T}\right) \geq\left(\hat{X}_{T}-X_{T}\right) Y_{T}=\int_{0}^{T}\left(\hat{X}_{t}-X_{t}\right) d Y_{t}+\int_{0}^{T}\left(d \hat{X}_{t}-d X_{t}\right) Y_{t}
$$

Furthermore, we have

$$
\begin{aligned}
& \mathbb{E}\left[\int_{0}^{T} f\left(t, \mu_{t}, \hat{X}_{t}, \hat{\alpha}_{t}\right)-f\left(t, \mu_{t}, X_{t}, \hat{\alpha}_{t}\right) d t-\frac{1}{2 \phi_{i}} \int_{0}^{T}\left(\hat{\eta}^{2}-\eta^{2}\right)(t) d t\right] \\
& =\mathbb{E}\left[\int_{0}^{T} \mathcal{H}\left(t, \hat{X}_{t}, \widehat{\alpha}, \hat{Y}_{t}, \hat{Z}_{t}, \widehat{\eta}_{t}\right)-\mathcal{H}\left(t, X_{t}, \widehat{\alpha}, Y_{t}, \hat{Z}_{t}, \eta_{t}\right) d t-\int_{0}^{T}\left(d \hat{X}_{t} \hat{Y}_{t}-d X_{t} Y_{t}\right)\right] \\
& =\mathbb{E}\left[\int_{0}^{T} \mathcal{H}\left(t, \hat{X}_{t}, \widehat{\alpha}, \hat{Y}_{t}, \hat{Z}_{t}, \widehat{\eta}_{t}\right)-\mathcal{H}\left(t, X_{t}, \widehat{\alpha}, Y_{t}, \hat{Z}_{t}, \eta_{t}\right) d t\right. \\
& \left.\quad-\int_{0}^{T}\left(b\left(t, \hat{X}_{t}, \hat{Y}_{t}, \hat{\eta}_{t}\right)-b\left(t, X_{t}, Y_{t}, \eta_{t}\right)\right) Y_{t} d t-\int_{0}^{T} b\left(t, \hat{X}_{t}, \hat{Y}_{t}, \hat{\eta}_{t}\right)\left(\hat{Y}_{t}-Y_{t}\right) d t\right]
\end{aligned}
$$

where we take advantage of the fact that

$$
\mathcal{H}\left(t, X_{t}, \widehat{\alpha}, Y_{t}, \hat{Z}_{t}, \eta_{t}\right)-\mathcal{H}\left(t, X_{t}, \widehat{\alpha}, Y_{t}, Z_{t}, \eta_{t}\right)=\sigma^{T}\left(\hat{Z}_{t}-Z_{t}\right)
$$


which is not affected by the fact that the Brownian motions are correlated since the volatility process $\sigma$ is uncontrolled.

We now obtain independent bounds for three portions of this problem.

We have that

$\mathbb{E}\left[\int_{0}^{T} \mathcal{H}\left(t, \hat{X}_{t}, \widehat{\alpha}, Y_{t}, Z_{t}, \eta_{t}\right)-\mathcal{H}\left(t, X_{t}, \widehat{\alpha}, Y_{t}, Z_{t}, \eta_{t}\right) d t-\int_{0}^{T}\left(\hat{X}_{t}-X_{t}\right) \partial_{x} \mathcal{H}\left(t, X_{t}, \widehat{\alpha}, Y_{t}, Z_{t}, \eta_{t}\right) d t\right] \geq 0$

by convexity. In addition, we have

$\mathbb{E}\left[\int_{0}^{T} \mathcal{H}\left(t, \hat{X}_{t}, \widehat{\alpha}, \hat{Y}_{t}, \hat{Z}_{t}, \hat{\eta}_{t}\right)-\mathcal{H}\left(t, \hat{X}_{t}, \widehat{\alpha}, Y_{t}, \hat{Z}_{t}, \eta_{t}\right) d t-\int_{0}^{T} \partial_{y} \mathcal{H}\left(t, \hat{X}_{t}, \widehat{\alpha}, \hat{Y}_{t}, \hat{\eta}_{t}\right)\left(\hat{Y}_{t}-Y_{t}\right) d t\right] \geq 0$

since $\mathcal{H}$ is concave in $\left(Y_{t}, \eta_{t}\right)$, so that

$\mathcal{H}\left(t, \hat{X}_{t}, \widehat{\alpha}, \hat{Y}_{t}, \hat{Z}_{t}, \hat{\eta}_{t}\right)-\mathcal{H}\left(t, \hat{X}_{t}, \widehat{\alpha}, Y_{t}, \hat{Z}_{t}, \eta_{t}\right) \geq \partial_{y} \mathcal{H}\left(t, \hat{X}_{t}, \widehat{\alpha}, \hat{Y}_{t}, \hat{Z}_{t}, \hat{\eta}_{t}\right)\left(\hat{Y}_{t}-Y_{t}\right)+\partial_{\eta} \mathcal{H}\left(t, \hat{X}_{t}, \widehat{\alpha}, \hat{Y}_{t}, \hat{Z}_{t}, \hat{\eta}_{t}\right)\left(\hat{\eta}_{t}-\right.$

and the latter term $\partial_{\eta} \mathcal{H}\left(t, \hat{X}_{t}, \widehat{\alpha}, \hat{Y}_{t}, \hat{\eta}_{t}\right)\left(\hat{\eta}_{t}-\eta_{t}\right) \geq 0$ Therefore,

$$
\begin{aligned}
J\left(\hat{\alpha}_{t}, \hat{\eta}_{t}\right)-J\left(\hat{\alpha}_{t}, \eta_{t}\right) \\
\geq \mathbb{E}\left[\int_{0}^{T} \mathcal{H}\left(t, \hat{X}_{t}, \hat{\alpha}_{t}, \hat{Y}_{t}, \hat{Z}_{t}, \hat{\eta}_{t}\right)-\mathcal{H}\left(t, X_{t}, \hat{\alpha}_{t}, Y_{t}, \hat{Z}_{t}, \eta_{t}\right) d t\right. \\
\left.\quad-\int_{0}^{T}\left(d \hat{X}_{t} \hat{Y}_{t}-d X_{t} Y_{t}\right)+\left(\hat{X}_{t}-X_{t}\right) d Y_{t}+\int_{0}^{T}\left(d \hat{X}_{t}-d X_{t}\right) Y_{t}\right] \geq 0
\end{aligned}
$$

and so $\eta_{t}$ achieves a maximum with respect to any admissible control $\alpha_{t}$.

A similar argument yields that, again by convexity of $\mathrm{g}$ and Ito's formula, comparing the optimal strategy $\hat{\alpha}_{t}$ with an arbitrary control $\alpha_{t}$, we get $J\left(\hat{\alpha}_{t}, \hat{\eta}_{t}\right) \leq J\left(\alpha_{t}, \hat{\eta}_{t}\right)$. The proof is provided for completeness. Under the assumption of convexity, we have that

$$
g\left(\hat{X}_{T}\right)-g\left(X_{T}\right) \leq\left(\hat{X}_{T}-X_{T}\right) \hat{Y}_{T}=\int_{0}^{T}\left(\hat{X}_{t}-X_{t}\right) d \hat{Y}_{t}+\int_{0}^{T}\left(d \hat{X}_{t}-d X_{t}\right) \hat{Y}_{t}
$$

Furthermore, we have

$$
\begin{aligned}
& \mathbb{E}\left[\int_{0}^{T} f\left(t, \mu_{t}, \hat{X}_{t}, \hat{\alpha}_{t}\right)-f\left(t, \mu_{t}, X_{t}, \alpha_{t}\right) d t-\frac{1}{2 \phi_{i}} \int_{0}^{T}\left(\hat{\eta}^{2}-\eta^{2}\right)(t) d t\right] \\
& =\mathbb{E}\left[\int_{0}^{T} \mathcal{H}\left(t, \hat{X}_{t}, \hat{\alpha}_{t}, \hat{Y}_{t}, \hat{Z}_{t}, \widehat{\eta}\right)-\mathcal{H}\left(t, X_{t}, \alpha_{t}, \hat{Y}_{t}, \hat{Z}_{t}, \widehat{\eta}\right) d t-\int_{0}^{T}\left(d \hat{X}_{t}-d X_{t}\right) \hat{Y}_{t}\right]
\end{aligned}
$$


and so again, adding up the bounds for $\mathrm{f}$ and $\mathrm{g}$ yields

$$
\begin{aligned}
& J\left(\hat{\alpha}_{t}, \hat{\eta}_{t}\right)-J\left(\alpha_{t}, \hat{\eta}_{t}\right) \\
& \quad \leq \mathbb{E}\left[\int_{0}^{T} \mathcal{H}\left(t, \hat{X}_{t}, \hat{\alpha}_{t}, \hat{Y}_{t}, \hat{Z}_{t}, \widehat{\eta}\right)-\mathcal{H}\left(t, X_{t}, \alpha_{t}, \hat{Y}_{t}, \hat{Z}_{t}, \widehat{\eta}\right) d t+\int_{0}^{t}\left(\hat{X}_{t}-X_{t}\right) d \hat{Y}_{t}\right] \\
& \quad \leq \mathbb{E}\left[\int_{0}^{T} \mathcal{H}\left(t, \hat{X}_{t}, \hat{\alpha}_{t}, \hat{Y}_{t}, \hat{Z}_{t}, \widehat{\eta}\right)-\mathcal{H}\left(t, X_{t}, \alpha_{t}, \hat{Y}_{t}, \hat{Z}_{t}, \widehat{\eta}\right) d t-\int_{0}^{t}\left(\hat{X}_{t}-X_{t}\right) \partial_{x} \mathcal{H}\left(t, \hat{X}_{t}, \hat{\alpha}_{t}, \hat{Y}_{t}, \hat{Z}_{t}, \hat{\eta}\right)\right] \leq 0
\end{aligned}
$$

by convexity of $\mathcal{H}$ with respect to $(x, \alpha)$

The reader will note that this proof does not conclude that $\hat{\eta}$ is optimal in the stochastic control sense. In fact, $\hat{\eta}$ is optimal conditional on $\hat{\alpha}$ and vice versa. Therefore, $(\hat{\eta}, \hat{\alpha})$ is a Nash equilibrium. We also have that this yields a saddle point in the sense that

$$
J(\hat{\alpha}, \eta) \leq J(\hat{\alpha}, \hat{\eta}) \leq J(\alpha, \hat{\eta})
$$

Now the only point left to show is the that minimax point is achieved.

Assume there is another optimal strategy $(\tilde{\alpha}, \tilde{\eta})$ such that $J(\tilde{\alpha}, \tilde{\eta}(\tilde{\alpha}))=\min _{\alpha \in A} \max _{\eta \in E} J(\alpha, \eta)$ Then we have that

$$
J(\tilde{\alpha}, \tilde{\eta}(\tilde{\alpha})) \geq J(\tilde{\alpha}, \hat{\eta}) \geq J(\hat{\alpha}, \hat{\eta}) \geq J(\hat{\alpha}, \tilde{\eta}(\hat{\alpha})) \geq J(\tilde{\alpha}, \tilde{\eta}(\tilde{\alpha}))
$$

Therefore, any Nash equilibrium establishes a unique value for the game, and therefore

$$
J(\hat{\alpha}, \hat{\eta})=\min _{\alpha \in \mathcal{A}} \max _{\eta \in \mathcal{E}} J(\alpha, \eta)=\max _{\eta \in \mathcal{E}} \min _{\alpha \in \mathcal{A}} J(\alpha, \eta)
$$

\section{D Default}

Recall that the aggregate log-reserve moves according to the dynamics

$$
\frac{1}{N} \sum_{i=1}^{N} X_{t}^{i}=\rho W_{t}^{0}+\frac{1}{N} \sum_{i=1}^{N} \sqrt{1-\rho^{2}} W_{t}^{i}
$$


Therefore, the dynamics of the bank reserve for an individual bank can be written as

$$
\begin{aligned}
d X_{t}^{i} & =\left(a+q+\psi_{t}\right)\left(\bar{X}_{t}-X_{t}^{i}\right) d t+\rho d W_{t}^{0}+\sqrt{1-\rho^{2}} d W_{t}^{i} \\
& =-\left(a+q+\psi_{t}\right) X_{t}^{i}+\rho\left(a+q+\frac{\psi_{t}}{1-\phi_{a} \sigma^{2}}\right) W_{t}^{0} d t+\frac{\sqrt{1-\rho^{2}}}{N} \sum_{k=1}^{N}\left(a+q+\psi_{t}\right) W_{t}^{i} d t \\
& +\rho d W_{t}^{0}+\sqrt{1-\rho^{2}} d W_{t}^{i}
\end{aligned}
$$

by introducing an integrating factor

$$
Y_{t}^{i}=X_{t}^{i} e^{(a+q) t+\int_{0}^{t} \psi_{u} d u}
$$

we obtain

$$
\begin{aligned}
d Y_{t}^{i} & =\left(a+q+\frac{\psi_{t}}{1-\phi_{a} \sigma^{2}}\right) Y_{t}^{i}+e^{(a+q) t+\int_{0}^{t} \psi_{u} d u} d X_{t} \\
& =e^{(a+q) t+\int_{0}^{t} \psi_{u} d u}\left(\rho\left(a+q+\psi_{t}\right) W_{t}^{0} d t+\frac{\sqrt{1-\rho^{2}}}{N} \sum_{k=1}^{N}\left(a+q+\psi_{t}\right) W_{t}^{k} d t\right. \\
& \left.+\rho d W_{t}^{0}+\sqrt{1-\rho^{2}} d W_{t}^{i}\right)
\end{aligned}
$$

and therefore, by integrating $X_{t}^{i}$ has the form

$$
\begin{aligned}
X_{t}^{i} & =X_{0}^{i} e^{-(a+q) t-\int_{0}^{t} \psi_{u} d u}+\rho \int_{0}^{t} e^{-(a+q)(t-v)-\int_{v}^{t} \psi_{u} d u}\left(1+a+q+\psi_{v}\right) W_{v}^{0} d v \\
& +\frac{\sqrt{1-\rho^{2}}}{N} \sum_{k=1}^{N} \int_{0}^{t} e^{-(a+q)(t-v)-\int_{v}^{t} \psi_{u} d u}\left(N \delta_{i, k}+a+q+\psi_{v}\right) W_{v}^{k} d v
\end{aligned}
$$


and performing a change in order of integration (since $\psi_{t} \in C_{b}(\mathbb{R})$ )

$$
\begin{aligned}
X_{t}^{i}= & X_{0}^{i} e^{-(a+q) t-\int_{0}^{t} \psi_{u} d u}+\rho \int_{0}^{t} \int_{s}^{t} e^{-(a+q)(t-v)-\int_{v}^{t} \psi_{u} d u}\left(a+q+\psi_{v}\right) d v d W_{s}^{0} \\
& +\frac{\sqrt{1-\rho^{2}}}{N} \sum_{k=1}^{N} \int_{0}^{t} \int_{s}^{t} e^{-(a+q)(t-v)-\int_{v}^{t} \psi_{u} d u}\left(a+q+\psi_{v}\right) d v d W_{s}^{k} \\
& +\rho \int_{0}^{t} e^{-(a+q)(t-v)-\int_{v}^{t} \psi_{u} d u} d W_{v}^{0}+\sqrt{1-\rho^{2}} \int_{0}^{t} e^{-(a+q)(t-v)-\int_{v}^{t} \psi_{u} d u} d W_{v}^{i} \\
= & X_{0}^{i} e^{-(a+q) t-\int_{0}^{t} \psi_{u} d u} \\
& +\rho \int_{0}^{t}\left(1-e^{-(a+q)(t-s)-\int_{s}^{t} \psi_{u} d u}\right) d W_{s}^{0}+\frac{\sqrt{1-\rho^{2}}}{N} \sum_{k=1}^{N} \int_{0}^{t}\left(1-e^{-(a+q)(t-s)-\int_{s}^{t} \psi_{u} d u}\right) d W_{s}^{k} \\
& +\rho \int_{0}^{t} e^{-(a+q)(t-v)-\int_{v}^{t} \psi_{u} d u} d W_{v}^{0}+\sqrt{1-\rho^{2}} \int_{0}^{t} e^{-(a+q)(t-v)-\int_{v}^{t} \psi_{u} d u} d W_{v}^{i} \\
= & X_{0}^{i} e^{-(a+q) t-\int_{0}^{t} \psi_{u} d u} \\
& +\rho d W_{t}+\frac{\sqrt{1-\rho^{2}}}{N} \sum_{k=1, k \neq i}^{N} \int_{0}^{t}\left(1-e^{-(a+q)(t-s)-\int_{s}^{t} \psi_{u} d u}\right) d W_{s}^{k} \\
& +\frac{\sqrt{1-\rho^{2}}}{N} \int_{0}^{t}\left(1-e^{-(a+q)(t-s)-\int_{s}^{t} \psi_{u} d u}+N e^{-(a+q)(t-s)-\int_{s}^{t} \psi_{u} d u}\right) d W_{s}^{i}
\end{aligned}
$$

And, denoting $\tilde{\psi}_{t}=\int_{0}^{t}\left(a+q+\psi_{s}\right) d s$, we can reduce this expression to

$$
\begin{aligned}
X_{t}^{i}= & X_{0}^{i} e^{-\tilde{\psi}_{t}}+\rho e^{-\tilde{\psi}_{t}} \int_{0}^{t} e^{\tilde{\psi}_{s}}\left(1+\int_{s}^{t} e^{-\tilde{\psi}_{s}+\tilde{\psi}_{u} d u}\right) d W_{s}^{0} \\
& +\frac{\sqrt{1-\rho^{2}}}{N} \sum_{k=1}^{N} e^{-\tilde{\psi}_{t}} \int_{0}^{t} e^{\tilde{\psi}_{s}}\left(1+N \delta_{i, k} \int_{s}^{t} e^{-\tilde{\psi}_{s}+\tilde{\psi}_{u} d u}\right) d W_{s}^{k}
\end{aligned}
$$

Therefore, by Ito's Isometry, we get that the distribution of $X_{t}^{i}$ is identical to that of an Ornstein-Uhlenbeck process with time-varying drift and diffusion terms.

$$
d \tilde{X}_{t}^{i}=-\gamma(t) \tilde{X}_{t}^{i} d t+\sigma(t) d W_{t}^{i}
$$

and since $\gamma(t), \sigma(t)$ are continuous, the stopping times associated with this process has the same properties as an Ornstein-Uhlenbeck process defined above. 
The default probability is

$$
\mathbb{P}\left(\min _{0 \leq t \leq T} X_{t}^{i}<D\right)
$$

which we can find in semi-analytic form.

We define a stopping time $\tau=\min \left\{t \mid X_{t}^{i}=D\right\}$ and a function

$$
g(x, t)=\mathbb{E}\left[1_{\tau \geq T}\left(X_{t}^{i}\right) \mid X_{t}^{i}=x\right]
$$

The PDE that $g$ satisfies is

$$
\left(\partial_{t}+\frac{\beta(t)^{2}}{2} \partial_{x x}-\gamma(t) X_{t}^{i} \partial_{x}\right) g=0
$$

with boundary conditions

$$
\begin{aligned}
& g(x, T)=1_{\{x<D\}} \\
& g(D, t)=1
\end{aligned}
$$

where

$$
\begin{aligned}
\gamma_{t}=a & +q+\psi_{t} \\
\beta_{t}^{2}=\rho^{2} & +(N-1) \frac{1-\rho^{2}}{N^{2}} \int_{0}^{t}\left(1-e^{-(a+q)(t-s)-\int_{s}^{t} \psi_{u} d u}\right)^{2} d s \\
& +\frac{1-\rho^{2}}{N^{2}} \int_{0}^{t}\left(1-e^{-(a+q)(t-s)-\int_{s}^{t} \psi_{u} d u}+N e^{-(a+q)(t-s)-\int_{s}^{t} \psi_{u} d u}\right)^{2} d s
\end{aligned}
$$

which is similar to the PDE of a digital down-and-in barrier option.

Theorem 3.D.1. For any choice in parameters $a, q, c, \epsilon, \bar{\phi}, T>0$, there is a $N_{0}$ such that for any $N>N_{0}$, if $\bar{\phi}>\phi_{a}>\phi_{b}>0$ then simultaneously $\beta_{t, a}<\beta_{t, b}$ and $\gamma_{t, a}>\gamma_{t, b}$

Proof. It is trivial to show that $\psi_{t}$ is an increasing function of $\phi$ based on the ODE form of $\psi_{t}$.

To show that $\beta_{t}^{2}$ is a monotonic decreasing function, we replace $e^{-(a+q)(t-s)-\int_{s}^{t} \psi_{u} d u}$ with $\mathrm{x}$ and show it that the sum of the integrands are monotonically increasing with respect to $\mathrm{x}$ for any $x \in[0,1]$, since $e^{-(a+q)(t-s)-\int_{s}^{t} \psi_{u} d u}$ is monotonically decreasing with respect to $\phi$. 
Then the sum of the integrands can be written as

$$
f(x)=(N-1) \frac{1-\rho^{2}}{N^{2}}(1-x)^{2}+\frac{1-\rho^{2}}{N^{2}}(1+(N-1) x)^{2}
$$

Taking derivatives with respect to $\mathrm{x}$, we find that

$$
\begin{aligned}
\frac{\partial f}{\partial x} & =(N-1) \frac{1-\rho^{2}}{N^{2}}(x-1)+(N-1) \frac{1-\rho^{2}}{N^{2}}(1+(N-1) x) \\
& =x\left(1-\rho^{2}\right)\left(\frac{N-1}{N^{2}}+\left(\frac{N-1}{N}\right)^{2}\right)-2\left(1-\rho^{2}\right)\left(\frac{N-1}{N^{2}}\right)
\end{aligned}
$$

so that as long as $\phi$ is in some bounded set $\mathcal{P}$, then $x$ is bounded below by some $x_{0}>0$. Therefore, as long as

$$
\frac{x_{0}\left(\frac{N-1}{N^{2}}+\left(\frac{N-1}{N}\right)^{2}\right)}{2\left(\frac{N-1}{N^{2}}\right)}>1
$$

holds for sufficiently large $\mathrm{N}$, then $\beta_{t}^{2}$ is monotonically decreasing with respect to $\phi \in \mathcal{P}$.

Note that the form of this PDE also shows that ambiguity aversion will help only if $\rho<1$, as when $\rho=1$, then $m_{0}, X_{0}$ co-move in the same direction at the same rate, so the problem essentially becomes a deterministic optimization problem.

\section{D.1 Comparison for PDE}

Theorem 3.D.2. Given $N$ from above, if $D<X_{0}^{i}<\bar{X}_{0}$ then $\phi_{a}<\phi_{b} \Longrightarrow P\left(X_{T}<\right.$ $\left.D \mid \phi_{a}\right)>P\left(X_{T}<D \mid \phi_{b}\right)$

Proof. As the process is mean-reverting, we can apply two comparison theorems to obtain the result.

Formally, if we have two stochastic processes $X_{t}^{1}, X_{t}^{2}$ with dynamics

$$
\begin{aligned}
& d X_{t}^{1}=-b^{1}(t) X_{t}^{1} d t+\sigma^{1}(t) d W_{t} \\
& d X_{t}^{2}=-b^{2}(t) X_{t}^{2} d t+\sigma^{2}(t) d W_{t}
\end{aligned}
$$

and $D<X_{0}^{1}=X_{0}^{2}<0$ then if $b^{1}(t)>b^{2}(t), \sigma^{1}(t)=\sigma^{2}(t)$ then $P\left(X_{T}^{1}<D\right)<P\left(X_{T}^{2}<\right.$ $D)$.

Similarly, if $b^{1}(t)=b^{2}(t), \sigma^{1}(t)<\sigma^{2}(t)$ then $P\left(X_{T}^{1}<D\right)<P\left(X_{T}^{2}<D\right)$.

Therefore, as $b(t), \sigma(t)$ are monotonic functions of $\phi$ (respectively increasing and decreasing), we assert that if $D<X_{0}^{i}<\bar{X}_{0}$ then $\phi_{a}<\phi_{b} \Longrightarrow P\left(X_{T}<D \mid \phi_{a}\right)>P\left(X_{T}<D \mid \phi_{b}\right)$ 


\section{Chapter 4}

\section{Mean-Field Games and Ambiguity Aversion}

\subsection{Introduction}

The first questions of existence and uniqueness of mean-field games was addressed by Lasry and Lions (2007a) and Huang et al. (2006). One of the first papers to consider robustness in mean-field games was Bauso et al. (2012), and more recently, extending to the linear-quadratic framework, Moon and Başar (2017). However, in both of these papers, there is no mean-field term in the dynamics, which allows them to use the result from Lasry and Lions (2007a) to establish existence and uniqueness. In this paper, we propose to use the framework of Carmona and Delarue (2013) for a general existence/uniqueness result for robust convex-concave mean-field games. Afterwards, we look at the linear-quadratic framework with a mean-field term in the dynamics, and characterize its solution as well as the conditions for existence, uniqueness and an $\epsilon$-Nash equilibrium to hold in section 4.5.

In Carmona and Delarue (2013), the players' dynamics were given by

$$
d X_{t}^{i}=b^{i}\left(t, X^{i}, \mu_{t}, \alpha^{i}\right) d t+\sigma\left(t, X_{t}^{i}\right) d W_{t}^{i}
$$

and the respective cost functions were

$$
J^{i}\left(\alpha^{i}\right)=\mathbb{E}\left[\int_{0}^{T} f^{i}\left(t, X^{i}, \mu^{i}, \alpha^{i}\right) d t+g^{i}\left(T, X^{i}\right)\right]
$$

where the goal of player $i$ is to minimize her cost through her control $\alpha^{i}$, and $\mu_{t}$ is the mean-field measure flow which describes the distribution of player states at time $t$. 
We now extend this setup to incorporate model uncertainty. In particular, we will look at a mean-field game where all the players are ambiguity averse.

\subsubsection{N-player game}

To begin, for $1 \leq i \leq N$, we let $\boldsymbol{W}_{t}^{i}$ be an $m$-dimensional uncorrelated standard Brownian motion defined on the filtered probability space $\left(\Omega, \mathcal{F}^{i},\left\{\mathcal{F}_{t}^{i}\right\}_{t \geq 0}, \mathbb{P}\right)$, where $\left\{\mathcal{F}_{t}^{i}\right\}_{t \geq 0}$ is the $\mathbb{P}$-augmentation of the natural filtration generated by $\boldsymbol{W}_{t}^{i}$. We search for optimal controls $\alpha_{t}^{i}, \eta_{t}^{i}$ within the admissible sets $\mathcal{A}, \mathcal{E} \subset \mathbb{R}^{k}$ respectively. The private states of player $i$ are denoted as $U_{t}^{i} \in \mathbb{R}^{d}$. The drift and volatility processes respectively are $b^{i}:[0, T] \times \mathbb{R}^{d} \times \mathcal{P}\left(\mathbb{R}^{d}\right) \times \mathcal{A} \times \mathcal{E} \rightarrow \mathbb{R}^{d}$ and $\sigma^{i}:[0, T] \rightarrow \mathbb{R}^{d \times m}$. We define the empirical distribution of the players' states $\left(U_{t}^{1}, \ldots, U_{t}^{N}\right)$ are

$$
\bar{\nu}_{t}=\frac{1}{N} \sum_{i=1}^{N} \delta_{U_{t}^{i}}
$$

The stochastic differential equation for each player's state is then

$$
d U_{t}^{i}=b^{i}\left(t, U_{t}^{i}, \bar{\nu}_{t}, \alpha_{t}^{i}, \eta_{t}^{i}\right) d t+\sigma^{i}(t) d W_{t}^{i}
$$

The strategies $\alpha_{t}^{i}, \eta_{t}$ are chosen such that

$$
\mathbb{E}\left[\int_{0}^{T}\left|\alpha_{t}^{i}\right|^{2} d t\right]<\infty, \quad \mathbb{E}\left[\int_{0}^{T}\left|\eta_{t}^{i}\right|^{2} d t\right]<\infty
$$

and minimize/maximize the cost function

$$
J^{i}(\alpha, \eta)=\mathbb{E}\left[\int_{0}^{T} f^{i}\left(t, U_{t}^{i}, \bar{\nu}_{t}, \alpha_{t}^{i}, \eta_{t}^{i}\right) d t+g^{i}\left(U_{T}^{i}, \bar{\nu}_{T}\right)\right]
$$

where $f^{i}:[0, T] \times \mathbb{R}^{d} \times \mathcal{P}\left(\mathbb{R}^{d}\right) \times \mathcal{A} \times \mathcal{E} \rightarrow \mathbb{R}$ and $g^{i}: \mathbb{R}^{d} \times \mathcal{P}\left(\mathbb{R}^{d}\right) \rightarrow \mathbb{R}$. In particular, the players wish to find a control $\widehat{\alpha}_{t}^{i}$ such that

$$
J^{i}(\widehat{\alpha}, \widehat{\eta})=\inf _{\alpha_{t}^{i} \in \mathcal{A}} \sup _{\eta_{t}^{i} \in \mathcal{E}} \mathbb{E}\left[\int_{0}^{T} f^{i}\left(t, U_{t}^{i}, \bar{\nu}_{t}, \alpha_{t}^{i}, \eta_{t}^{i}\right) d t+g^{i}\left(U_{T}^{i}, \bar{\nu}_{T}\right)\right]
$$

\subsubsection{Mean-Field Game}

We can introduce the mean-field limit of the game with ambiguity averse players, with the frozen measure flow $\mu_{t}$ replacing the empirical distribution $\bar{\nu}_{t}$, in which case the players' 
dynamics become

$$
d X_{t}^{i}=b^{i}\left(t, X^{i}, \mu_{t}, \alpha^{i}, \eta^{i}\right) d t+\sigma\left(t, X_{t}^{i}\right) d W_{t}^{i}
$$

with cost functions

$$
J^{i}\left(\alpha^{i}, \eta^{i}\right)=\mathbb{E}\left[\int_{0}^{T} f^{i}\left(t, X^{i}, \mu_{t}, \alpha^{i}, \eta^{i}\right) d t+g^{i}\left(X_{T}^{i}, \mu_{T}\right)\right]
$$

Note that by having a frozen measure flow $\mu_{t}$, the minimax cost for a representative agent can be viewed as a standard optimization problem.

The objective in this case is to minimize the worst-case misspecification of the model, so player $i$ wishes to find a strategy $\hat{\alpha}^{i}$ such that the the following cost function is minimized

$$
J\left(\alpha^{i}, \widehat{\eta}^{i}\right)=\sup _{\eta_{t}^{i} \in \mathcal{E}} \mathbb{E}\left[\int_{0}^{T} f^{i}\left(t, X^{i}, \mu_{t}, \alpha^{i}, \eta^{i}\right) d t+g^{i}\left(X_{T}^{i}, \mu_{T}\right)\right]
$$

A simple class of models which fall under this framework would be the linear-quadratic model, in which case we would assume that the drift is linear and the cost is quadratic and convex in $(x, \alpha)$, and quadratic and concave in $\eta^{i}$.

\subsubsection{Optimizing via the Hamiltonian}

We introduce the Hamiltonian

$$
\mathcal{H}(t, x, \mu, y, \alpha, \eta)=\langle b(t, x, \mu, \alpha, \eta), y\rangle+f(t, x, \mu, \alpha, \eta)
$$

The purpose is to find the minimax of the Hamiltonian, and determine sufficient conditions under which the minimax controls for the Hamiltonian exist, and will result in the minimax controls for the mean-field game.

To this end, we assume that there is a pair $(\widehat{\alpha}, \widehat{\eta})$ such that

$$
\mathcal{H}\left(t, \hat{X}_{t}, \hat{\alpha}_{t}, \hat{Y}_{t}, \hat{Z}_{t}, \hat{\eta}_{t}\right)=\min _{\alpha \in A} \max _{\eta \in E} \mathcal{H}\left(t, \hat{X}_{t}, \alpha, \hat{Y}_{t}, \hat{Z}_{t}, \eta\right)
$$

From 4.2.1 we will see that under certain conditions, this equilibrium gives a minimax value for the game.

Here we follow the notation introduced by Carmona and Delarue (2013) and denote $\mathcal{P}_{p}(E)$ to be the set of probability measures of order $\mathrm{p}$, so that

$$
M_{p, E}(\mu)=\left(\int_{E}\|x\|_{E}^{p} d \mu(x)\right)^{1 / p}<\infty
$$


where $\mathrm{E}$ is a separable Banach space and $\mu \in \mathcal{P}_{p}(E)$.

Assumption 4.1.1. The function $[0, T] \in t \rightarrow b_{1}(t, x, \mu)$ is affine in $x, \alpha$ and $\eta$. ie $b(t, x, \mu, \alpha, \eta)=b_{0}(t, \mu)+b_{1}(t) x+b_{2}(t) \alpha+b_{3}(t) \eta$

Assumption 4.1.2. The cost function satisfies the following inequality

$$
f\left(t, x^{\prime}, \mu, \alpha^{\prime}, \eta\right)-f(t, x, \mu, \alpha, \eta)-\left\langle\left(x^{\prime}-x, \alpha^{\prime}-\alpha\right), \partial_{x, \alpha} f(t, x, \mu, \alpha, \eta)\right\rangle \geq \lambda\left(\left|\alpha^{\prime}-\alpha\right|^{2}\right)
$$

This is the standard definition for the cost function to be jointly convex in $(x, \alpha)$.

Given that $\eta$ is maximizing the cost function, we can see that having $\eta^{\prime}=\eta$ gives us the usual definitions for joint convexity.

Assumption 4.1.3. The function $x \rightarrow g(x, \mu)$ is locally bounded, once continuously differentiable, convex and has a Lipschitz continuous first order derivative.

Assumption 4.1.4. For the minimizer $\widehat{\alpha}(t, x, \mu, y)$ of the Hamiltonian in feedback form, we have that the Hamiltonian is jointly concave in the variables $(y, \eta)$.

$\mathcal{H}(t, x, \mu, \widehat{\alpha}, y, \eta)-\mathcal{H}\left(t, x, \mu, \widehat{\alpha}^{\prime}, y^{\prime}, \eta^{\prime}\right)-\left\langle\left(y-y^{\prime}, \eta-\eta^{\prime}\right), \partial_{y, \eta} \mathcal{H}(t, x, \mu, \widehat{\alpha}, y, \eta)\right\rangle \geq \lambda_{\eta}\left(\left|\eta^{\prime}-\eta\right|^{2}\right)$

where $\widehat{\alpha}=\widehat{\alpha}(t, x, \mu, y)$ and $\widehat{\alpha}^{\prime}=\widehat{\alpha}\left(t, x, \mu, y^{\prime}\right)$.

Although this assumption looks strange at first, it is consistent with the assumptions for the verification theorem 4.2.1 to hold and allows the problem to have a well-defined control $\widehat{\eta}$ which maximizes the performance criteria. In the linear-quadratic case, the purpose of equation (4.8) is similar to the concept of disturbance attenuation in Bauso et al. (2016), as the optimal strategy for a player who is extremely ambiguity averse may not be admissible. Mathematically, a cost function which is not sufficiently concave may not have an attainable supremum. We will show in section 4.5 that this is a sharp bound for the linear-quadratic game.

Lemma 4.1.1. There exists a unique minimizer (resp maximizer) $\hat{\alpha}$ (resp $\hat{\eta}$ ) of $\mathcal{H}$. Moreover, $\hat{\alpha}, \hat{\eta}$ are Lipschitz-continuous with respect to $(x, y)$, uniformly in $(t, \mu)$

Proof. We have the inequality

$$
\begin{aligned}
\mathcal{H}(t, x, \mu, 0, y, \eta) & \geq \mathcal{H}(t, x, \mu, \widehat{\alpha}, y, \eta) \\
& \geq \mathcal{H}(t, x, \mu, 0, y, \eta)+\left\langle\hat{\alpha}, \partial_{\alpha} \mathcal{H}\right\rangle+\lambda|\hat{\alpha}|^{2}
\end{aligned}
$$


so that

$$
\hat{\alpha}(t, x, \mu, y) \leq \frac{1}{\lambda}\left(\left|\partial_{\alpha} f(t, x, \mu, 0, \eta)\right|+\left|b_{2}(t)\right| y\right)
$$

Similarly, we have that

$$
\hat{\eta}(t, x, \mu, y) \leq \frac{1}{\lambda_{\eta}}\left(\left|\partial_{\eta} f(t, x, \mu, \alpha, 0)\right|+\left|b_{3}(t)\right| y\right)
$$

These bounds help to control the growth rate of $\widehat{\alpha}, \widehat{\eta}$ in later proofs. The following three assumptions are regularity assumptions which allow us to show that there exists a unique equilibrium for the mean-field game.

Assumption 4.1.5. For all $0 \leq t \leq T, x, x^{\prime} \in \mathbb{R}^{d}, \alpha, \alpha^{\prime} \in \mathbb{R}^{K}, \mu, \mu^{\prime} \in \mathcal{P}_{2}\left(\mathbb{R}^{d}\right)$, we have

$$
\begin{aligned}
& \left|(f, g)\left(t, x^{\prime}, \mu^{\prime}, \alpha^{\prime}, \eta^{\prime}\right)-(f, g)(t, x, \mu, \alpha, \eta)\right| \\
& \quad \leq C\left[1+\left|\left(x^{\prime}, \alpha^{\prime}, \eta^{\prime}\right)\right|+|(x, \alpha, \eta)|+M_{2}(\mu)+M_{2}\left(\mu^{\prime}\right)\right]\left[\left|\left(x^{\prime}, \alpha^{\prime}, \eta^{\prime}\right)-(x, \alpha, \eta)\right|+W_{2}^{2}\left(\mu^{\prime}, \mu\right)\right]
\end{aligned}
$$

Furthermore, we have that $b_{0}, b_{1}, b_{2}$ are bounded by $C$ and

$$
\left|b_{0}\left(t, \mu^{\prime}\right)-b_{0}(t, \mu)\right| \leq C W_{2}\left(\mu, \mu^{\prime}\right)
$$

We note that a consequence of Assumption 4.1.5 is that there exists a $\lambda^{\prime} \in \mathbb{R}^{+}$such that

$f\left(t, x^{\prime}, \mu, \alpha^{\prime}, \eta^{\prime}\right)-f(t, x, \mu, \alpha, \eta)-\left\langle\left(x^{\prime}-x, \alpha^{\prime}-\alpha\right), \partial_{x, \alpha} f(t, x, \mu, \alpha, \eta)\right\rangle \geq \lambda\left(\left|\alpha^{\prime}-\alpha\right|^{2}\right)-\lambda^{\prime}\left(\left|\eta^{\prime}-\eta\right|^{2}\right)$

Assumption 4.1.6. For all $(t, x, \mu),\left|\partial_{\alpha} f(t, x, \mu, 0, \eta)\right|,\left|\partial_{\eta} f(t, x, \mu, \alpha, 0)\right| \leq C$

Assumption 4.1.7. For all $(t, x),\left\langle x, \partial_{x} f\left(t, 0, \delta_{x}, 0,0\right)\right\rangle \geq-C(1+|x|)$ and $\left\langle x, \partial_{x} g\left(0, \delta_{x}\right)\right\rangle \geq$ $-C(1+|x|)$

\subsection{Stochastic Nash-Pontryagin Principle}

We introduce a modification of the stochastic Pontryagin principle to deal with the minimization-maximization problem, as the standard stochastic maximum principle only provides a result for either minimization or maximization. A deterministic version is proven in Geering (2007), however the techniques used break down in the stochastic 
control setting. We provide a proof of the stochastic Nash-Pontryagin principle in our setting to establish the existence of a Nash equilibrium.

Theorem 4.2.1. [Stochastic Nash-Pontryagin Principle] Assume that there exists a solution $\hat{Y}_{t}, \hat{Z}_{t}$ to the BSDE

$$
d \hat{Y}_{t}=-\partial_{x} \widehat{\mathcal{H}}\left(t, \hat{X}_{t}, \hat{\alpha}_{t}, \hat{Y}_{t}, \hat{Z}_{t}, \hat{\eta}_{t}\right) d t+\hat{Z}_{t} d W_{t}, \quad Y_{T}=g\left(X_{T}\right)
$$

such that

$$
\widehat{\mathcal{H}}\left(t, \hat{X}_{t}, \hat{\alpha}_{t}, \hat{Y}_{t}, \hat{Z}_{t}, \hat{\eta}_{t}\right)=\min _{\alpha \in A} \max _{\eta \in E} \mathcal{H}\left(t, \hat{X}_{t}, \alpha, \hat{Y}_{t}, \hat{Z}_{t}, \eta\right)
$$

and

$$
\mathcal{H}\left(t, x, \alpha_{t}, \hat{y}, \hat{z}, \widehat{\eta}_{t}\right)=\max _{\eta \in \mathcal{B}} \mathcal{H}(t, x, \alpha, \hat{y}, \hat{z}, \widehat{\eta})
$$

is convex in $(\alpha, x)$ and

$$
\mathcal{H}\left(t, x, \hat{\alpha}_{t}(x, y, z), y, z, \eta_{t}\right)=\min _{\alpha \in A} \mathcal{H}(t, x, \alpha, y, z, \eta)
$$

is concave is $(y, \eta)$ and convex in $x$.

Given the mean-field-game measure flow $\mu_{t}$, the optimal strategy of the $i^{\text {th }}$ agent $\alpha^{i}(t)$ minimizes her cost function $J\left(\alpha_{i} ; \alpha_{-i}\right)$, so that for any strategy $\alpha_{i} \in \mathcal{A}$ and

$$
J\left(\alpha_{i}, \hat{\eta}^{i}\right) \geq J\left(\hat{\alpha}_{i}, \hat{\eta}^{i}\right), \quad \forall i
$$

and $\eta^{i}$ maximizes the cost function such that for any alternative strategy $\eta^{i} \in \mathcal{E}$, and for the optimal $\alpha^{i} \in \mathcal{A}$ we have

$$
J\left(\hat{\alpha}_{i}, \eta^{i}\right) \leq J\left(\hat{\alpha}_{i}, \hat{\eta}^{i}\right)
$$

and hence

$$
J(\hat{\alpha}, \hat{\eta})=\min _{\alpha \in A} \max _{\eta \in E} J(\alpha, \eta)=\max _{\eta \in E} \min _{\alpha \in A} J(\alpha, \eta)
$$

Proof. The verification for a mean-field game with ambiguity aversion is two-fold. First, we must verify that the ambiguity averse measure maximizes the expected value of the cost functional given the control $\alpha_{i}$. Then we must verify that the ambiguity averse agent's control minimizes the cost functional under the ambiguity averse measure. The arguments used are standard in the theory of optimal control, but are provided below for completeness.

We first consider the terminal cost $g\left(X_{T}\right)$. In the stochastic control problem we in fact solved $\mathbb{E}\left[\inf _{\alpha} \sup _{\eta} U(\alpha)\right]$ 
Holding $\alpha_{i} \in \mathcal{A}$ to a fixed adapted strategy, we write

$$
J(\alpha, \widehat{\eta})-J(\alpha, \widehat{\eta})=\mathbb{E}\left[\int_{0}^{T} f\left(t, \mu_{t}, \hat{X}_{t}, \alpha_{t}, \widehat{\eta}\right)-f\left(t, \mu_{t}, X_{t}, \alpha_{t}, \widehat{\eta}\right) d t+g\left(\hat{X}_{T}\right)-g\left(X_{T}\right)\right]
$$

where it is understood that $\hat{X}_{t}$ depends on an arbitrary control $\alpha_{t}$ and the optimal control for $\eta_{t}$.

For the remainder of the proof, we will use the notation $\hat{Y}_{t}=Y_{t}\left(\hat{X}_{t}\right), Y_{t}=Y_{t}\left(X_{t}\right)$.

Under the assumption of convexity, we have that

$$
g\left(\hat{X}_{T}\right)-g\left(X_{T}\right) \geq\left(\hat{X}_{T}-X_{T}\right) Y_{T}=\int_{0}^{T}\left(\hat{X}_{t}-X_{t}\right) d Y_{t}+\int_{0}^{T}\left(d \hat{X}_{t}-d X_{t}\right) Y_{t}
$$

Furthermore, we have

$$
\begin{aligned}
\mathbb{E} & {\left[\int_{0}^{T} f\left(t, \mu_{t}, \hat{X}_{t}, \hat{\alpha}_{t}, \hat{\eta}_{t}\right)-f\left(t, \mu_{t}, X_{t}, \hat{\alpha}_{t}, \widehat{\eta}_{t}\right) d t\right] } \\
=\mathbb{E} & {\left[\int_{0}^{T} \mathcal{H}\left(t, \hat{X}_{t}, \widehat{\alpha}, \hat{Y}_{t}, \hat{Z}_{t}, \widehat{\eta}_{t}\right)-\mathcal{H}\left(t, X_{t}, \widehat{\alpha}, Y_{t}, \hat{Z}_{t}, \eta_{t}\right) d t-\int_{0}^{T}\left(d \hat{X}_{t} \hat{Y}_{t}-d X_{t} Y_{t}\right)\right] } \\
=\mathbb{E} & {\left[\int_{0}^{T} \mathcal{H}\left(t, \hat{X}_{t}, \widehat{\alpha}, \hat{Y}_{t}, \hat{Z}_{t}, \widehat{\eta}_{t}\right)-\mathcal{H}\left(t, X_{t}, \widehat{\alpha}, Y_{t}, \hat{Z}_{t}, \eta_{t}\right) d t\right.} \\
& \left.\quad-\int_{0}^{T}\left(b\left(t, \hat{X}_{t}, \hat{Y}_{t}, \hat{\eta}_{t}\right)-b\left(t, X_{t}, Y_{t}, \eta_{t}\right)\right) Y_{t} d t-\int_{0}^{T} b\left(t, \hat{X}_{t}, \hat{Y}_{t}, \hat{\eta}_{t}\right)\left(\hat{Y}_{t}-Y_{t}\right) d t\right]
\end{aligned}
$$

where we take advantage of the fact that

$$
\mathcal{H}\left(t, X_{t}, \widehat{\alpha}, Y_{t}, \hat{Z}_{t}, \eta_{t}\right)-\mathcal{H}\left(t, X_{t}, \widehat{\alpha}, Y_{t}, Z_{t}, \eta_{t}\right)=\sigma^{T}\left(\hat{Z}_{t}-Z_{t}\right)
$$

We now obtain independent bounds for three portions of this problem.

We have that

$\mathbb{E}\left[\int_{0}^{T} \mathcal{H}\left(t, \hat{X}_{t}, \widehat{\alpha}, Y_{t}, Z_{t}, \eta_{t}\right)-\mathcal{H}\left(t, X_{t}, \widehat{\alpha}, Y_{t}, Z_{t}, \eta_{t}\right) d t-\int_{0}^{T}\left(\hat{X}_{t}-X_{t}\right) \partial_{x} \mathcal{H}\left(t, X_{t}, \widehat{\alpha}, Y_{t}, Z_{t}, \eta_{t}\right) d t\right] \geq 0$

by convexity.

In addition, we have

$$
\mathbb{E}\left[\int_{0}^{T} \mathcal{H}\left(t, \hat{X}_{t}, \widehat{\alpha}, \hat{Y}_{t}, \hat{Z}_{t}, \hat{\eta}_{t}\right)-\mathcal{H}\left(t, \hat{X}_{t}, \widehat{\alpha}, Y_{t}, \hat{Z}_{t}, \eta_{t}\right) d t-\int_{0}^{T} \partial_{y} \mathcal{H}\left(t, \hat{X}_{t}, \widehat{\alpha}, \hat{Y}_{t}, \hat{\eta}_{t}\right)\left(\hat{Y}_{t}-Y_{t}\right) d t\right] \geq 0
$$


since $\mathcal{H}$ is concave in $\left(Y_{t}, \eta_{t}\right)$, so that

$$
\begin{aligned}
& \mathcal{H}\left(t, \hat{X}_{t}, \widehat{\alpha}, \hat{Y}_{t}, \hat{Z}_{t}, \hat{\eta}_{t}\right)-\mathcal{H}\left(t, \hat{X}_{t}, \widehat{\alpha}, Y_{t}, \hat{Z}_{t}, \eta_{t}\right) \\
& \quad \geq \partial_{y} \mathcal{H}\left(t, \hat{X}_{t}, \widehat{\alpha}, \hat{Y}_{t}, \hat{Z}_{t}, \hat{\eta}_{t}\right)\left(\hat{Y}_{t}-Y_{t}\right)+\partial_{\eta} \mathcal{H}\left(t, \hat{X}_{t}, \widehat{\alpha}, \hat{Y}_{t}, \hat{Z}_{t}, \hat{\eta}_{t}\right)\left(\hat{\eta}_{t}-\eta_{t}\right)
\end{aligned}
$$

and the latter term $\partial_{\eta} \mathcal{H}\left(t, \hat{X}_{t}, \widehat{\alpha}, \hat{Y}_{t}, \hat{\eta}_{t}\right)\left(\hat{\eta}_{t}-\eta_{t}\right) \geq 0$ Therefore,

$$
\begin{aligned}
J\left(\hat{\alpha}_{t}, \hat{\eta}_{t}\right)-J\left(\hat{\alpha}_{t}, \eta_{t}\right) \\
\geq \mathbb{E}\left[\int_{0}^{T} \mathcal{H}\left(t, \hat{X}_{t}, \hat{\alpha}_{t}, \hat{Y}_{t}, \hat{Z}_{t}, \hat{\eta}_{t}\right)-\mathcal{H}\left(t, X_{t}, \hat{\alpha}_{t}, Y_{t}, \hat{Z}_{t}, \eta_{t}\right) d t\right. \\
\left.\quad-\int_{0}^{T}\left(d \hat{X}_{t} \hat{Y}_{t}-d X_{t} Y_{t}\right)+\left(\hat{X}_{t}-X_{t}\right) d Y_{t}+\int_{0}^{T}\left(d \hat{X}_{t}-d X_{t}\right) Y_{t}\right] \geq 0
\end{aligned}
$$

and so $\eta_{t}$ achieves a maximum with respect to any admissible control $\alpha_{t}$.

A similar argument yields that, again by convexity of $\mathrm{g}$ and Ito's formula, comparing the optimal strategy $\hat{\alpha}_{t}$ with an arbitrary control $\alpha_{t}$, we get $J\left(\hat{\alpha}_{t}, \hat{\eta}_{t}\right) \leq J\left(\alpha_{t}, \hat{\eta}_{t}\right)$. The proof is provided for completeness. Under the assumption of convexity, we have that

$$
g\left(\hat{X}_{T}\right)-g\left(X_{T}\right) \leq\left(\hat{X}_{T}-X_{T}\right) \hat{Y}_{T}=\int_{0}^{T}\left(\hat{X}_{t}-X_{t}\right) d \hat{Y}_{t}+\int_{0}^{T}\left(d \hat{X}_{t}-d X_{t}\right) \hat{Y}_{t}
$$

Furthermore, we have

$$
\begin{aligned}
& \mathbb{E}\left[\int_{0}^{T} f\left(t, \mu_{t}, \hat{X}_{t}, \hat{\alpha}_{t}, \widehat{\eta}_{t}\right)-f\left(t, \mu_{t}, X_{t}, \alpha_{t}, \widehat{\eta}_{t}\right) d t\right] \\
& =\mathbb{E}\left[\int_{0}^{T} \mathcal{H}\left(t, \hat{X}_{t}, \hat{\alpha}_{t}, \hat{Y}_{t}, \hat{Z}_{t}, \widehat{\eta}\right)-\mathcal{H}\left(t, X_{t}, \alpha_{t}, \hat{Y}_{t}, \hat{Z}_{t}, \widehat{\eta}\right) d t-\int_{0}^{T}\left(d \hat{X}_{t}-d X_{t}\right) \hat{Y}_{t}\right]
\end{aligned}
$$

where the second statement is $\leq 0$, so again, adding up the bounds for $\mathrm{f}$ and $\mathrm{g}$ yields

$$
\begin{aligned}
& J\left(\hat{\alpha}_{t}, \hat{\eta}_{t}\right)-J\left(\alpha_{t}, \hat{\eta}_{t}\right) \\
& \quad \leq \mathbb{E}\left[\int_{0}^{T} \mathcal{H}\left(t, \hat{X}_{t}, \hat{\alpha}_{t}, \hat{Y}_{t}, \hat{Z}_{t}, \widehat{\eta}\right)-\mathcal{H}\left(t, X_{t}, \alpha_{t}, \hat{Y}_{t}, \hat{Z}_{t}, \widehat{\eta}\right) d t+\int_{0}^{t}\left(\hat{X}_{t}-X_{t}\right) d \hat{Y}_{t}\right] \\
& \quad \leq \mathbb{E}\left[\int_{0}^{T} \mathcal{H}\left(t, \hat{X}_{t}, \hat{\alpha}_{t}, \hat{Y}_{t}, \hat{Z}_{t}, \widehat{\eta}\right)-\mathcal{H}\left(t, X_{t}, \alpha_{t}, \hat{Y}_{t}, \hat{Z}_{t}, \hat{\eta}\right) d t-\int_{0}^{t}\left(\hat{X}_{t}-X_{t}\right) \partial_{x} \mathcal{H}\left(t, \hat{X}_{t}, \hat{\alpha}_{t}, \hat{Y}_{t}, \hat{Z}_{t} \cdot \hat{\eta}\right)\right] \leq 0
\end{aligned}
$$

by convexity of $\mathcal{H}$ with respect to $(x, \alpha)$ 
Lemma 4.2.1. Under the same assumptions of Lemma (4.1.1), we have that

$$
\begin{aligned}
J\left(\widehat{\alpha}^{\mu}, \widehat{\eta}^{\mu} ; \mu\right) & +\left\langle x_{0}^{\prime}-x_{0}, Y_{0}\right\rangle+\lambda \mathbb{E}\left[\int_{0}^{T}\left|\widehat{\alpha}^{\mu^{\prime}}-\alpha_{t}\right|^{2} d t\right]-C \mathbb{E}\left[\int_{0}^{T}\left|\widehat{\eta}_{t}^{\mu}-\widehat{\eta}_{t}^{\mu^{\prime}}\right|^{2} d t\right] \\
& \leq J\left(\left[\widehat{\alpha}^{\mu^{\prime}}, \widehat{\eta}^{\mu^{\prime}}, \mu^{\prime}\right] ; \mu\right)+\mathbb{E}\left[\int_{0}^{T}\left\langle b_{0}\left(t, \mu^{\prime}\right)-b_{0}(t, \mu), Y_{t}\right\rangle d t\right]
\end{aligned}
$$

where $J\left(\left[\widehat{\alpha}^{\mu^{\prime}}, \widehat{\eta}^{\mu^{\prime}}, \mu^{\prime}\right] ; \mu\right)$ uses the optimal controls $\widehat{\alpha}, \widehat{\eta}$ with respect to the measure $\mu^{\prime}$, but evaluates the cost function with respect to the measure $\mu$.

Furthermore, Assumptions 4.1.4 and 4.1.5 give us

$$
\begin{aligned}
J\left(\widehat{\alpha}^{\mu}, \widehat{\eta}^{\mu} ; \mu\right) & +\left\langle x_{0}^{\prime}-x_{0}, Y_{0}^{\prime}\right\rangle-\lambda_{\eta} \mathbb{E}\left[\int_{0}^{T}\left|\widehat{\eta}_{t}^{\mu}-\widehat{\eta}_{t}^{\mu^{\prime}}\right|^{2} d t\right] \\
& \geq J\left(\left[\widehat{\alpha}^{\mu^{\prime}}, \widehat{\eta}^{\mu^{\prime}}, \mu^{\prime}\right] ; \mu\right)+\mathbb{E}\left[\int_{0}^{T}\left\langle b_{0}\left(t, \mu^{\prime}\right)-b_{0}(t, \mu), Y_{t}^{\prime}\right\rangle d t\right]
\end{aligned}
$$

where $Y_{t}^{\prime}$ in the second expression is the adjoint process based on the BSDE driven by $\left(X_{t}^{\prime}, \widehat{\eta}_{t}^{\mu^{\prime}}\right)$

Proof. We substitute in Assumptions (4.1.2),(4.1.4) in the respective expressions in the proof of Theorem (4.2.1).

In particular, we have that

$$
\left\langle X_{T}^{\prime}-X_{T}, Y_{T}\right\rangle=\left\langle X_{0}^{\prime}-X_{0}, Y_{0}\right\rangle+\int_{0}^{T} d\left\langle X_{t}^{\prime}-X_{t}, Y_{t}\right\rangle
$$

and the contribution of the drift terms with respect to the measures $\mu, \mu^{\prime}$ comes from the equation

$$
\left\langle b\left(t, x, \mu^{\prime}, \alpha, \eta\right), y\right\rangle+f(t, x, \mu, \alpha, \eta)=\mathcal{H}(t, x, \mu, \alpha, y, \eta)+\left\langle b_{0}\left(t, \mu^{\prime}\right)-b_{0}(t, \mu), y\right\rangle
$$

Corollary 4.2.1. There exists constant $C, C^{\prime} \in \mathbb{R}^{+}$such that

$$
\begin{aligned}
J\left(\widehat{\alpha}^{\mu}, \widehat{\eta}^{\mu} ; \mu\right) & +C\left\langle x_{0}^{\prime}-x_{0}, Y_{0}-Y_{0}^{\prime}\right\rangle+\lambda \mathbb{E}\left[\int_{0}^{T}\left|\widehat{\alpha}^{\mu^{\prime}}-\alpha_{t}\right|^{2} d t\right] \\
& \leq J\left(\left[\widehat{\alpha}^{\mu^{\prime}}, \widehat{\eta}^{\mu^{\prime}}, \mu^{\prime}\right] ; \mu\right)+C^{\prime} \mathbb{E}\left[\int_{0}^{T}\left\langle b_{0}\left(t, \mu^{\prime}\right)-b_{0}(t, \mu), Y_{t}-Y_{t}^{\prime}\right\rangle d t\right]
\end{aligned}
$$




\subsection{Mean-Field FBSDE}

The optimal controls $\widehat{\alpha}, \widehat{\eta}$ can be inserted into the stochastic differential equation for the state $X_{t}$, as well as a stochastic differential equation for the adjoint process $Y_{t}$. The dependency of the feedback form for $\widehat{\alpha}, \widehat{\eta}$ on $\left(X_{t}, Y_{t}\right)$ creates a coupling between these SDEs and allows us to derive a McKean-Vlasov type forwards backwards stochastic differential equation (FBSDE).

The FBSDE can be written as

$$
\begin{aligned}
d X_{t} & =b\left(t, X_{t}, \mu_{t}, \widehat{\alpha}_{t}, \widehat{\eta}_{t}\right) d t+\sigma d W_{t} \\
d Y_{t} & =-\partial_{x} \mathcal{H}\left(t, X_{t}, \mu_{t}, \widehat{\alpha}_{t}, Y_{t}, Z_{t}, \widehat{\eta}_{t}\right)+Z_{t} d W_{t}
\end{aligned}
$$

with initial condition $X_{0}=x_{0}$ and terminal condition $Y_{T}=\partial_{x} g\left(X_{t}, \mu_{t}\right)$.

The existence and uniqueness of an equilibrium measure $\mu_{t}$ for the mean-field game comes as a result of the Lipschitz continuity of the parameters in the problem, as well as Lipschitz regularity for the measure $\mu_{t}$ with respect to the 2-Wasserstein distance.

Theorem 4.3.1. Under the assumptions (4.1.1,4.1.2,4.1.4.4.1.5,4.1.6,4.1.7), (4.25) has a solution.

Lemma 4.3.1. Given a measure flow $\mu_{t} \in \mathcal{P}_{2}\left(\mathbb{R}^{d}\right)$, the FBSDE has a unique solution and there exists a constant $c$, and a function $u(t, x ; \mu)$ such that

$$
\left|u(t, x ; \mu)-u\left(t, x^{\prime} ; \mu\right)\right| \leq c\left|x-x^{\prime}\right|
$$

and $\mathbb{P}$-almost surely, $Y_{t}=u(t, x ; \mu)$.

Proof. Standard results from (Delarue (2002)) gives us a unique solution of the FBSDE in a local neighbourhood of $t$, due to the Lipschitz structure of the backwards SDE in $(\mathrm{x}, \mathrm{y})$.

We note that $\widehat{\eta}_{t}=\widehat{\eta}\left(t, X_{t}, \mu_{t}, Y_{t}, \alpha_{t}\right)$ in feedback form, so that we can view the FBSDE as the solution of a standard optimization problem.

We now claim that

$$
\left|Y_{t}^{x}-Y_{t}^{x^{\prime}}\right|^{2} \leq c\left|x-x^{\prime}\right|^{2}, \quad \forall x, x^{\prime} \in \mathbb{R}^{d}
$$

so that Delarue (2002) implies that a unique solution exists on $[0, \mathrm{~T}]$

By the estimates in (Lemma 4.2.1), we can deduce that there exists some constant $c$ 
independent of $t$ such that

$$
\mathbb{E}\left[\int_{t}^{T}\left|\widehat{\eta}_{t}^{t_{0}, x_{0}}-\widehat{\eta}_{t}^{t_{0}, x_{0}^{\prime}}\right|^{2}\right] d t+\mathbb{E}\left[\int_{t}^{T}\left|\widehat{\alpha}_{t}^{t_{0}, x_{0}}-\widehat{\alpha}_{t}^{t_{0}, x_{0}^{\prime}}\right|^{2}\right] d t \leq c\left\langle x_{0}^{\prime}-x_{0}, Y_{t_{0}}^{t_{0}, x_{0}^{\prime}}-Y_{t_{0}}^{t_{0}, x_{0}}\right\rangle
$$

By standard SDE (see Karatzas and Shreve (2014)) and BSDE (see Karoui and Mazliak (1997), Yong and Zhou (1999)) estimates, we have, for some constant $C$

$$
\begin{aligned}
\mathbb{E}\left[\sup _{t_{0} \leq t \leq T}\left|X_{t}^{t_{0}, x_{0}}-X_{t}^{t_{0}, x_{0}^{\prime}}\right|^{2}\right]+\mathbb{E}\left[\sup _{t_{0} \leq t \leq T}\left|Y_{t}^{t_{0}, x_{0}}-Y_{t}^{t_{0}, x_{0}^{\prime}}\right|^{2}\right] \\
\leq C\left(\mathbb{E}\left[\int_{t_{0}}^{T}\left|\widehat{\alpha}_{t}^{t_{0}, x_{0}}-\widehat{\alpha}_{t}^{t_{0}, x_{0}^{\prime}}\right|^{2}\right]+\mathbb{E}\left[\int_{t_{0}}^{T}\left|\widehat{\eta}_{t}^{t_{0}, x_{0}}-\widehat{\eta}_{t}^{t_{0}, x_{0}^{\prime}}\right|^{2}\right]\right)
\end{aligned}
$$

and so combining the estimates, we obtain

$$
\mathbb{E}\left[\sup _{t_{0} \leq t \leq T}\left|X_{t}^{t_{0}, x_{0}}-X_{t}^{t_{0}, x_{0}^{\prime}}\right|^{2}\right]+\mathbb{E}\left[\sup _{t_{0} \leq t \leq T}\left|Y_{t}^{t_{0}, x_{0}}-Y_{t}^{t_{0}, x_{0}^{\prime}}\right|^{2}\right] \leq c^{\prime}\left(\left\langle x_{0}^{\prime}-x_{0}, Y_{t_{0}}^{t_{0}, x_{0}^{\prime}}-Y_{t_{0}}^{t_{0}, x_{0}}\right\rangle\right)
$$

for some constant $c^{\prime}$, which proves (4.27)

The representation $u(t, x)=Y^{t, x}$ almost surely comes from Lipschitz continuity in $x$ and Delarue (2002).

Definition 4.3.1. $\Phi: \mathcal{P}\left(\mathbb{R}^{d}\right) \rightarrow \mathcal{P}\left(\mathbb{R}^{d}\right)$ is a mapping of the measure $\mu_{t}$ to Law $\left(X_{t}\right)$ under the dynamics induced by $(\widehat{\alpha}, \widehat{\eta})$.

$\Phi$ is the mapping of the frozen measure flow to the distribution of $X_{t}$. In order for a mean-field game to exist, we need to show that there exists a measure flow $\mu_{t}$ such that the consistency equation $\Phi\left(\mu_{t}\right)=\mu_{t}$ is satisfied.

Lemma 4.3.2. The system (4.25) is solvable if we assume that $\left|\partial_{x} g\right|,\left|\partial_{x} f\right| \leq c$ for some constant c, as well Assumptions (4.1.1,4.1.2,4.1.4,4.1.5,4.1.6,4.1.7).

\section{Proof. First Step}

We first establish a bound on the derivatives of the Hamiltonian. In particular,

$$
\left|\partial_{x} H\left(t, x, \mu_{t}, \widehat{\alpha}_{t}, y, \widehat{\eta}_{t}\right)\right| \leq C_{1}+C_{2}|y|
$$

and so, by Gronwall's Lemma, we observe that there is a constant $c$ depending on $C_{1}, C_{2}$ such that

$$
\left|Y_{t}^{x_{0}}\right| \leq c, \quad \forall 0 \leq t \leq T
$$


and therefore there is a (possibly different) constant $c$ such that

$$
\left|\widehat{\alpha}\left(t, X_{t}, \mu_{t}, Y_{t}\right)\right|,\left|\widehat{\eta}\left(t, X_{t}, \mu_{t}, Y_{t}\right)\right| \leq c, \quad \forall 0 \leq t \leq T
$$

Finally these estimates tell us that there is a constant $C$ such that

$$
\mathbb{E}\left[\sup _{0 \leq t \leq T}\left|X_{t}^{x_{0}, \mu}\right|^{4}\right]<C
$$

We therefore restrict ourselves to the set of measures with finite fourth moments

$$
\mathcal{E}=\left\{\mu \in \mathcal{P}_{4} \mid M_{4}(\mu) \leq C\right\}
$$

\section{Second Step}

We can see that $\Phi$ is relatively compact since

$$
\left|X_{t}^{x_{0} ; \mu}-X_{s}^{x_{0} ; \mu}\right| \leq c\left[(t-s)\left(1+\sup _{0 \leq r \leq T}\left|X_{r}^{x_{0} ; \mu}\right|\right)+\left|B_{t}-B_{s}\right|\right]
$$

\section{Third Step}

We first note that

$$
W_{1}\left(\Phi(\mu), \Phi\left(\mu^{\prime}\right)\right) \leq \mathbb{E}\left[\sup _{0 \leq t \leq T} \mid X_{t}^{x_{0} ; \mu}-X_{t}^{x_{0} ; \mu^{\prime}}\right]
$$

and from the same proof as in Lemma 4.2.1, we obtain

$$
\begin{aligned}
J(\widehat{\alpha}, \widehat{\eta} ; \mu) & +\lambda \mathbb{E}\left[\int_{0}^{T}\left|\widehat{\alpha}_{t}^{\prime}-\widehat{\alpha}_{t}\right|^{2} d t\right] \\
& \leq J\left(\left[\widehat{\alpha}^{\prime}, \widehat{\eta}^{\prime}, \mu^{\prime}\right] ; \mu\right)+\mathbb{E}\left[\int_{0}^{T}\left\langle b_{0}\left(t, \mu_{t}^{\prime}\right)-b_{0}\left(t, \mu_{t}\right), Y_{t}\right\rangle d t\right]
\end{aligned}
$$

so that

$$
\begin{aligned}
\lambda \mathbb{E}\left[\int_{0}^{T}\left|\widehat{\alpha}_{t}^{\prime}-\widehat{\alpha}_{t}\right|^{2} d t\right] \leq & J\left(\widehat{\alpha}, \widehat{\eta} ; \mu^{\prime}\right)-J(\widehat{\alpha}, \widehat{\eta} ; \mu)+J\left(\left[\widehat{\alpha}^{\prime}, \widehat{\eta}^{\prime}, \mu^{\prime}\right] ; \mu\right)-J\left(\widehat{\alpha}^{\prime}, \widehat{\eta}^{\prime} ; \mu^{\prime}\right) \\
& +C \mathbb{E}\left[\int_{0}^{T}\left\langle b_{0}\left(t, \mu_{t}^{\prime}\right)-b_{0}\left(t, \mu_{t}\right), Y_{t}-Y_{t}^{\prime}\right\rangle d t\right]
\end{aligned}
$$

We define the controlled diffusion

$$
d U_{t}=\left[b_{0}\left(t, \mu_{t}^{\prime}\right)+b_{1}(t) U_{t}+b_{2}(t) \widehat{\alpha}_{t}+b_{3}(t) \widehat{\eta}_{t}\right] d t+\sigma d W_{t}, \quad U_{0}=x_{0}
$$

from which Gronwall's Lemma implies that there is some constant $C$ (which may differ 
line by line) such that

$$
\mathbb{E}\left[\sup _{0 \leq t \leq T}\left|X_{t}^{x_{0} ; \mu}-U_{t}\right|^{2}\right] \leq C \int_{0}^{T} W_{2}^{2}\left(\mu_{t}, \mu_{t}^{\prime}\right) d t
$$

By Assumption 4.1.5

$$
J\left(\widehat{\alpha}, \widehat{\eta} ; \mu^{\prime}\right)-J(\widehat{\alpha}, \widehat{\eta}, \mu)+J\left(\left[\widehat{\alpha}^{\prime}, \widehat{\eta}^{\prime} ; \mu^{\prime}\right] ; \mu\right)-J\left(\widehat{\alpha}^{\prime}, \widehat{\eta}^{\prime} ; \mu^{\prime}\right) \leq C\left(\int_{0}^{T} W_{2}^{2}\left(\mu_{t}, \mu_{t}^{\prime}\right)\right)^{1 / 2}
$$

which allows us to obtain

$$
\int_{0}^{T}\left|\widehat{\alpha}^{\prime}-\widehat{\alpha}\right|^{2} d t \leq C \int_{0}^{T} W_{2}^{2}\left(\mu_{t}, \mu_{t}^{\prime}\right) d t
$$

We can use the approximation for $\eta$ from Lemma 4.2.1 with Assumption 4.1.5 and derive a similar result for $\int_{0}^{T}\left|\widehat{\eta}^{\prime}-\widehat{\eta}\right|^{2} d t$ by using (4.43) which, when combining the above result with Assumption 4.1.2, (4.41) and (4.43), gives us

$\mathbb{E}\left[\int_{0}^{T}\left|\widehat{\alpha}_{t}^{\prime}-\widehat{\alpha}_{t}\right|^{2} d t\right]+\mathbb{E}\left[\int_{0}^{T}\left|\widehat{\eta}_{t}^{\prime}-\widehat{\eta}_{t}\right|^{2} d t\right]+\mathbb{E}\left[\sup _{0 \leq t \leq T}\left|X_{t}^{x_{0} ; \mu}-X_{t}^{x_{0} ; \mu^{\prime}}\right|^{2}\right] \leq C\left(\int_{0}^{T} W_{2}^{2}\left(\mu_{t}, \mu_{t}^{\prime}\right)\right)^{1 / 2}$

by Gronwall's Lemma. Holder's inequality for probability spaces, in conjunction with the fact that $\mu \in \mathcal{E}$ has bounded $4^{\text {th }}$ order moments, then gives

$$
W_{1}\left(\Phi(\mu), \Phi\left(\mu^{\prime}\right)\right) \leq c\left(\int_{0}^{T} W_{2}^{2}\left(\mu_{t}, \mu_{t}^{\prime}\right)\right)^{1 / 4} \leq c\left(\int_{0}^{T} W_{1}^{1 / 2}\left(\mu_{t}, \mu_{t}^{\prime}\right)\right)^{1 / 4}
$$

from which we can conclude that $\Phi$ is continuous on $\mathcal{E}$ with respect to the 1-Wasserstein distance $W_{1}$.

Therefore, by Schauder's fixed point theorem, there exists an equilibrium measure $\mu_{t}$ for the mean-field game and so (4.25) is solvable.

\section{$4.4 \quad \epsilon$-Nash equilibrium}

Assumption 4.4.1. The cost functional $f(t, x, \mu, \alpha, \eta)$ satisfies

$$
\int_{0}^{T}\left|\beta_{t}\right|^{2} d t \rightarrow \infty \Longrightarrow \int_{0}^{T} f\left(t, x, \mu, \beta_{t}, \widehat{\eta}_{t}\right) d t \rightarrow \infty
$$

where $\widehat{\eta}=\widehat{\eta}\left(t, X_{t}, \beta_{t}, Y_{t}\right)$ in feedback form. 
A consequence of this assumption is that a player's control must be almost surely bounded. The following result is provided as reference during the proof.

Lemma 4.4.1. [Theorem 10.2.1 Rachev and Rüschendorf (1998)] Given $\mu \in \mathcal{P}_{d+5}\left(\mathbb{R}^{d}\right)$, we have that

$$
\mathbb{E}\left[W_{2}^{2}\left(\bar{\mu}^{N}, \mu\right)\right] \leq C N^{-2 /(d+4)}
$$

where $C$ is a constant that depends on $d$ and $M_{d+5}(\mu)$, and $\bar{\mu}^{N}$ denotes the empirical measure for a sample of size $N$ with respect to the probability measure $\mu$

Before we proceed, there are a few definitions which will be used in order to (hopefully) avoid confusion when differentiating the finite player game from the mean-field game.

Definition 4.4.1. We let $\bar{J}^{N, i}(\boldsymbol{\alpha})$ denote the cost function of the $i^{\text {th }}$ player in an $N$ player game, when the players use the strategy $\boldsymbol{\alpha}$. In contrast, $\bar{J}(\boldsymbol{\alpha})$ will denote the cost function of the $i^{\text {th }}$ player in the mean-field game when the players use the strategy $\boldsymbol{\alpha}$. Similarly, $\widehat{\alpha}_{t}^{N, i}$ is used when the $i^{\text {th }}$ player employs the mean-field optimal strategy in the $N$-player game.

$\mu_{t}^{N}$ is the empirical distribution in the $N$-player version of the game.

$\bar{X}_{t}^{i}$ is used to denote the $i^{\text {th }}$ player's state in the mean-field game, while $X_{t}^{i}$ denotes the state in the $N$-player game.

Theorem 4.4.1. Under Assumptions (4.1.1,4.1.2,4.1.4,4.1.5,4.1.6,4.1.7,4.4.1), the strategies $\alpha_{t}^{N, i}$ form an $\epsilon$-Nash equilibrium of the N-player game. Specifically, we have

- $\epsilon_{N} \leq c N^{-1 /(d+4)}$

- $\bar{J}^{N, i}\left(\beta^{i} ; \boldsymbol{\alpha}^{-i}\right) \geq \bar{J}^{N, i}(\boldsymbol{\alpha})-\epsilon_{N}$

for some constant $c$ and sequence of positive real numbers $\left\{\epsilon_{N}\right\}_{N \in \mathbb{N}}$ where $\beta^{i} \in \mathcal{A}$ and $\lim _{N \rightarrow \infty} \epsilon_{N}=0$

Proof. By exchangeability, the problem is symmetric for any particular player and so we can assume without loss of generality that $i=1$.

We first define the process $U_{t}^{1}$ to be the dynamics of the player under an arbitrary admissible control $\beta_{t}$.

$$
d U_{t}^{1}=b\left(t, U_{t}^{1}, \mu_{t}, \beta_{t}^{1}, \eta_{t}^{1}\right) d t+\sigma d W_{t}^{1}
$$

By Gronwall's inequality, we have that

$$
\mathbb{E}\left[\sup _{0 \leq t \leq T}\left|U_{t}^{1}\right|^{2}\right] \leq c\left(1+\mathbb{E}\left[\int_{0}^{T}\left|\beta_{t}^{1}\right|^{2}+\left|\eta_{t}^{1}\right|^{2} d t\right]\right)
$$


and, observing that $\mathbb{E}\left[\sup _{0 \leq t \leq T}\left|U_{t}^{1}\right|^{2}\right]<c$ we get that

$$
\frac{1}{N} \sum_{j=1}^{N} \mathbb{E}\left[\sup _{0 \leq t \leq T}\left|U_{t}^{1}\right|^{2}\right] \leq c\left(1+\frac{1}{N} \mathbb{E}\left[\int_{0}^{T}\left|\beta_{t}^{1}\right|^{2}+\left|\eta_{t}^{1}\right|^{2} d t\right]\right)
$$

Then, Snitzman's proof allows us to derive a uniform bound of

$$
\max _{1 \leq i \leq N} \mathbb{E}\left[\sup _{0 \leq t \leq T}\left|X_{t}^{i}-\bar{X}_{t}^{i}\right|^{2}\right] \leq c N^{-2 /(d+4)}
$$

so that the inequality

$$
W_{2}^{2}\left(\bar{\mu}_{t}^{N}, \mu_{t}\right) \leq \frac{2}{N} \sum_{i=1}^{N}\left|X_{t}^{i}-\bar{X}_{t}^{i}\right|^{2}+2 W_{2}^{2}\left(\frac{1}{N} \delta_{\bar{X}_{t}^{i}}, \mu_{t}\right)
$$

gives us the estimate

$$
\sup _{0 \leq t \leq T} \mathbb{E}\left[W_{2}^{2}\left(\bar{\mu}_{t}^{N}, \mu_{t}\right)\right] \leq c N^{-2 /(d+4)}
$$

Lipschitz properties of $f, g$ give us the bound of

$$
\begin{aligned}
& \left|J-J^{N}\right| \\
& \quad=\left|\mathbb{E}\left[g\left(X_{T}, \mu_{T}\right)+\int_{0}^{T} f\left(t, \bar{X}_{t}^{i}, \mu_{t}, \widehat{\alpha}_{t}^{i}, \widehat{\eta}_{t}^{i}\right) d t-g\left(X_{T}, \mu_{T}^{N}\right)-\int_{0}^{T} f\left(t, X_{t}^{i}, \mu_{t}^{N}, \widehat{\alpha}_{t}^{N, i}, \widehat{\eta}_{t}^{N, i}\right) d t\right]\right| \\
& \quad \leq c \mathbb{E}\left[\left(1+\left|\bar{X}_{T}^{i}\right|^{2}+\left|X_{T}^{i}\right|^{2}+\frac{1}{N} \sum_{j=1}^{N}\left|X_{j}^{i}\right|^{2}\right)\right]^{1 / 2} \mathbb{E}\left[\left|\bar{X}_{t}^{i}-X_{t}^{i}\right|^{2}+W_{2}^{2}\left(\mu_{t}, \mu_{t}^{N}\right)\right]^{1 / 2} \\
& \quad+c \int_{0}^{T}\left(\mathbb{E}\left[\left(1+\left|\bar{X}_{t}^{i}\right|^{2}+\left|X_{t}^{i}\right|^{2}+\left|\widehat{\alpha}_{t}^{i}\right|^{2}+\left|\widehat{\alpha}_{t}^{N, i}\right|^{2}+\left|\widehat{\eta}_{t}^{i}\right|^{2}+\left|\widehat{\eta}_{t}^{N, i}\right|^{2}+\frac{1}{N} \sum_{j=1}^{N}\left|X_{j}^{i}\right|^{2}\right) d t\right]^{1 / 2}\right. \\
& \left.\quad \times \mathbb{E}\left[\left|\bar{X}_{t}^{i}-X_{t}^{i}\right|^{2}+\left|\widehat{\alpha}_{t}^{i}-\widehat{\alpha}_{t}^{N, i}\right|^{2}+\left|\widehat{\eta}_{t}^{i}-\widehat{\eta}_{t}^{N, i}\right|^{2}+W_{2}^{2}\left(\mu_{t}, \mu_{t}^{N}\right)\right]^{1 / 2}\right) d t
\end{aligned}
$$

so that we can use our previous bounds to obtain

$$
\begin{aligned}
\left|J-J^{N}\right| & \leq c \mathbb{E}\left[\left|\bar{X}_{T}^{i}-X_{T}^{i}\right|^{2}+W_{2}^{2}\left(\mu_{t}, \mu_{t}^{N}\right)\right]^{1 / 2} \\
& +c\left(\int_{0}^{T} \mathbb{E}\left[\left|\bar{X}_{t}^{i}-X_{t}^{i}\right|^{2}+\left|\widehat{\alpha}_{t}^{i}-\widehat{\alpha}_{t}^{N, i}\right|^{2}+\left|\widehat{\eta}_{t}^{i}-\widehat{\eta}_{t}^{N, i}\right|^{2}+W_{2}^{2}\left(\mu_{t}, \mu_{t}^{N}\right)\right]^{1 / 2}\right)
\end{aligned}
$$

and hence, since the controls $\widehat{\alpha}, \widehat{\eta}$ are Lipschitz in $X$

$$
\left|\widehat{\alpha}_{t}^{i}-\bar{\alpha}_{t}^{N, i}\right|,\left|\widehat{\eta}_{t}^{i}-\bar{\eta}_{t}^{N, i}\right| \leq C\left|\bar{X}_{t}^{i}-X_{t}^{i}\right|
$$


we have that

$$
\bar{J}^{N}=J+O\left(N^{-1 /(d+4)}\right)
$$

Furthermore, we get

$$
\begin{aligned}
& \mathbb{E}\left[\sup _{0 \leq s \leq t}\left|U_{t}^{1}-X_{t}^{1}\right|^{2}\right] \leq \frac{c}{N} \int_{0}^{T} \sum_{j=1}^{N} \mathbb{E}\left[\sup _{0 \leq r \leq s}\left|U_{r}^{j}-X_{r}^{j}\right|^{2}\right] d s \\
&+c \mathbb{E} \int_{0}^{T}\left|\beta_{t}^{1}-\alpha_{t}^{N, 1}\right|^{2} d t+c \mathbb{E} \int_{0}^{T}\left|\eta_{t}^{1}-\eta_{t}^{N, 1}\right|^{2} d t \\
& \mathbb{E}\left[\sup _{0 \leq s \leq t}\left|U_{t}^{i}-X_{t}^{i}\right|^{2}\right] \leq \frac{c}{N} \int_{0}^{T} \sum_{j=1}^{N} \mathbb{E}\left[\sup _{0 \leq r \leq s}\left|U_{r}^{j}-X_{r}^{j}\right|^{2}\right] d s
\end{aligned}
$$

so that Gronwall's inequality gives

$$
\frac{1}{N} \sum_{j=1}^{N} \mathbb{E}\left[\sup _{0 \leq t \leq T}\left|U_{t}^{j}-X_{t}^{j}\right|^{2}\right] \leq \frac{c}{N}\left(\mathbb{E} \int_{0}^{T}\left|\beta_{t}^{1}-\alpha_{t}^{N, 1}\right|^{2} d t+\mathbb{E} \int_{0}^{T}\left|\eta_{t}^{1}-\eta_{t}^{N, 1}\right|^{2} d t\right)
$$

and so therefore

$$
\sup _{0 \leq t \leq T} \mathbb{E}\left[\left|U_{t}^{i}-X_{t}^{i}\right|^{2}\right] \leq \frac{c}{N}\left(\mathbb{E} \int_{0}^{T}\left|\beta_{t}^{1}-\alpha_{t}^{N, 1}\right|^{2} d t+\mathbb{E} \int_{0}^{T}\left|\eta_{t}^{1}-\eta_{t}^{N, 1}\right|^{2} d t\right)
$$

If we fix a constant $A>0$, then we can see that there exists a constant $C_{A}$ such that

$$
\mathbb{E}\left[\int_{0}^{T}\left|\beta_{t}^{1}\right|^{2} d t\right] \leq A \Longrightarrow \max _{2 \leq i \leq N} \sup _{0 \leq t \leq T} \mathbb{E}\left[\left|U_{t}^{i}-\bar{X}_{t}^{i}\right|^{2}\right] \leq C_{A} N^{-2 /(d+4)}
$$

from which we can then obtain the bound

$$
\frac{1}{N-1} \sum_{j=2}^{N} \mathbb{E}\left[\left|U_{t}^{j}-\bar{X}_{t}^{j}\right|^{2}\right] \leq C_{A} N^{-2 /(d+4)}
$$

Applying a triangle inequality for the Wasserstein distance, we get

$$
\begin{aligned}
\mathbb{E}\left[W_{2}^{2}\left(\bar{\nu}_{t}^{N}, \mu_{t}\right)\right] \leq & c \mathbb{E}\left[W_{2}^{2}\left(\frac{1}{N} \sum_{j=1}^{N} \delta_{U_{t}^{j}}, \frac{1}{N-1} \sum_{j=2}^{N} \delta_{U_{t}^{j}}\right)\right] \\
& +\frac{1}{N-1} \sum_{j=2}^{N} \mathbb{E}\left[\left|U_{t}^{j}-\bar{X}_{t}^{j}\right|^{2}\right]+\mathbb{E}\left[W_{2}^{2}\left(\frac{1}{N-1} \sum_{j=2}^{N} \delta_{\bar{X}_{t}^{j}}, \mu_{t}\right)\right]
\end{aligned}
$$


combining with the inequality

$$
\mathbb{E}\left[W_{2}^{2}\left(\frac{1}{N} \sum_{j=1}^{N} \delta_{U_{t}^{j}}, \frac{1}{N-1} \sum_{j=2}^{N} \delta_{U_{t}^{j}}\right)\right] \leq \frac{1}{N(N-1)} \sum_{j=2}^{N} \mathbb{E}\left[\left|U_{t}^{1}-U_{t}^{j}\right|^{2}\right]
$$

as well as previous estimates and Lemma 4.4.1, we get

$$
\mathbb{E}\left[W_{2}^{2}\left(\bar{\nu}_{t}^{N}, \mu_{t}\right)\right] \leq C N^{-2 /(d+4)}
$$

Finally, if we define

$$
d \bar{U}_{t}^{1}=b\left(t, \bar{U}_{t}^{1}, \mu_{t}, \beta_{t}^{1}, \widehat{\eta}_{t}^{1}\right) d t+\sigma d W_{t}^{1}
$$

we get

$$
U_{t}^{1}-\bar{U}_{t}^{1}=\int_{0}^{t}\left[b_{0}\left(s, \bar{\nu}_{s}^{N}\right)-b_{0}\left(s, \mu_{s}\right)\right] d s+\int_{0}^{t} b_{1}(s)\left[U_{s}^{1}-\bar{U}_{s}^{1}\right] d s+\int_{0}^{t} b_{3}(s)\left[\widehat{\eta}^{U}-\widehat{\eta}^{\bar{U}}\right]
$$

From (4.56) and the fact that $\widehat{\eta}$ is Lipschitz with respect to $x$, we can see that an application of Gronwall's inequality gives us

$$
\sup _{0 \leq t \leq T} \mathbb{E}\left[\left|U_{t}^{1}-\bar{U}_{t}^{1}\right|^{2}\right] \leq C_{A} N^{-2 /(d+4)}
$$

(where $C_{A}$ may be a different constant than previously defined) and so, applying the same techniques as in the derivation of (4.57), we get that

$$
\bar{J}^{N, 1}\left(\beta^{1} ; \alpha^{-1}\right) \geq J-C_{A} N^{-1 /(d+4)}
$$

As Assumption 4.4.1 always allows us to find such a constant $A>0$, by combining (4.57) with (4.70) we obtain the bound

$$
\bar{J}^{N, i}\left(\beta^{i} ; \boldsymbol{\alpha}^{-i}\right) \geq \bar{J}^{N, i}(\boldsymbol{\alpha})-\epsilon_{N}
$$

for any $\beta^{i} \in \mathcal{A}$.

\subsubsection{Comments on assumptions}

The concavity assumptions in the paper are in line with the multiplier preference setting, as the relative entropy of the measure change in a setting with Brownian motions is quadratic in nature. In particular, (4.8) and Theorem 4.2.1 are both satisfied when the 
cost functional is quadratic in $\alpha$ and $\eta$ subject to certain constraints on the constants in front of the quadratic terms.

Furthermore, although having bounded derivatives $\partial_{x} f, \partial_{x} g$ is fairly restrictive in general, the class of functions which satisfy these assumptions can be used as approximate solutions to other problems. Unfortunately, the convex-concave nature of the problem makes a formal approximation theorem difficult to prove, however we believe that an approximation theorem like the one in Carmona and Delarue (2013) should hold and leave the proof for a future paper.

\subsection{Linear-Quadratic Game}

The setup for an ambiguity averse agent without a mean-field component in the dynamics has been looked at in Bauso et al. (2016). We will look at a vector-valued version of the problem with a mean-field term in the drift, and characterize its solution.

\subsubsection{Problem Formulation}

Let $W_{t}$ be an n-dimensional Brownian motion, and $x_{t} \in \mathbb{R}^{n}, v_{t} \in \mathbb{R}^{k}$. We wish to find an optimal control $v \in L^{2}\left([0, T], \mathbb{R}^{n}\right)$ to minimize

$$
\begin{aligned}
J(v)= & \mathbb{E}\left[\frac{1}{2} \int_{0}^{T}\left(x_{t}^{\top} Q_{t} x_{t}+v_{t}^{\top} R_{t} v_{t}+\left(x_{t}-S_{t} \mathbb{E}\left[x_{t}\right]\right)^{\top} \bar{Q}_{t}\left(x_{t}-S_{t} \mathbb{E}\left[x_{t}\right]\right)-\phi \log \frac{d \mathbb{Q}}{d \mathbb{P}}\right) d t\right] \\
& +\mathbb{E}\left[\frac{1}{2} x_{T}^{\top} Q_{T} x_{T}+\frac{1}{2}\left(x_{T}-S_{T} \mathbb{E}\left[x_{T}\right]\right)^{\top} \bar{Q}_{T}\left(x_{T}-S_{T} \mathbb{E}\left[x_{T}\right]\right)\right]
\end{aligned}
$$

subject to the dynamics

$$
d x_{t}=\left(A_{t} x_{t}+B_{t} v_{t}+\bar{A}_{t} \mathbb{E}\left[x_{t}\right]+\eta_{t}\right) d t+\sigma_{t}\left(d W_{t}+\eta_{t} d t\right), \quad x(0)=x_{0}
$$

where $\eta_{t}$ is the change in drift induced by the measure change $\frac{d \mathbb{Q}}{d \mathbb{P}}$. Due to Girsanov's theorem, we have an explicit representation for the measure change of the form $\log \frac{d \mathbb{Q}}{d \mathbb{P}}=$ $\eta_{t} \sigma_{t}^{-1} d W_{t}+\frac{1}{2} \eta_{t}^{\top} \sigma_{t}^{-1} \eta_{t} d t$ so that the performance criteria can be rewritten as

$$
\begin{aligned}
J(v)= & \mathbb{E}\left[\frac{1}{2} \int_{0}^{T}\left(x_{t}^{\top} Q_{t} x_{t}+v_{t}^{\top} R_{t} v_{t}+\left(x_{t}-S_{t} \mathbb{E}\left[x_{t}\right]\right)^{\top} \bar{Q}_{t}\left(x_{t}-S_{t} \mathbb{E}\left[x_{t}\right]\right)-\frac{1}{2}\left(\eta_{t}\right)^{\top} \Phi_{t}^{-1} \eta_{t}\right) d t\right] \\
& +\mathbb{E}\left[\frac{1}{2} x_{T}^{\top} Q_{T} x_{T}+\frac{1}{2}\left(x_{T}-S_{T} \mathbb{E}\left[x_{T}\right]\right)^{\top} \bar{Q}_{T}\left(x_{T}-S_{T} \mathbb{E}\left[x_{T}\right]\right)\right]
\end{aligned}
$$


Theorem 4.5.1. The above problem is solvable with optimal control $v=-R^{-1} B^{\top} p$ and measure change $\eta_{t}=\Phi_{t} p$ where $(y, p)$ satisfy the forwards-backwards stochastic differential equation

$$
\begin{aligned}
d y_{t} & =A_{t} y_{t}-\left(B_{t} R^{-1} B_{t}^{\top}-\Phi_{t}\right) p_{t} d t+\bar{A}_{t} \mathbb{E}\left[y_{t}\right] d t+\sigma_{t} d W_{t} \\
-d p_{t} & =\left[\left(Q_{t}+\bar{Q}_{t}\right) y_{t}+A_{t}^{\top} p_{t}\right] d t+\left[-\bar{Q}_{t} S_{t} \mathbb{E}\left[y_{t}\right]-S_{t}^{\top} \bar{Q}_{t}\left(I-S_{t}\right) \mathbb{E}\left[y_{t}\right]+\bar{A}_{t}^{\top} \mathbb{E}\left[p_{t}\right]\right] d t+q_{t} d W_{t} \\
y_{0} & =x_{0} \\
p_{T} & =\left(Q_{t}+\bar{Q}_{t}\right) y_{T}-\bar{Q}_{T} S_{T} \mathbb{E}\left[y_{T}\right]-S_{T}^{\top} \bar{Q}_{T}\left(I-S_{T}\right) \mathbb{E}\left[y_{T}\right]
\end{aligned}
$$

Proof. First, we define

$$
\begin{aligned}
\mathcal{H}(t, x, v, p, \eta)= & \left(A_{t} x_{t}+B_{t} v_{t}+\bar{A}_{t} \mathbb{E}\left[x_{t}\right]+\eta_{t}\right) p_{t} \\
& +\left(x_{t}^{\top} Q_{t} x_{t}+v_{t}^{\top} R_{t} v_{t}+\left(x_{t}-S_{t} \mathbb{E}\left[x_{t}\right]\right)^{\top} \bar{Q}_{t}\left(x_{t}-S_{t} \mathbb{E}\left[x_{t}\right]\right)-\frac{1}{2}\left(\eta_{t}\right)^{\top} \Phi_{t}^{-1} \eta_{t}\right)
\end{aligned}
$$

Then by solving the equations

$$
\begin{aligned}
& \partial_{v} \mathcal{H}(t, x, v, p, \eta)=0 \\
& \partial_{\eta} \mathcal{H}(t, x, v, p, \eta)=0
\end{aligned}
$$

we obtain the optimal controls

$$
\begin{aligned}
v & =-R^{-1} B^{\top} p \\
\eta_{t} & =\Phi_{t} p
\end{aligned}
$$

plugging these back in, and noting that it should satisfy the FBSDE

$$
\begin{aligned}
d X_{t} & =\partial_{y} \mathcal{H}(t, x, v, p, \eta) d t+\sigma_{t} d W_{t} \\
d Y_{t} & =-\partial_{x} \mathcal{H}(t, x, v, p, \eta) d t+q_{t} d W_{t} \\
X_{0} & =x_{0} \\
Y_{T} & =\left(Q_{t}+\bar{Q}_{t}\right) X_{T}-\bar{Q}_{T} S_{T} \mathbb{E}\left[X_{T}\right]-S_{T}^{\top} \bar{Q}_{T}\left(I-S_{T}\right) \mathbb{E}\left[X_{T}\right]
\end{aligned}
$$


we obtain the equations

$$
\begin{aligned}
d y_{t} & =A_{t} y_{t}-\left(B_{t} R^{-1} B_{t}^{\top}-\Phi_{t}\right) p_{t} d t+\bar{A}_{t} \mathbb{E}\left[y_{t}\right] d t+\sigma_{t} d W_{t} \\
-d p_{t} & =\left[\left(Q_{t}+\bar{Q}_{t}\right) y_{t}+A_{t}^{\top} p_{t}\right] d t+\left[-\bar{Q}_{t} S_{t} \mathbb{E}\left[y_{t}\right]-S_{t}^{\top} \bar{Q}_{t}\left(I-S_{t}\right) \mathbb{E}\left[y_{t}\right]+\bar{A}_{t}^{\top} \mathbb{E}\left[p_{t}\right]\right] d t+q_{t} d W_{t} \\
y_{0} & =x_{0} \\
p_{T} & =\left(Q_{t}+\bar{Q}_{t}\right) y_{T}-\bar{Q}_{T} S_{T} \mathbb{E}\left[y_{T}\right]-S_{T}^{\top} \bar{Q}_{T}\left(I-S_{T}\right) \mathbb{E}\left[y_{T}\right]
\end{aligned}
$$

Although the ambiguity averse setting is in general distinct from the standard linearquadratic setup, if $B_{t},\left(R^{-1}-B_{t}^{-1} \Phi\left(B_{t}^{\top}\right)^{-1}\right)$ are invertible for all $t \in[0, T]$, then there is an equivalent linear-quadratic game without ambiguity aversion with the same control, namely obtained by re-parameterizing $R$ to $\left(R^{-1}-B_{t}^{-1} \Phi\left(B_{t}^{\top}\right)^{-1}\right)^{-1}$.

\subsection{2 $\epsilon$-Nash equilibrium}

Since the dynamics under the optimal control of the linear-quadratic mean-field game with ambiguity aversion can be associated with a version of the linear-quadratic meanfield game without ambiguity aversion, the $\epsilon$-Nash equilibrium holds with the same arguments as in Bensoussan et al. (2016). We note that the existence, uniqueness and validity of the $\epsilon$-Nash equilibrium is contingent on Assumption 4.1.2, so that the condition provides a sufficient bound for the disturbance attenuation parameter $\Phi$.

\subsubsection{Comments}

We can see that in the linear-quadratic case, ambiguity aversion is equivalent to reducing the cost of the control in the cost function. Furthermore, Assumption 4.1.2 and the concavity assumption on the Hamiltonian are equivalent in this scenario. We note that when 4.1.2 becomes close to being violated, the optimal control approaches $\infty$, so that it is unattainable. Therefore, for linear-quadratic games, the condition is both necessary and sufficient for the mean-field game to exist.

Bauso et al. (2016) provide a framework for robust mean-field games in which the solution requires iteratively solving two PDE. The setup with Pontryagin maximum principle allows us to use numerical FBSDE schemes, which is an active area of research. Some examples of FBSDE numerical schemes are presented in Douglas et al. (1996), Bender et al. (2008) and Zhao et al. (2014). 


\section{Chapter 5}

\section{Background}

This chapter gives the mathematical background and tools used throughout the thesis.

\subsection{Probability}

A probability space is defined as a triplet $(\Omega, \mathcal{F}, \mu)$ where $\mathcal{F}$ is a $\sigma$-algebra for the space $\Omega$, and $\mu$ is a probability measure defined on $\Omega$.

\subsection{Hamilton-Jacobi-Bellman Principle}

One of the primary techniques used to solve a MFG problem is the Hamilton-JacobiBellman (HJB) equation. We consider a control system

$$
\left\{\begin{array}{l}
\dot{x}(t)=b(t, x(t), u(t)) d t+\sigma(t) d W_{t}+\int_{-\infty}^{\infty} f\left(t, x_{t^{-}}, u_{t^{-}}, y\right) \mu(d y, d t) \\
x(0)=x_{0}
\end{array}\right.
$$

where $x(t)$ is a stochastic process, and $u(t)$ denotes the control.

An agent wishes to minimize her cost function

$$
J(u(\cdot))=\int_{0}^{t} C(t, x(t), u(t)) d t+D(x(T))
$$

Given the constraint $u \in \mathcal{U}$ where

$$
\mathcal{U}=\{u \mid \mathrm{u} \text { is an admissible control }\}
$$


the optimization problem becomes

$$
V^{*}(x, t)=\max _{u \in \mathcal{U}}\left\{\int_{0}^{t} C(x(t), u(t)) d t+D(x(T), u(T))\right\}
$$

The infinitesimal generator for a function $g(t, x, u, y)$ of this process is

$$
\mathcal{L}^{u}=b(t, x, u) \partial_{x} g+\frac{1}{2} \sigma^{2}(t) \partial_{x x} g+\lambda_{x} \int_{-\infty}^{\infty}(g(t, x+f)-g(t, x)) F_{x}(y) d y
$$

\subsection{Pontryagin Stochastic Minimum Principle}

An alternative way to solve stochastic optimal control problems is Pontragin's Stochastic Minimum Principle.

Considering the same stochastic process $X_{t}$ and cost function $J(u(\cdot))$ as before, we can write the Hamiltonian as

$$
H(t, x, u, y)=b(t, x, u) y+f(t, x, u)
$$

and by the envelope theorem, we can write an FBSDE that $(\mathrm{X}, \mathrm{Y})$ must satisfy

$$
\begin{aligned}
d X_{t} & =H_{y}(t, x, y, u) d t+\sigma(t) d W_{t} \\
d Y_{t} & =-H_{x}(t, x, y, u) d t+Z_{t} d W_{t}, \quad Y_{T}=\partial_{x} g\left(X_{T}\right)
\end{aligned}
$$

\subsection{Ansatz}

An important idea in obtaining closed form or semi-closed form solutions in this thesis is the application of an ansatz. Ansatz are an educated guess to the form of the solution, which will generally reduce the complexity of the problem under consideration. (ie. reducing a partial differential equation to a set of coupled ordinary differential equations).

\subsection{Almgren-Criss Framework}

In the Almgren-Criss framework, the agent wishes to liquidate $Q_{0}$ stocks by time $\mathrm{T}$ by sending market orders while incurring a penalty based on the inventory they hold at any moment in time. Let $W_{t}$ be a standard Brownian motion, $\sigma, a, b$ be constants, and $\nu_{t}$ be 
the rate of trading for the agent. The change in inventory of the agent at time $t$ is

$$
d Q_{t}=\nu_{t} d t
$$

The midprice process $S_{t}$ satisfies

$$
d S_{t}=b \nu_{t} d t+\sigma d W_{t}
$$

where $b$ is considered the permanent impact of trading, and $\sigma$ is the volatility of the stock price. Although most models, such as the Black-Scholes framework, assumes a geometric Brownian motion framework, the execution strategies which we consider are generally executed on the order of hours, so an arithmetic Brownian motion is a good approximation across the time frame that we will be working with.

The amount of cash they receive is

$$
d X=\nu_{t}\left(S_{t}-a \nu_{t}\right) d t
$$

where $a$ is the temporary impact of trading. The permanent impact of trading impacts the price of the asset over a long period of time, while the temporary trading impact $a$ only impacts the current execution order. Generally $a$ is due to a number of factors such as transaction costs or crossing the bid-ask spread. This type of limit order book dynamic has been studied and extended in Obizhaeva and Wang (2013), Alfonsi et al. (2008), Alfonsi and Schied (2010) and Alfonsi et al. (2012).

Given a penalty parameter $\phi>0$, the agent wishes to maximize their performance criteria

$$
J(\nu(\cdot))=\mathbb{E}\left[\int_{0}^{T} \nu_{t}\left(S_{t}-a \nu_{t}\right) d t-\alpha Q_{T}^{2}-\phi \int_{0}^{T} Q_{t}^{2} d t\right]
$$

where $\alpha$ is a permanent penalty that is imposed on the agent for shares which were not liquidated.

In this framework, a closed-form solution exists, and the inventory process under the optimal control is

$$
\boldsymbol{Q}_{\boldsymbol{t}}=\boldsymbol{Q}_{\mathbf{0}} \frac{\zeta e^{\gamma(T-t)}-e^{-\gamma(T-t)}}{\zeta e^{\gamma T}-e^{-\gamma T}} \stackrel{\alpha \rightarrow \infty}{\longrightarrow} \boldsymbol{Q}_{\mathbf{0}} \frac{\sinh (\gamma(T-t))}{\sinh (\gamma T)}, \quad \zeta, \gamma \text { are constants }
$$

where

$$
\gamma=\sqrt{\frac{\phi}{k}}, \quad \zeta=\frac{\alpha-\frac{1}{2} b+\sqrt{k \phi}}{\alpha-\frac{1}{2} b-\sqrt{k \phi}}
$$


In this setting, the agent takes into account their aversion to future risk in price movements, as well as the cash amount they receive. However, this framework fails to take into account the response of other agents in the market. In particular, if the direction of the movement for the stock price is known, then traders can and will easily profit off of this information. To account for this, a game-theoretic framework is introduced. In order to account for the presence of the large number of HFTs in the market, a mean-field game approximation is used for tractability.

The temporary penalty with respect to the parameter $\phi$ can be seen as stemming from ambiguity aversion in Cartea et al. (2013), so that the penalty is equivalent to maximizing the following performance criteria

$$
J(\nu(\cdot))=\mathbb{E}\left[\int_{0}^{T} \nu_{t}\left(S_{t}-a \nu_{t}\right) d t-\alpha Q_{T}^{2}-\phi \mathcal{H}(\mathbb{Q} \| \mathbb{P})\right]
$$

where $\mathcal{H}$ is the relative entropy of $\mathrm{Q}$ with respect to $\mathrm{P}$ (or Kullback-Leibler Divergence) 


\section{Chapter 6}

\section{Future work}

Mean-field games and ambiguity aversion are ongoing very active areas of research, with many applications beyond the area of mathematical finance. The effect of ambiguity aversion, in general, is distinct from that of risk aversion, or risk-sensitive games as outlined in Tembine et al. (2011).

There are a number of questions which came up in the course of studying the problems in this thesis which are of future interest.

\subsection{Optimal Execution}

In Chapter 2, a game-theoretic version of the Almgren-Criss model is considered for optimal execution. A verification theorem as well as $\epsilon$-Nash equilibrium is provided, and a semi-closed form solution in the form of coupled Riccatti ODEs is given. The impact of HFTs on the market is shown through simulation studies with parameters which were calibrated to real data.

Although the approximation is reasonable given the number of agents in the market, the most significant assumption in this framework is that of complete information. In reality, traders cannot know the performance criteria $J(\alpha)$ for everyone in the market. A more realistic setting would involve traders whom have only partial information available to them, and attempt to optimize their performance criteria subject to this information. Some research in this direction for mean-field games include Şen and Caines (2014), Caines and Kizilkale (2016), Firoozi and Caines (2016b), Saldi et al. (2017) and Buckdahn et al. (2017). The framework of Şen and Caines (2014) has been applied to the problem in Chapter 2 in a limited manner by Firoozi and Caines (2016a), by assuming that the observed inventory levels of the major and minor agents are noisy.

A different approach to the partial information setting would be through constructing a 
latent alpha process, and using this process to estimate the current direction of the market as in Casgrain and Jaimungal (2017). Estimating in this manner is more consistent with how a trader would view market dynamics, as they would not necessarily assume that the movement in the market is driven by a broker execution strategy. Furthermore, trading based on the latent alpha model could be more robust, as the trader would not need to estimate the numerous parameters which impact the broker's trading strategy.

Another extension would be to model the price formation process of the limit order book through a mean-field approximation of market participants, such as in Gayduk and Nadtochiy (2016). In this framework, the execution strategy of the major agent would affect the beliefs of market participants, and the market participants would place limit and market orders accordingly. This framework would also avoid the need to estimate the broker's parameters, and has the additional benefit of allowing the trader to place limit orders as well, in contrast to the setup in Chapter 2 which implicitly only allows market orders to be placed. The addition of limit orders would also allow the price to be formed endogenously, as the traders would be responsible for placing limit orders, as well as eating through the book via market orders.

\subsection{Systemic Risk}

In Chapter 3, there are two observations which would help capture more realistic dynamics for interbank borrowing and lending.

The first observation is that overnight borrowing is not instantaneous, so a relevant issue for banks would be how borrowing should be performed when it has a delayed effect. The impact of delay in mean-field games was investigated in Carmona et al. (2016), and the effect of ambiguity aversion would be particularly relevant in this context, as the impact of model misspecification is compounded by the delay of any corrective measures taken. The second observation is that banks are not necessarily completely connected, and they should not be equally weighted in the cost functional. A mean-field graphic model as outlined in Guéant (2015) would be more appropriate, as network models form the basis of most of the empirical research in systemic risk, as in Acharya et al. (2017), Billio et al. (2012) and Haldane and May (2011). In this case, the concept of 'congestion' as presented in Guéant (2015) would be analogous to a liquidity crisis in the financial market. Furthermore, this would allow the connections between banks to have heterogeneous weights, so that certain banks may have a sizeable impact on network as a whole. The effect of ambiguity aversion on network models has not been studied to my knowledge. It is also worth mentioning that the default probability results in Chapter 3 were derived 
numerically, but the techniques cannot be applied to mean-field games in general, particularly if there is a common noise process. Given that the model focuses on bank default probabilities and systemic risk, a pertinent question to regulators and risk analysts would be: what can we say about the behavior of the tail distribution for bank log-reserves? A result for static games was derived in Lacker and Ramanan (2017), but there are no such results yet for dynamic games, outside of some very specific instances such as Garnier et al. (2013).

\subsection{Mean-field Games with Ambiguity Aversion}

In Chapter 4, the existence, uniqueness and $\epsilon$-Nash properties of a mean-field game are established for a game with ambiguity averse agents. Although the general result has fairly restrictive assumptions on the structure of the cost function with respect to the state variable $x$, Section 4.5 shows, through an explicit derivation, that these results hold in the linear-quadratic framework as well

The existence and uniqueness theorems in this chapter covers the case of quadratic controls, but has more restrictions when compared to the framework without ambiguity aversion in Carmona and Delarue (2013). The assumptions are clearly necessary and sufficient for mean-field games with quadratic controls, but it is still necessary to establish how certain assumptions (particularly Assumptions 4.1.2, 4.1.4) can be relaxed. The spike variation method has been shown to be very successful for establishing a maximum principle for non-convex cost functions, as seen in Peng (1990),Djehiche et al. (2015),Buckdahn et al. (2016). Given the generality of the cost functions under consideration in these papers, the use of a second order approximation for the Hamiltonian may help generalize the results in Chapter 4.

Anther direction for future research which builds on the results in Chapter 3, and the result in Carmona et al. (2016), would be ambiguity aversion for mean-field games with common noise. Particularly in the case of financial markets, it is unrealistic to assume that agents are subject to idiosyncratic sources of noise. As correlations are an important consideration in financial modeling, a framework incorporating ambiguity aversion with a more general volatility process would potentially allow mean-field games to be applied in many areas of economics and mathematical finance. 


\section{Bibliography}

Acharya, V. V., L. H. Pedersen, T. Philippon, and M. Richardson (2017). Measuring systemic risk. Review of Financial Studies 30(1), 2-47.

Alfonsi, A., A. Fruth, and A. Schied (2008). Constrained portfolio liquidation in a limit order book model. Banach Center Publ 83, 9-25.

Alfonsi, A., A. Fruth, and A. Schied (2010). Optimal execution strategies in limit order books with general shape functions. Quantitative Finance 10(2), 143-157.

Alfonsi, A. and A. Schied (2010). Optimal trade execution and absence of price manipulations in limit order book models. SIAM Journal on Financial Mathematics 1(1), $490-522$.

Alfonsi, A., A. Schied, and A. Slynko (2012). Order book resilience, price manipulation, and the positive portfolio problem. SIAM Journal on Financial Mathematics $3(1)$, $511-533$.

Almgren, R. and N. Chriss (2001). Optimal execution of portfolio transactions. Journal of Risk $\underline{3}, 5-39$.

Bauso, D., H. Tembine, and T. Başar (2012). Robust mean field games with application to production of an exhaustible resource. IFAC Proceedings Volumes 45(13), 454-459.

Bauso, D., H. Tembine, and T. Başar (2016). Robust mean field games. Dynamic games and applications 6(3), 277-303.

Bayraktar, E. and M. Ludkovski (2011). Optimal trade execution in illiquid markets. Mathematical Finance 21(4), 681-701.

Bayraktar, E. and M. Ludkovski (2014). Liquidation in limit order books with controlled intensity. Mathematical Finance 24(4), 627-650. 
Bender, C., J. Zhang, et al. (2008). Time discretization and markovian iteration for coupled fbsdes. The Annals of Applied Probability 18(1), 143-177.

Bensoussan, A., J. Frehse, and P. Yam (2013). Mean Field Games and Mean Field Type Control Theory. Springer-Verlag: New York, NY.

Bensoussan, A., J. Frehse, and S. C. P. Yam (2015). The master equation in mean field theory. Journal de Mathématiques Pures et Appliquées 103(6), 1441-1474.

Bensoussan, A., K. Sung, S. C. P. Yam, and S.-P. Yung (2016). Linear-quadratic mean field games. Journal of Optimization Theory and Applications 169(2), 496-529.

Biagini, F., J.-P. Fouque, M. Frittelli, and T. Meyer-Brandis (2015). A unified approach to systemic risk measures via acceptance sets. arXiv preprint arXiv:1503.06354.

Billio, M., M. Getmansky, A. W. Lo, and L. Pelizzon (2012). Econometric measures of connectedness and systemic risk in the finance and insurance sectors. Journal of Financial Economics 104(3), 535-559.

Bo, L. and A. Capponi (2015). Systemic risk in interbanking networks. SIAM Journal on Financial Mathematics 6 6 (1), 386-424.

Brunnermeier, M. K. and L. H. Pedersen (2005). Predatory trading. The Journal of Finance 60(4), 1825-1863.

Buckdahn, R., J. Li, and J. Ma (2016). A stochastic maximum principle for general mean-field systems. Applied Mathematics \& Optimization 74(3), 507-534.

Buckdahn, R., J. Li, and J. Ma (2017). A mean-field stochastic control problem with partial observations. arXiv preprint arXiv:1702.05921.

Caines, P. E. (2014, Jun.). Mean field games. Springer Reference: Encyclopedia of Systems and Control, Eds. T. Samad and J. Ballieul., Springer-Verlag, London.

Caines, P. E. and A. C. Kizilkale (2016). E-nash equilibria for partially observed lqg mean field games with major player. IEEE Transactions on Automatic Control.

Cardaliaguet, P. (2012, Jan.). Notes on mean field games. Ceremade, France: Université Paris-Dauphine.

Cardaliaguet, P., F. Delarue, J.-M. Lasry, and P.-L. Lions (2015). The master equation and the convergence problem in mean field games. arXiv preprint arXiv:1509.02505. 
Carlin, B. I., M. S. Lobo, and S. Viswanathan (2007). Episodic liquidity crises: Cooperative and predatory trading. The Journal of Finance 62 (5), 2235-2274.

Carmona, R. and F. Delarue (2013). Probabilistic analysis of mean-field games. SIAM Journal on Control and Optimization 51(4), 2705-2734.

Carmona, R. and F. Delarue (2014). The master equation for large population equilibriums. In Stochastic Analysis and Applications 2014, pp. 77-128. Springer.

Carmona, R., F. Delarue, et al. (2015). Forward-backward stochastic differential equations and controlled mckean-vlasov dynamics. The Annals of Probability 43(5), 26472700 .

Carmona, R., F. Delarue, D. Lacker, et al. (2016). Mean field games with common noise. The Annals of Probability 44(6), 3740-3803.

Carmona, R., J.-P. Fouque, S. M. Mousavi, and L.-H. Sun (2016). Systemic risk and stochastic games with delay. arXiv preprint arXiv:1607.06373.

Carmona, R., J.-P. Fouque, and L.-H. Sun (2015). Mean field games and systemic risk: A toy model. Communications in Mathematical Sciences 13(4), 911-933.

Carmona, R. and X. Zhu (2016). A probabilistic approach to mean field games with major and minor players. Annals of Applied Probability 26(3).

Carmona, R. A. and Z. J. Yang (2011). Predatory trading: A game on volatility and liquidity. Preprint. URL: http://www.princeton. edu/rcarmona/download/fe/PredatoryTradingGameQF. pdf.

Cartea, Á., R. Donnelly, and S. Jaimungal (2014). Algorithmic trading with model uncertainty. Available at SSRN 2310645.

Cartea, A., R. F. Donnelly, and S. Jaimungal (2013, August). Algorithmic Trading with Model Uncertainty. Social Science Research Network Working Paper Series.

Cartea, Á. and S. Jaimungal (2012). Risk metrics and fine tuning of high frequency trading strategies. Forthcoming, Mathematical Finance. Available at SSRN 2010417.

Cartea, Á. and S. Jaimungal (2013). Modeling asset prices for algorithmic and high frequency trading. Applied Mathematical Finance 20(6), 512-547.

Cartea, Á. and S. Jaimungal (2016). Incorporating order-flow into optimal execution. Mathematics and Financial Economics 10(3), 339-364. 
Cartea, Á., S. Jaimungal, and J. Penalva (2015). Algorithmic and High-Frequency Trading. Mathematics, Finance and Risk. Cambridge University Press.

Cartea, A., S. Jaimungal, and J. Ricci (2014). Buy low, sell high: A high frequency trading perspective. SIAM Journal on Financial Mathematics 5 (1), 415-444.

Casgrain, P. and S. Jaimungal (2017). Trading algorithms with learning in latent alpha models. Available at SSRN eLibrary https://ssrn.com/abstract=2871403.

Cont, R. and A. De Larrard (2013). Price dynamics in a markovian limit order market. $\underline{\text { SIAM Journal on Financial Mathematics }} \underline{4}(1), 1-25$.

Delarue, F. (2002). On the existence and uniqueness of solutions to fbsdes in a nondegenerate case. Stochastic processes and their applications 99(2), 209-286.

Djehiche, B., H. Tembine, and R. Tempone (2015). A stochastic maximum principle for risk-sensitive mean-field type control. IEEE Transactions on Automatic Control 60(10), 2640-2649.

Douglas, J., J. Ma, P. Protter, et al. (1996). Numerical methods for forward-backward stochastic differential equations. The Annals of Applied Probability 6 (3), 940-968.

Epstein, L. G. (1999). A definition of uncertainty aversion. The Review of Economic Studies 66(3), 579-608.

Firoozi, D. and P. E. Caines (2016a). The execution problem in finance: A mean field game formulation. In 17th International Symposium on Dynamic Games and Applications, Urbino,Italy.

Firoozi, D. and P. E. Caines (2016b). Mean field game $\varepsilon$-nash equilibria for partially observed optimal execution problems in finance. In 2016 IEEE 55th Conference on Decision and Control (CDC), pp. 268-275.

Fouque, J.-P. and T. Ichiba (2013, Oct). Stability in a model of interbank lending. SIAM Journal on Financial Mathematics 4(1), 784-803.

Fouque, J.-P. and J. A. Langsam (2013). Handbook on systemic risk. Cambridge University Press.

Freixas, X., B. M. Parigi, and J.-C. Rochet (2000). Systemic risk, interbank relations, and liquidity provision by the central bank. Journal of money, credit and banking, 611-638. 
Garnier, J., G. Papanicolaou, and T.-W. Yang (2013). Large deviations for a mean field model of systemic risk. SIAM Journal on Financial Mathematics $\underline{4}$ (1), 151-184.

Gatheral, J., A. Schied, and A. Slynko (2012). Transient linear price impact and Fredholm integral equations. Mathematical Finance 22(3), 445-474.

Gayduk, R. and S. Nadtochiy (2016). Endogenous formation of limit order books: dynamics between trades. arXiv preprint arXiv:1605.09720.

Geering, H. P. (2007). Optimal Control with Engineering Applications. Springer Berlin Heidelberg.

Guéant, O. (2015). Existence and uniqueness result for mean field games with congestion effect on graphs. Applied Mathematics and Optimization 72(2), 291-303.

Guéant, O., J.-M. Lasry, and P.-L. Lions (2011). Mean field games and applications. Paris-Princeton Lectures on Mathematical Finance, 205-266.

Guéant, O. and C.-A. Lehalle (2013). General intensity shapes in optimal liquidation. Forthcoming in Mathematical Finance.

Guéant, O., C.-A. Lehalle, and J. Fernandez-Tapia (2012). Optimal portfolio liquidation with limit orders. SIAM Journal on Financial Mathematics $\underline{3}$ (1), 740-764.

Guilbaud, F. and H. Pham (2015). Optimal high-frequency trading in a pro rata microstructure with predictive information. Mathematical Finance 25(3), 545-575.

Haldane, A. G. and R. M. May (2011). Systemic risk in banking ecosystems. Nature 469(7330), 351-355.

Hansen, L. P. and T. J. Sargent (2007). Robustness. Princeton.

Hansen, L. P., T. J. Sargent, G. Turmuhambetova, and N. Williams (2006). Robust control and model misspecification. Journal of Economic Theory 128(1), 45-90.

Huang, J. and M. Huang (2017). Robust mean field linear-quadratic-gaussian games with unknown $L^{2}$-disturbance. arXiv preprint arXiv:1701.00196.

Huang, M. (2010a). Large-population LQG games involving a major player: the Nash certainty equivalence principle. SIAM J. Control Optim. 48(5), 3318-3353.

Huang, M. (2010b). Large-population LQG games involving a major player: the Nash

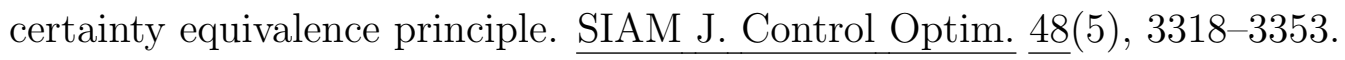


Huang, M., P. E. Caines, and R. P. Malhamé (2007, Sep.). Large-population cost-coupled LQG problems with nonuniform agents: Individual-mass behavior and decentralized $\epsilon$-Nash equilibria. IEEE Trans. Autom. Control 52(9), 1560-1571.

Huang, M., R. P. Malhamé, P. E. Caines, et al. (2006). Large population stochastic dynamic games: closed-loop mckean-vlasov systems and the nash certainty equivalence principle. Communications in Information \& Systems $\underline{6}$ (3), 221-252.

Huang, M. and S. L. Nguyen (2016). Linear-quadratic mean field teams with a major agent. In Decision and Control (CDC), 2016 IEEE 55th Conference on, pp. 6958-6963. IEEE.

Jaimungal, S. and D. Kinzebulatov (2014). Optimal execution with a price limiter. Risk, 49.

Jaimungal, S. and G. Sigloch (2012). Incorporating risk and ambiguity aversion into a hybrid model of default. Mathematical Finance 22(1), 57-81.

Karatzas, I. and S. Shreve (2014). Brownian Motion and Stochastic Calculus. Graduate Texts in Mathematics. Springer New York.

Karoui, N. and L. Mazliak (1997). Backward Stochastic Differential Equations. Chapman \& Hall/CRC Research Notes in Mathematics Series. Taylor \& Francis.

Lacker, D. and K. Ramanan (2017). Rare nash equilibria and the price of anarchy in large static games. arXiv preprint arXiv:1702.02113.

Lasry, J.-M. and P.-L. Lions (2007a). Mean field games. Japanese journal of mathematics 2(1), 229-260.

Lasry, J.-M. and P.-L. Lions (2007b). Mean field games. Jap. J. Math. 22(1), 229-260.

Lim, A. E. B. and J. G. Shanthikumar (2007). Relative Entropy, Exponential Utility, and Robust Dynamic Pricing. Operations Research 55(2), 198-214.

Maccheroni, F., M. Marinacci, and A. Rustichini (2006). Ambiguity aversion, robustness, and the variational representation of preferences. Econometrica 74(6), 1447-1498.

Maenhout, P. J. (2004). Robust portfolio rules and asset pricing. Review of Financial Studies 17(4), 951-983.

Markowitz, H. (1952). Portfolio selection. The journal of finance 7(1), 77-91. 
Moallemi, C. C., B. Park, and B. Van Roy (2012). Strategic execution in the presence of an uninformed arbitrageur. Journal of Financial Markets 15(4), 361-391.

Moon, J. and T. Başar (2017). Linear quadratic risk-sensitive and robust mean field games. IEEE Transactions on Automatic Control 62(3), 1062-1077.

Nguyen, S. L. and M. Huang (2012). Linear-quadratic-Gaussian mixed games with continuum-parametrized minor players. SIAM J. Control Optim. 50(5), 2907-2937.

Nourian, M. and P. E. Caines (2013). $\epsilon$-Nash mean field game theory for nonlinear stochastic dynamical systems with major and minor agents. SIAM J. Control Optim. 51(4), 3302-3331.

Obizhaeva, A. A. and J. Wang (2013). Optimal trading strategy and supply/demand dynamics. Journal of Financial Markets 16(1), 1-32.

Øksendal, B. and A. Sulem (2009). Applied stochastic control of jump diffusions.

Peng, S. (1990). A general stochastic maximum principle for optimal control problems. SIAM Journal on control and optimization 28(4), 966-979.

Peng, S. (1992). Stochastic hamilton-jacobi-bellman equations. SIAM Journal on Control and Optimization 30(2), 284-304.

Pham, H. (2009). Continuous-time Stochastic Control and Optimization with Financial Applications. Stochastic Modelling and Applied Probability. Springer Berlin Heidelberg.

Predoiu, S., G. Shaikhet, and S. Shreve (2011). Optimal execution in a general one-sided limit-order book. SIAM Journal on Financial Mathematics 2 (1), 183-212.

Rachev, S. T. and L. Rüschendorf (1998). Mass Transportation Problems: Volume I: Theory, Volume 1. Springer Science \& Business Media.

Rochet, J.-C. and J. Tirole (1996). Interbank lending and systemic risk. Journal of Money, credit and Banking 28(4), 733-762.

Saldi, N., T. Basar, and M. Raginsky (2017). Approximate nash equilibria in partially observed stochastic games with mean-field interactions. arXiv preprint arXiv:1705.02036.

Schied, A. and T. Zhang (2013). A state-constrained differential game arising in optimal portfolio liquidation. arXiv:1312.7360. 
Şen, N. and P. E. Caines (2014). Mean field games with partially observed major player and stochastic mean field. In Decision and Control (CDC), 2014 IEEE 53rd Annual Conference on, pp. 2709-2715. IEEE.

Skiadas, C. (2013). Smooth Ambiguity Aversion Toward Small Risks and ContinuousTime Recursive Utility. Journal of Political Economy 121(4), 775-792.

Tembine, H., Q. Zhu, and T. Basar (2011, Aug.). Risk-sensitive mean-field stochastic differential games. In Proc. 18th IFAC World Congress, Number 3222-3227, Milano, Italy.

Uppal, R. and T. Wang (2003, December). Model misspecification and underdiversification. The Journal of Finance 58(6), 2465-2486.

Yong, J. and X. Y. Zhou (1999). Stochastic controls: Hamiltonian systems and HJB equations. Springer-Verlag: New York, NY.

Zhao, W., Y. Fu, and T. Zhou (2014). New kinds of high-order multistep schemes for coupled forward backward stochastic differential equations. SIAM Journal on Scientific Computing 36(4), A1731-A1751. 